$$
\begin{gathered}
\text { WALDEN } \\
\text { UNIVERSITY } \\
\text { A higher degree. A higher purpose. }
\end{gathered}
$$

\title{
Periodicity of epidemics of invasive disease due to infection with Streptococcus pneumoniae in the United States
}

CA. Eckrode

Follow this and additional works at: http://scholarworks.waldenu.edu/hodgkinson

This Dissertation is brought to you for free and open access by the University Awards at ScholarWorks. It has been accepted for inclusion in Harold L.

Hodgkinson Award for Outstanding Dissertation by an authorized administrator of ScholarWorks. For more information, please contact ScholarWorks@waldenu.edu. 


\title{
Walden University
}

\author{
COLLEGE OF HEALTH SCIENCES
}

This is to certify that the doctoral dissertation by

\author{
Carl Eckrode
}

has been found to be complete and satisfactory in all respects, and that any and all revisions required by the review committee have been made.

Review Committee

Dr. Maria Rangel, Committee Chairperson, Public Health Faculty

Dr. Shana Morrell, Committee Member, Public Health Faculty

Dr. Hadi Danawi, Committee Member, Public Health Faculty

Chief Academic Officer

Denise DeZolt, Ph.D.

Walden University

2010 


\title{
ABSTRACT
}

Periodicity of Epidemics of Invasive Disease Due to Infection with Streptococcus pneumoniae in the United States

by

C. A. Eckrode

MPH, California College for Health Sciences, 2005

B.S., California College for Health Sciences, 2002

\author{
Dissertation Submitted in Partial Fulfillment \\ of the Requirements for the Degree of \\ Doctor of Philosophy \\ Public Health
}

Walden University

February 2010 


\begin{abstract}
Despite the availability of vaccines, every year 40,000 individuals die due to the direct effect of Invasive Pneumococcal Disease (IPD) or its complications. IPD has been associated with 100,000-135,000 hospitalizations for pneumonia, 57,000 cases of bacteremia, and 300 cases of meningitis in the United States every year. Little is known about IPD epidemic patterns beyond annual seasonality, and this lack of understanding has limited the ability to detect early changes in IPD epidemiology that may lead to large outbreaks. To mitigate this gap in understanding, a retrospective cohort design study was conducted using a population-based cohort from the National Hospital Discharge Survey for the period 1979-2006. This study set out to determine whether invasive infection by $S$. pneumoniae in the United States occurs in an epidemic pattern of a predictable recurrent nature and definable frequency. The theoretical basis for the study was drawn from the dynamic modeling of stochastic epidemic systems, and the analysis utilized time-series methods to examine the data. These analyses lead to the finding that IPD epidemics demonstrate a chaotic dynamic and a discrete, non-Markov process; that is, there is no predictable pattern to epidemics of IPD. The results of this study, that recurrent events consistent with periodic epidemics could not be identified, provide support for the current method of IPD surveillance and existing models of IPD dynamics. The present practice of mass vaccination by risk group, as opposed to vaccination for a predicted outbreak, is supported by the results of this study. These evidence-supported interventions will yield significant reductions in the morbidity and mortality associated with IPD, and the positive social change that results from improved health outcomes, reductions in suffering, and decreased health care costs.
\end{abstract}



Periodicity of Epidemics of Invasive Disease Due to Infection with Streptococcus pneumoniae in the United States

by

C. A. Eckrode

MPH, California College for Health Sciences, 2005

B.S., California College for Health Sciences, 2002

\author{
Dissertation Submitted in Partial Fulfillment \\ of the Requirements for the Degree of \\ Doctor of Philosophy \\ Public Health
}

Walden University

February 2010 
UMI Number: 3391469

All rights reserved

INFORMATION TO ALL USERS

The quality of this reproduction is dependent upon the quality of the copy submitted.

In the unlikely event that the author did not send a complete manuscript and there are missing pages, these will be noted. Also, if material had to be removed, a note will indicate the deletion.

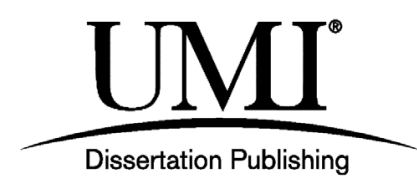

UMI 3391469

Copyright 2010 by ProQuest LLC.

All rights reserved. This edition of the work is protected against unauthorized copying under Title 17, United States Code.

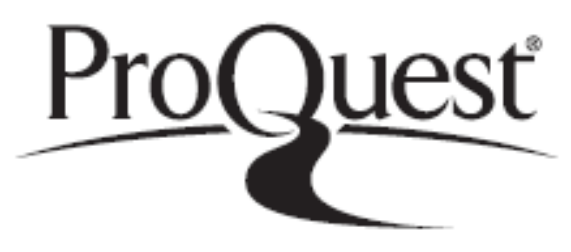

ProQuest LLC

789 East Eisenhower Parkway

P.O. Box 1346

Ann Arbor, MI 48106-1346 


\section{Dedication}

This work is dedicated to my wife, Debbie. Without her love, her patience, her support, and her inspiration this research would have not been possible.

"So oft have I invoked thee for my Muse

And found such fair assistance in my verse

As every alien pen hath got my use

And under thee their poesy disperse.

Thine eyes that taught the dumb on high to sing

And heavy ignorance aloft to fly

Have added feathers to the learned's wing

And given grace a double majesty.

Yet be most proud of that which I compile,

Whose influence is thine and born of thee:

In others' works thou dost but mend the style,

And arts with thy sweet graces graced be;

But thou art all my art and dost advance

As high as learning my rude ignorance."

William Shakespeare, Sonnet LXXVIII 


\section{Acknowledgements}

Many people have contributed to this research and this dissertation. I owe many thanks to all those who have made this research and dissertation possible.

I would like to sincerely thank Dr. Maria Rangel for her guidance, understanding, and patience during this process. Her mentorship encouraged me to grow as scholar/practitioner, and she has placed an indelible mark on my intellectual development. I am thankful for her support and the opportunity to work with such a balance of guidance and independence. I would also like to thank the other members of my committee, Dr. Shana Morrell and Dr. Hadi Danawi for their input, valuable discussions and accessibility. Their insightful comments and constructive criticisms at different stages of my research were thought provoking and they helped me focus my ideas. I am grateful to them for holding me to high standards and improving the quality of my research.

To my son, Roger, my daughter-in-law, Michelle, and my granddaughter, Melissa, who have supported this effort in a myriad of ways, my thanks and love. I would like to acknowledge my father, William Eckrode, whose insatiable curiosity about my research and his oft' expressed desire for me to finish in a prompt manner has been a source of encouragement throughout the dissertation process.

I would be remiss if I did not take this opportunity to recognize and thank my grandmother, Phyllis Helen Withycombe-Stump, Oregon State University, 1920, Physicist and Teacher. When, as a child, I asked what she did, she told me "I'm a scientist and teacher," and made it sound like the coolest thing in the world. Next to being a husband, father and grandfather, she was right.

Most importantly, none of this would have been possible without the love, patience and support of my wife, Debbie, to whom this dissertation is dedicated. She has been a constant source of strength and inspiratation. She is indispensible to this project and to my life, and words cannot adequately express my gratitude and love. 


\section{TABLE OF CONTENTS}

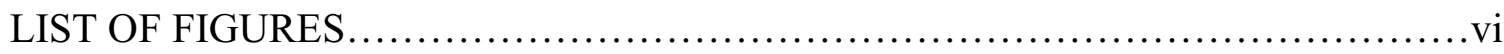

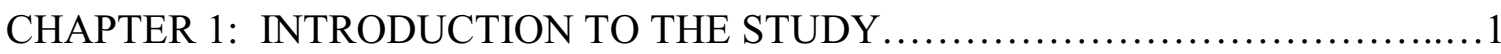

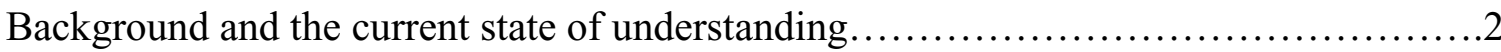

Problem Statement............................................................

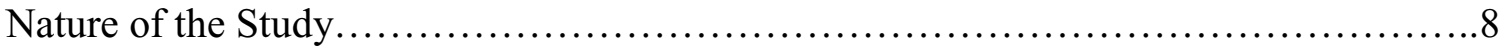

Research Questions........................................................ 8

Hypotheses..........................................................

Purpose of the Study...................................................... 10

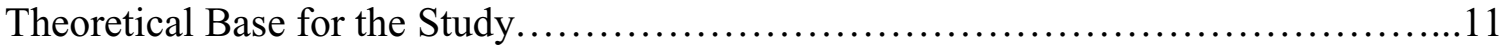

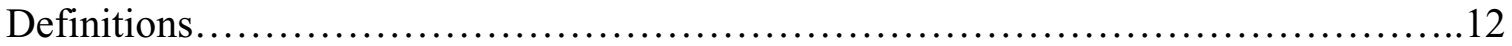

Assumptions, Limitations, Scope and Delimitations.............................. 17

Assumptions....................................................... 17

Limitations............................................................ 18

The Scope of the Study................................................ 18

Significance of the Study........................................................... 18

Filling the Existing Gap in Our Understanding of

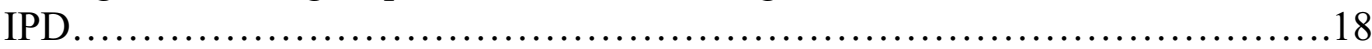

Positive Social Change Emerging from the Study ..........................20

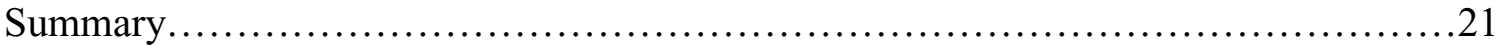

CHAPTER 2: REVIEW OF THE LITERATURE...............................23

Introduction and Background/Streptococcus pneumoniae as a Significant Human

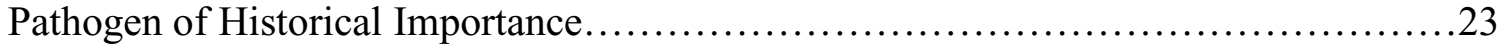

General Epidemiology....................................................... 31

Characteristics of the Causal Agent for IPD, Streptococcus pneumoniae..........31

Mode of Transmission...................................................442

Incidence and Prevalence of IPD in the United States.........................42

General Burden of IPD on the Human Population.....................42

Incidence of Invasive Pneumococcal Infections in the United States.............................................44

Streptococcus pneumoniae Colonization and Infection, and Risk Factors for IPD...........................................................48

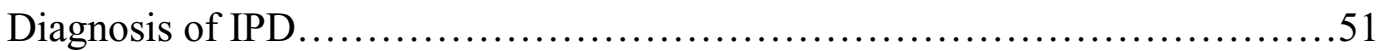

Antibiotics in the Treatment of IPD .......................................52

The Emergence and Impact of Drug-Resistant Streptococcus pneumoniae (DRSP)............................................... 54 


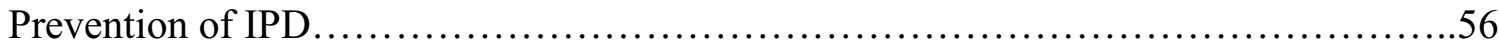

Adult Pneumococcal Vaccines..............................................56

Pediatric Pneumococcal Vaccines........................................5 57

Temporality and Seasonality of Infectious Diseases.............................. 59

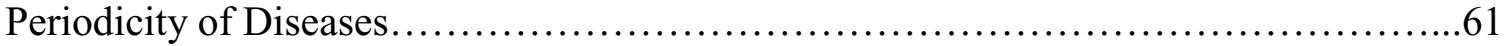

Models based on Time-Series Analysis..........................................64

Deterministic Models...................................................66

Stochastic Models.....................................................67

Modeling of Disease Dynamics Using Time-Series Approaches................69

Previous Attempts at Constructing a Model of

Sepsis Epidemic Patterns...................................................71

A Model of IPD Dynamics Amenable to Modification as a

Temporally-Forced Model using Time-Series Analysis.......................72

A Recommended Approach for Description of

Epidemic Patterns of IPD/ Stochastic Approaches............................75

National Hospital Discharge Survey (NHDS) as a Secondary Data Source for

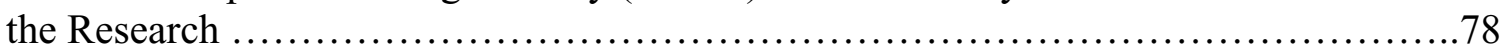

Definition and History of the NHDS ..................................... 78

NHDS Data Sources and Contributors....................................80

The Structure of NHDS Data............................................. 81

The Use of ICD-9CM Coding as a Key Variable in the NHDS, and

Quality Assurance for Coding and Data Accuracy...........................82

Correlation of NHDS Data to Population Estimates is

Essential to Calculating Incidence............................................8 83

Privacy Protections in the NHDS ........................................ 84

Strengths and Limitations of the NHDS..................................84

A Serious Gap in Our Knowledge of IPD Epidemic Dynamics...................... 87

CHAPTER 3: METHODS ....................................................... 89

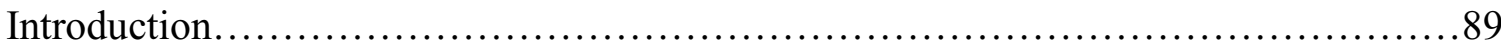

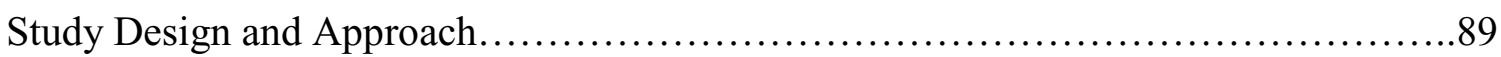

Setting and Sample............................................................. 92

Description of the Study Population.................................... 94

Case Definition.................................................... 94

Operational Definition of IPD ......................................... 95

Sampling Method and Frame........................................... 95

Eligibility Criteria.................................................... 97

Instrumentation and Materials....................................................98

Study Instruments, Type of Instrument, and Concepts Measured

by the Instruments................................................98

A Detailed Description of the Variables Dontained in the Data.................99 
Processes for Assessment of the Reliability and Validity of

the Instruments.......................................................... 102

Location of the Raw Data.............................................. 104

Data Collection and Analysis................................................. 105

Measures for the Protection of Participant Rights................................110

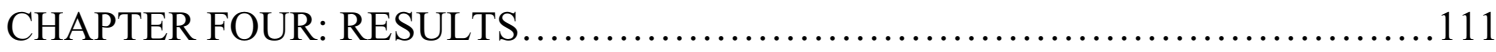

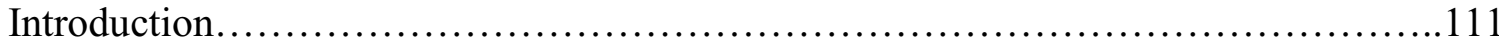

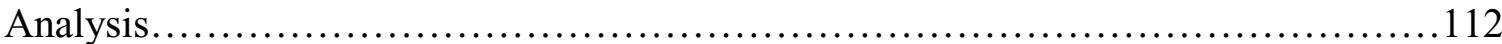

IPD Incidence in the United States......................................112

IPD Endemic Levels in the United States................................... 114

IPD Seasonality in the United States..................................... 114

IPD Periodicity in the United States.................................... 115

Results....................................................................115

IPD Incidence in the United States........................................ 115

Strafication by Age Group, Gender, and Region............................116

IPD Endemic Levels in the United States................................ 118

Periodicity of IPD Epidemics in the United States...........................118

IPD Periodicity Stratified by Gender, Geographic region, and Age Group.......119

Summary ................................................................. 124

CHAPTER FIVE: DISCUSSION

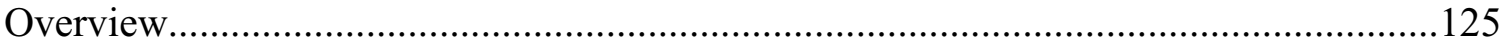

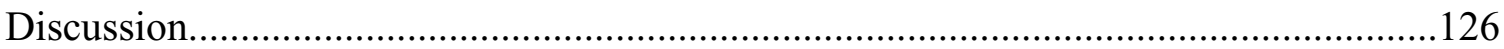

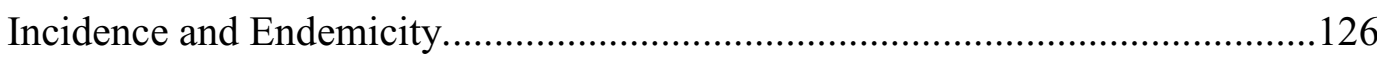

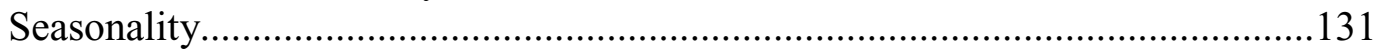

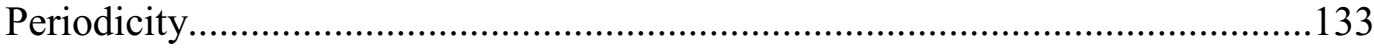

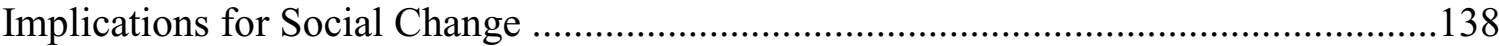

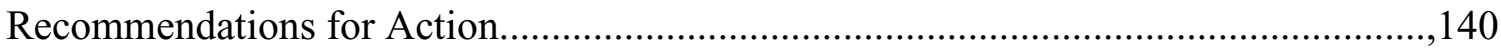

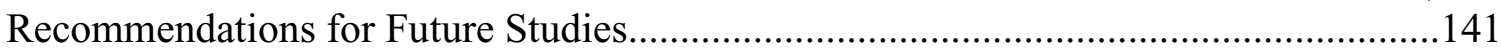

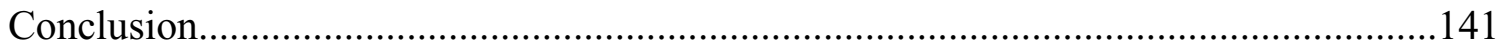

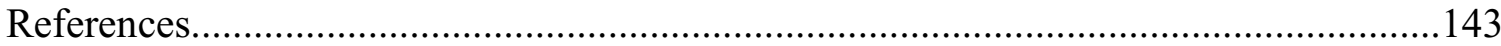

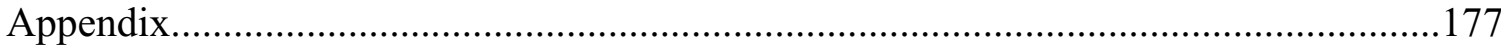

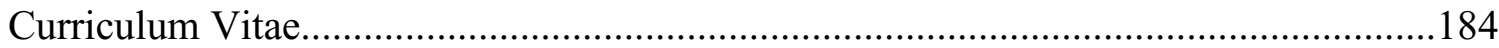




\section{LIST OF FIGURES}

Figure 1. Incidence of IPD in the United States, 1979-2006, per 100000....................116

Figure 2. Incidence of IPD in the United States, 1979-2006, by age...........................117

Figure 3. Incidence of IPD in the United States, 1979-2006, by gender........................117

Figure 4. Incidence of IPD in the United States, 1979-2006, by geographic region.......118 Figure 5. Periodogram of IPD incidence in the United States for the period from 19792006, inclusive of all ages, genders, and geographic regions

Figure 6. Periodogram of IPD incidence in the United States for the period from 19792006, by gender, male.

Figure 7. Periodogram of IPD incidence in the United States for the period from 1979-

2006, by gender, female.

Figure 8. Periodogram of IPD incidence in the United States for the period from 1979-

2006, Northeast, Midwest, South, and West census regions.

Figure 9. Periodogram of IPD incidence in the United States for the period from 19792006, 0-4 years of age, 55-64 years of age, 65-74 years of age,

75 years of age and older.

Figure 10 . Periodogram of IPD incidence in the United States for the period from 1979-

2006, analyzed for seasonality of outbreaks.

Figure 11. Periodogram of IPD incidence in the United States for the period from 1979-

2006, 5-14 years of age 182

Figure 12. Periodogram of IPD incidence in the United States for the period from 19792006, 15-24 years of age. 182

Figure 13. Periodogram of IPD incidence in the United States for the period from 19792006, 25-34 years of age. 183

Figure 14. Periodogram of IPD incidence in the United States for the period from 1979-

2006, 35-44 years years of age.

Figure 15. Periodogram of IPD incidence in the United States for the period from 19792006, 45-54 years years of age. 


\section{CHAPTER 1: INTRODUCTION TO THE STUDY}

Background

Invasive pneumococcal disease (IPD), resulting from invasion of a normally sterile site by the gram-positive bacterium Streptococcus pneumoniae (S. pneumoniae) (Centers for Disease Control and Prevention, 2007), is responsible for morbidity, deaths, and as a result, use of healthcare resources (Ament, Baltussen, Duru, Rigaud-Bully, de Graeve, \& Christie et al., 2000; Balakrishnan, Crook, Morris, \& Gillespie, 2000; Poland, 1999; Whitney, Farley, Hadler, Harrison, Lexau, \& Reingold et al., 2000). Over a century after identification of S. pneumoniae by Pasteur and Sternberg (Austrian, 2000; BridyPappas, Margolis, Center, \& Isaacman, 2005; Gray, 2000; Pasteur, 1881; Sternberg, 1881), a sizeable body of knowledge has been accumulated, covering the characteristics

of the bacterium (Austrian, 2004), its interaction with its human hosts (Austrian, 2004), and the treatment of diseases resulting from infection with S. pneumoniae (Austrian, 2004). However, much remains unknown about the epidemic pattern of IPD. This is a serious deficiency, inhibiting our ability to predict outbreaks of this set of serious and potentially life-threatening diseases. The following overview covers the current state of understanding of IPD, its causal factors, the seasonality of IPD, and an approach to understanding the overall epidemic pattern of IPD. A brief examination of the technique used in determining the existence of an epidemic pattern is also provided. A more thorough review is found in chapter 2. 
The Current State of Understanding

IPD is responsible for approximately 40,000 deaths due to complications of infections occurring annually in the U.S. (Bloom, Wheeler, \& Lynn, 1999; McDaniel \& Swialto, 2004). The impact of IPD on morbidity is also high, with as many as $100,000-$ 135,000 hospitalizations for pneumonia, 57,000 cases of bacteremia, and 300 cases of meningitis annually in the United States (CDC, 2006). IPD affects the very young, the old, the immnunocompromised, and indigenous groups more than the general population (Butler, 2004). Children (McNabb et al., 2007), African Americans (Robinson et al., 2001), Alaska Natives (Butler \& Schuchat, 1999), and Native Americans (Benin et al., 2003) are among the groups with highest risk for the disease.

The following section reviews the mechanisms employed by $S$. pneumoniae to survive the host immune response and the mechanisms by which it achieves sufficient levels of colony survival to remain endemic in the population. These survival mechanisms include the ability of $S$. pneumoniae to alter the host/pathogen relationship, serotype variations, and the acquisition of drug resistance (Bridy-Pappas et al., 2005). Variability in preventive measures affects infection dynamics, which over time is a major determinant of epidemic behavior (Grenfell et al., 2004). It is IPD epidemic behavior that this study sought to understand.

S. pneumoniae is a human-restricted commensal that will be acquired at one point or another by the majority of the population (Sleeman et al., 2006; Bridy-Pappas et al., 2005). Nearly all children experience bacterial colonization and carriage during the first two years of life (Brueggemann, Peto, Crook, Butler, \& Kristinsson, 2004; Hammitt, Bruden, Butler, Baggett, Hurlburt, Reasonover et al., 2006). The pediatric subpopulation 
serves as a reservoir for transmission to other subpopulations. Adults who live with a child under five years of age, or a child who has not received appropriate vaccination, are more likely to be colonized than adults who do not live with children under five years of age (Hammitt et al., 2006). Colonization with $S$. pneumoniae is often a normal, benign, asymptomatic condition. However, when $S$. pneumoniae invades the sterile tissues of the body, life-threatening disease may result (Pericone, Park, Imlay, \& Weiser, 2003). Invasiveness is not required for bacterial life or reproduction (Briles, Novak, Hotomi, Ginkel, \& King, 2005). However, the ability of virulent strains of $S$. pneumoniae to evade host defenses confers some advantage over less virulent strains within the same host. The selection pressures within the individual host might encourage the growth of any bacterium that successfully eludes host immune responses. Thus, the more virulent strain exchanges transmissibility from the host for the ability to reproduce (Lipsitch \& Moxon, 1997). Whether as a result of host selection pressure or chance, the pneumococcus might leave the nasopharynx, invade sterile tissues, and cause disease in some vulnerable hosts. The mechanism for this action is dependent on bacterial morphology, and variation in morphology provides the biologic mechanism needed for development of IPD in the susceptible host (Rytel, Dee, Ferstenfeld \& Hensley, 1974). Infection, however, is distributed in the population according to risk factors. As S. pneumoniae relies on airborne transmission, contact patterns between colonized and susceptible persons determine transmission patterns, and can provide selection pressures that force bacterial mutation, further promoting virulence and invasiveness (Petermann \& Rios, 2004; Temime, Boelle, Opatowski, \& Guillemot, 2008; Wallinga, Edmunds, \& Kretzschmar, 1999). 
Prior to the availability of effective antibiotics and vaccines, epidemics of pneumococcal disease were common in closed populations, such as prisons, military barracks, and dormitories (Butler \& Cetron, 1999; Hoge, Reichler, Dominguez, Bremer, Mastro, Hendricks et al., 1994). Outbreaks within these closed communities are less common today, but still occur, often in nursing homes and other residential health care settings (Romney et al., 2008). Long-term care facilities also provide a reservoir for $S$. pneumoniae (Butler \& Cetron). In the United States and several other countries, day care centers have been identified as environments favorable for selection and amplification of S. pneumoniae, including drug-resistant strains (Dagan et al., 2002; Lencastre \& Tomasz, 2002).

Invasive pneumococcal infections have a bimodal age distribution, most often affecting children younger than five years and adults older than 65 years. However, IPD can occur in any age group and is associated with a high mortality rate, exhibiting in some cases as much as a $20 \%$ case-fatality rate (Christenson, Lundbergh, Hedlund, \& Ortqvist, 2001; McDaniel \& Swialto, 2004). The incidence of IPD varies across the US, with different communities displaying widely different rates (Huang, Finkelstein, \& Lipstich, 2005). This variation in rate may be due to differences in contact patterns (Bansal, Grenfell, \& Meyers, 2007) and these differences prevent complete eradication of S. pneumoniae colonization, as between patch transmission prevents local extinctions from becoming global extinctions (Hagenaars, Donnelly, \& Ferguson, 2004).

Considering host factors in addition to age, it has been shown that $S$. pneumoniae presents a risk of disease to several distinct groups (Robinson et al., 2001; Butler \& Schuchat, 1999). Susceptible populations include those with conditions that significantly 
increase a patient's vulnerability to infectious disease, such as cardiovascular disease, cardiac transplant, cerebrovascular disease, lower and chronic respiratory diseases, renal disease, diabetes mellitus, chronic alcoholism and its complications, sickle cell disease, asplenia (either functional or anatomic), HIV infection, genetic anomalies of the immune system, and malignant neoplasms (Amber, Gilbert, Schiffman, \& Jacobson, 1990; Gilbert et al., 2002; Haddad et al., 2008; Hjuler et al., 2007; Juhn et al., 2008; Smith, Erlandson, Shulman, \& Stern, 1957; Torres \& Bisno, 1973). Epidemics often display temporal variability, with variation in the causal agent, host responses, and transmission affecting this variability (Bansal et al., 2007). Seasonal changes in disease incidence, that is, cyclic and predictable changes in disease patterns varying by the seasons of the year, have been identified for many diseases (Bauch \& Earn, 2003). IPD has a seasonal pattern of incidence (Dowell, Whitney, Wright, Rose, \& Schuccat, 2003). It is known that $S$. pneumoniae outbreaks occur during winter months (Dowell, Whitney, Wright, Rose, \& Schuccat, 2003; Kim et al., 1996: Talbot et al., 2005), with the exception of Alaska (Davidson et al., 1989). The reason behind this seasonality remains unclear, with suggestions ranging from temperature influences to seasonal changes in host physiology (Dowell et al., 2003; Dowell, 2001). Whatever the cause, seasonality might be viewed as a component of another larger phenomena known as periodicity, the frequency-dependent recurrence of epidemics (Yulemetyev et al., 2003).

Periodic epidemic patterns, that is, patterns of epidemics at a larger time-scale than seasonal, have been identified and examined for other endemic infectious diseases, and this knowledge used for the prevention of these diseases. Temporal models of disease dynamics provide many of the tools needed to identify periodic epidemic patterns. Using 
a temporal model applied to epidemic phenomenon, Finkenstaedt and Grenfell (2000) showed changes in the epidemic pattern of measles. Additionally, Broutin, Guegan, Elguero, Simondon and Cazalles (2005), and Guris et al. (1999) used this model to establish the quadrennial pattern of pertussis outbreaks. The identification of a pertussis epidemic outside of this expected frequency led to identification of waning immunity in the adult population (Broutin, Guégan, Elguero, Simondon, \& Cazelles, 2005; Galanas et al., 2006; Güris et al., 1999). On the other hand, influenza, once thought to show an epidemic pattern of ten year intervals, has been shown to be aperiodic through the application of temporal modeling (Dowdle, 2006); this result disallowed the use of relative frequency for influenza epidemic planning, forcing epidemiologists and public health planners to rely on other techniques in order to project influenza epidemics (Viboud, Boelle, Carrat, Valleron, \& Flahault, 2003). Establishing or refuting the existence of a recurrent epidemic pattern for IPD will allow public health practitioners to apply similar techniques, with similar results.

It is not known whether IPD epidemics occur in a periodic fashion, outside of our understanding of the seasonality of the diseases. Currently, there is limited understanding of IPD epidemic patterns beyond annual seasonality. Dowell (2001) has acknowledged the absence of this research, reasoning that annual seasonal patterns can be more easily "locked in" than can long-term patterns. A study that typifies the work done on the annual seasonality of IPD was conducted by Dowell, Whitney, Wright, Rose, and Schuchatt (2003). These researchers used information from the Active Bacterial Core Surveillance database and an unspecified time-series analysis process to clearly establish the annual seasonality of IPD. However, this study did not examine the epidemic pattern 
outside of the annual seasonality. Other studies examining the epidemic pattern of IPD have concentrated on examining the relationship between annual seasonality and other respiratory infections (Ampofo et al., 2008; Butler \& Schuchat, 1999; Kim et al., 1996; Talbot et al., 2005), but not the epidemic pattern beyond a year. A study that might have revealed the epidemic pattern of a subtype of IPD, pneumococcal bacteremia, was prevented from doing so by the inclusion of other causes of bacteremia in the sample population model (Danai, Sinha, Moss, Haber, \& Martin, 2007). The study reported increased incidence of bacteremia during a time period when other studies reported decreased IPD incidence (Hammitt et al., 2006; Jackson \& Janoff, 2008; McBean, Park, Caldwell, \& Yu, 2005; Moore et al., 2004; Poehling et al., 2006; Shah \& Ratner, 2005). This could have been due to the influence of the epidemic pattern of pathogens other than S. pneumoniae, and precludes use of the results in understanding IPD epidemic patterns.

Several characteristics of IPD and the biology of $S$. pneumoniae provide a plausible basis for a periodic epidemic pattern to exist. However, no study analyzing IPD incidence rates over time in the United States (or any other location) to establish periodicity could be found in a thorough review of the existing literature. This study proposes to address this gap.

\section{Problem Statement}

There is a substantial body of evidence that establishes $S$. pneumoniae as a significant human pathogen, and IPD as an important cause of morbidity and mortality (Bloom, Wheeler, \& Lynn, 1999; Centers for Disease Control and Prevention, 2006; Dochez \& Avery, 1915; McDaniel and Swialto, 2004; Osler, 1898). Currently, the annual seasonality is understood, but not the larger epidemic patterns of IPD, and we lack 
information needed to plan, execute, and evaluate preventive measures, and to identify events where the pathogen has changed its epidemiology (Broutin, Guégan, Elguero, Simondon, \& Cazelles, 2005; Galanas et al., 2006; Güris et al., 1999). Therefore, further examination of the IPD epidemiology with an emphasis on the disease epidemic characteristics over time is needed to help guide prevention strategies. This investigation provides knowledge that helps to addresses this significant issue of public health.

Nature of the Study

In order to describe the epidemic pattern, a retrospective cohort study was performed. Examining a cohort taken from the National Hospital Discharge Survey database for the time period 1979 to the present resulted in a detailed retrospective description of the large epidemic pattern of invasive disease caused by $S$. pneumoniae. The study set out to establish the existence or absence of a recurrent epidemic pattern and frequency, with the intent of enhancing our understanding of IPD. The study determined the incidence of IPD during seasonal increases and established the endemic level of the disease in the United States from 1979-2006 to answer the following research question regarding the patterns of IPD incidence over time, in all ages, genders, and geographic locations, in the United States:

Research Questions

The study answered the following research questions:

(1) Do epidemics of IPD occur in a periodic manner with a definable frequency in addition to the predicted seasonal increase in incidence, in the aggregate, in the United States? 
(2) Do epidemics of IPD occur in a periodic manner with a definable frequency in addition to the predicted seasonal increase in incidence, by gender, in the United States?

(3) Do epidemics of IPD occur in a periodic manner with a definable frequency in addition to the predicted seasonal increase in incidence, by age (in ranges), in the United States? And

(4) Do epidemics of IPD occur in a periodic manner with a definable frequency in addition to the predicted seasonal increase in incidence, by geographic area, in the United States?

\section{Hypotheses}

The study answered the research questions by testing the following null and alternative hypotheses:

(1) $\mathrm{H}_{\mathrm{o}}$ : IPD did not occur in periodic epidemics of a definable frequency outside of the predicted seasonal increase in incidence in the United States for the period from 1979-2006, in the aggregate.

(2) $\quad \mathrm{H}_{\mathrm{a} 1}$ : IPD occurred in periodic epidemics of a definable frequency in addition to the predicted seasonal increase in incidence in the United States during the period from 1979-2006, in the aggregate. $\mathrm{H}_{\mathrm{o} 2}$ : IPD did not occur in periodic epidemics of a definable frequency outside of the predicted seasonal increase in incidence in the United States for the period from 1979-2006, by gender. 
$\mathrm{H}_{\mathrm{a} 2}$ : IPD occurred in periodic epidemics of a definable frequency in addition to the predicted seasonal increase in incidence in the United States during the period from 1979-2006, by gender. $\mathrm{H}_{03}$ : IPD did not occur in periodic epidemics of a definable frequency outside of the predicted seasonal increase in incidence in the United States for the period from 1979-2006, by age (in ranges). $\mathrm{H}_{\mathrm{a} 3}$ : IPD occurred in periodic epidemics of a definable frequency in addition to the predicted seasonal increase in incidence in the United States during the period from 1979-2006, by age (in ranges). $\mathrm{H}_{\mathrm{o} 4}$ : IPD did not occur in periodic epidemics of a definable frequency outside of the predicted seasonal increase in incidence in the United States for the period from 1979-2006, by geographic area. $\mathrm{H}_{\mathrm{a} 4}$ : IPD occurred in periodic epidemics of a definable frequency in addition to the predicted seasonal increase in incidence in the United States during the period from 1979-2006, by geographic area.

A more detailed discussion of the objectives, research question and hypotheses will take place in chapter 3.

\section{Purpose of the Study}

The purpose of this study was to determine whether IPD epidemics in the United States occur in a periodic fashion, with a predictable frequency. Two objectives were addressed in order to answer that question. First, what was the endemic level of IPD in the United States for the period from 1979-2006 in the aggregate, and by gender, age (in ranges), and geographic area? Second, what was the incidence level of IPD during 
seasonal increases in the period from 1979-2006? The answers to these questions provided information fundamental to the purpose of the study.

Theoretical Base for the Study

The identification of epidemic patterns, and construction of a corresponding epidemic model, is based upon a sound theoretical construct drawn from the concept of dynamic modeling of epidemics (Keeling \& Rohani, 2008a). Specifically, the temporally forced model by Keeling and Rohani (2008c) provides the foundation for the study. This model has an extensive history of use in the examination of epidemic patterns in varicella, measles, pertussis, mumps, and rubella (Keeling \& Rohani) as discussed in chapter 2 .

One of the strengths of the temporally forced model is that it can be applied to random systems (Keeling \& Rohani, 2008c). In a random system, where the disease is endemic, the influence of external events (such as a new pool of susceptible subjects) on the normal random state can adjust the chaotic and random behavior towards regularity (Yulmetyev, Yulmetyeva, \& Gafarov, 2005). Application of dynamic modeling methods and time series analysis to epidemic disease permits the quantification of the degree of randomness, or conversely, the regularity and predictability of a system (Yulmetyev et al., 2003). Should regularity and predictability exist in an epidemic disease, it then becomes possible to use this knowledge to develop a predictive model (Yulmetyev et al., 2005). The study could have used the results to modify an existing Temporally Forced Model of IPD dynamics (Sutton, Banks \& Castillo-Chavez, 2008). This model would have been modified by applying any periodic component as a factor in the existing models' transfer rate equation to reflect a cyclical increase or decrease in disease 
incidence at the dominant frequency. As no periodicity was identified, the existing model stands. This will be addressed further in the review of the literature in chapter 2. The following section provides definitions of uncommon or otherwise ambiguous terms that will be used throughout subsequent chapters.

\section{Definitions}

23-Valent Pneumococcal Polysaccharide Vaccine (23PPV) (Pneumovax or PnuImmune: A purified polysaccharide vaccine targeted against 23 different serotypes of the Streptococcus pneumoniae bacterium (American Public Health Association, 2000).

Chaos: The result of stochastic behavior occurring in and influencing a deterministic system (Stewart, 2002). For example, when an erratic time series is observed within a deterministic system, the system is exhibiting chaos (Taniguchi \& Kakizawa, 2000).

Clockwork Process: A term describing a system dynamic that appears to occur as if by the mechanism of a gear-driven machine, with no variation or deviation from the predicted path (Bjornstad, Finkenstadt, \& Grenfell, 2002).

Colonization: The establishment of a microbial colony with no signs of disease (Hierholzer, 1996).

Cooley-Tukey Algorithm: A Fast Fourier Transform (FFT) algorithm for use in computer processing. By the use of recursive operations (in this context, dividing the main problem into sub-problems of the same type), the Cooley-Tukey implementation of the FFT reduces a large Discrete Fourier Transform into several smaller Discrete Fourier Transforms. This reduces the time and complexity of a Fourier Transform, and facilitates 
the design and use of computer programs for Fourier Transforms (Cooley \& Tukey, 1965).

Deterministic System: A system in which randomness does not influence the development of future states of the system; the same starting conditions will always result in the same observed output (Aron, 2007; Keeling and Rohani, 2008d).

Deterministic Model: A model where, given the same starting conditions (initial conditions) the exact same pattern or trajectory is observed for each realization of the model (Keeling \& Rohani, 2008b).

Disease Fadeout: The extinction of a disease expressed in a mathematical model, most likely as a result of a sufficient drop in the number of infected and susceptible individuals in a given population (Keeling \& Rohani, 2008b).

Endemic Level: The usual or expected level of disease in a defined population during a defined period of time (Hierholzer, 1996).

Epidemic: An unusual and higher than expected level of disease in a defined population in a defined period of time (Hierholzer, 1996).

Fourier Transform: The mathematic construction of an arbitrary function using sinusoids, so that the functions being studied can be written as linear combinations. Any periodic function can be represented as a series of harmonically related sinusoids, and the application of the mathematic construction of arbitrary functions using these sinusoids is called Fourier analysis (Wei, 1994b; Ziemer, Tranter, \& Fannin, 1983).

Fast Fourier Transform (FFT): The FFT is a means for efficiently computing the discrete Fourier transform for frequency domain time-series analysis. It overcomes the 
computational complexity of an $n$-point discrete Fourier transform through an iterative process by means of trigonometric and complex exponential functions (Wei, 1994b).

Frequency Domain Analysis: A statistical method for describing a fluctuating time series in terms of sinusoidal behavior at a set of frequencies. The fundamental approach to frequency domain analysis is the Fourier transform, which decomposes a periodic function into a sinusoidal function (Wei, 1994b).

Herd Immunity: The resistance of a population to invasion by and spread of an infectious disease due to sufficient levels of immunity in that population (Fine, 1993). Index Case; The first recognized case in a series of cases in a given host population (Hierholzer, 1996).

Infectivity: The ability of a pathogen to cause infection in a susceptible host (Nelson, 2007).

Invasive Pneumococcal Disease (IPD):_The disease resulting from invasion of a normally sterile site by the bacterium Streptococcus pneumoniae (CDC, 2007). Therefore, the presence of $S$. pneumoniae in the blood or neural tissues, including CSF, meets the conceptual definition for this study.

ICD-9-CM. The International Classification of Diseases $9^{\text {th }}$ Revision, Clinical Modification. A scheme for classification of diseases, where diagnoses are coded into three to six digit numbers (Kongstvedt, 2003).

Markov Process: A stochastic process where future probabilities are determined by the most recent conditions, not by past events (Gnedenko, 1962; Kemeny \& Snell, 1960; Papoulis, 1984). 
Markov Chain: A type of Markov Process; in a Markov Chain, only the current state has any influence on the future of the system, and then only on the next state. Therefore, future states are based in probability, rather than deterministically (Kemeny \& Snell, 1960).

Noise: For the purposes of this study, noise refers to the uncertainty in the recorded data that can influence the degree of stochasticity in the epidemic dynamics. This noise can arise from under- or over-reporting of the disease or misdiagnosis (external noise) (Keeling \& Rohani, 2008b).

Non-Vaccine Types: S. pneumoniae serotypes not targeted by either one or both of the approved pneumococcal vaccines (Hausdorff, Bryant, Kloek, Paradiso \& Siber, 2000).

Periodicity: In terms of epidemic dynamics, periodicity is defined as the frequency-dependent recurrence of epidemics; a larger phenomena than seasonality (Yulmetyev et al., 2003).

Periodogram: A periodogram is the visual representation of the periodic components in a series of observations resulting from a Time Series Analysis by the Fourier or Fast Fourier Transform approaches (Wei, 1994a).

PnCRM7 (Prevnar): The heptavalent conjugated vaccine currently approved and recommended for pediatric administration in the United States (Bernatoniene \& Finn, 2005). This vaccine formulation includes seven of the most common invasive serotypes of S. pneumoniae. This vaccine does not confer lifetime immunity to IPD (Millar et al., 2006). 
Seasonality: Cyclic changes in disease patterns varying with annual recurring events, such as weather patterns and school starts (Fisman, 2007; Keeling \& Rohani, 2008c).

Sinusoidal Behavior: The results of a mathematic transform of information to the form of sine waves (sinusoids). The sinusoids, when expressed graphically in the form of periodograms, can be used to identify periodic signals or events, and to determine their frequency (Ziemer, Tranter, \& Fannin, 1983).

Stationary Process: A stationary process refers to any stochastic process that increases or decreases systematically over time (Wei, 1994c).

Stochasticity: The presence of time indexed random variables in a process; e.g. random behavior in a system over time (Wei, 1994c). Because of the chance nature of disease transmission, stochasticity is inherent in epidemic processes (Keeling \& Rohani, 2008a).

Stochastic Model: A mathematic model that approximates and accounts for random events occurring in time and addresses any Markov property present in the dynamics of an epidemic. For most population sizes, a stochastic model will be more realistic than a deterministic model, but will be more complex mathematically (Keeling \& Rohani, 2008a).

Time Series: A sequence of data points taken at specific time intervals. These data points are considered to be dependant or correlated, and thus the order of the observations is important (Wei, 1994a). 
Time-Series Analysis: A statistical method for analysis of a time series or other ordered sequences of observations; particularly in terms of analysis and prediction made of observations taken on defined and often evenly spaced time intervals (Wei, 1994e).

Virulence: The relative intrinsic ability of an agent to produce disease, or the severity of the disease and damage to the host after infection by an agent (Hierholzer, 1996; Nelson, 2007).

Assumptions, Limitations, Scope and Delimitations

Assumptions

A key assumption of this study was the accuracy of the data from the NHDS database. The reliance of the NHDS on ICD-9CM codes for classifying discharge diagnosis means that the quality of the data set is greatly dependent on the accuracy of coding (Guevara et al., 1999). To ensure accurate data, the NHDS employs quality control techniques dependant on the submitted format. For the most part, coding errors are the greatest hazard to reliability of the data within the NHDS (Popovic, 2001), and error detection procedures are in place to ensure that these hazards are reduced. Hospital discharge data have been considered by some to be more accurate and reliable than that obtained by surveys, as the information on diagnosis is obtained directly from the health care providers. Recall bias on the part of the patient is thus less of a concern (Machlin, Cohen $\&$ Thorpe, 2000). Therefore, the accuracy of the NHDS data may be assumed. Additional discussion of the NHDS, its sampling frames and techniques, and studies addressing the accuracy of the NHDS are included in chapter 2. 


\section{Limitations}

A key limitation to the study related to analysis of the data by race or ethnicity. Data on race and ethnicity within the NHDS is often missing (Dennison \& Pokras, 2000), or underreported (Kozak, 1995). This missing data may be attributed to the fact that many hospitals are not mandated to gather this information. As of 2005, only 22 states required hospital collection of this information, and often, the information was not always collected (Schoenman et al., 2005). Thus, the research excludes the use of race or ethnicity in analysis.

The Scope of the Study

The study examined the periodicity of IPD within the United States only. The occurrence of IPD outside of this defined geographical area was not considered in the analysis. Thus, generalization to populations outside of the United States, where vaccine uptake and prevalent $S$. pneumoniae serotypes may differ, may not be valid.

Additionally, the study was bounded by the characteristics of the NHDS. Occurrence of IPD in hospitals that do not report discharges to the NHDS will not factor into the analysis. Although this boundary to the study may have impacted on the calculation of incidence rates, it should not preclude the determination of any pattern of IPD epidemics, as the NHDS provides a sufficiently large population to detect perturbations in the epidemic dynamics.

Significance of the Study

Filling the Existing Gap in Our Understanding of IPD

The pattern, if any, of pneumococcal outbreaks had yet to be established. It is important in light of $S$. pneumoniae's endemicity and potential lethality that a lack of 
understanding of the IPD dynamic did not continue. It is this gap in our knowledge that this study will help to fill.

Currently, there is a dearth of research on large epidemic patterns in $S$. pneumoniae invasive infections; thus, public health and clinical practitioners lack the fundamental knowledge to make the appropriate choice of predictive models for longterm planning, and epidemics may go unacknowledged or their severity unrecognized when they deviate from the normal pattern or rate of prevalence. Furthermore, identification of epidemic patterns can aid in the assessment of vaccine efficacy; that is, the extent to which the current vaccines address the most common virulent serotypes. If an epidemic pattern changes, or if an epidemic pattern emerges, this change in disease dynamics might alert practitioners to a change in IPD epidemiology that requires attention and action. As an example of changing IPD epidemiology, there are reports of pneumococcal serotypes responsible for IPD that are not addressed by either of the current pediatric or adult vaccines. There are several of these non-vaccine types (NVTs) associated with IPD in the post-vaccine era, and at least one report refers to a fluctuating pattern in NVT-associated IPD incidence (Akduman, Ehret, \& Judson, 2006), reflecting the impact of NVT emergence on epidemic dynamics. The knowledge that was gained as a result of this study has a potential impact on the planning and execution of public health interventions to prevent or ameliorate outbreaks of IPD, and the potential for positive social change through the reduction of death and disability. 
Positive Social Change Emerging from the Study

The results of this study will facilitate improved disease surveillance through the use of robust mathematical models to predict epidemics. The improvement in surveillance emerges from the ability to identify outbreaks occurring at variance with the predicted pattern. If a novel serotype, or a serotype not addressed by the vaccines, emerged as a significant pathogen, or if vaccine-mediated immunity waned earlier than predicted, the periodic pattern of IPD epidemics (if a pattern exists) might vary, alerting practitioners to the need for further investigation. Additionally, this information might lead to enhanced disease surveillance through the ability to employ long-run probability calculation (Kachigan, 1982) for epidemic forecasting.

This improved surveillance and predictive capability could significantly reduce the public health impact of IPD by reducing the number of hospitalizations, and providing an impetus for vaccination. This promotes positive social change through improved health outcomes and decreased health care costs (Ament et al., 2000). Currently, prevention interventions targeted against IPD emphasize vaccination, particularly in the pediatric population (Overturf, 2000; Parsons \& Dockrell, 2002). Although vaccination has been successful in reducing the incidence of IPD, vaccination against $S$. pneumoniae is not universal, as some people elect to not vaccinate their children or themselves. Establishment of a pattern to IPD epidemics could facilitate prediction of outbreaks; vaccination campaigns can then target these unvaccinated subpopulations prior to the periodic rise in incidence and encourage them to receive vaccination. Unvaccinated individuals might well seek vaccination in the face of a 
predicted epidemic, rather than relying on herd immunity (Bauch, 2005), thus decreasing morbidity and mortality.

The efficacy of pneumococcal vaccines, and vaccine coverage of the most common virulent serotypes is an issue of some importance (Jackson et al., 2003; Madhi, Whitney, \& Nohynek; Maki, 2004; McDaniel \& Swialto, 2004) and on-going research (e.g., Maekelae \& Kayhty, 2004; Whitney et al., 2006). Assessment of vaccine efficacy and changes that might be required in vaccine serotype coverage can be improved if we understand the epidemic dynamics of IPD. It could have been possible that administration of vaccine at the nadir of an epidemic cycle, with measurement of attack rate at the apex of a subsequent cycle, could give a false impression of poor vaccine efficacy, when the increased incidence of IPD was part of a predictable natural phenomenon. Controlling for this would clarify the relationship between the vaccine and surrounding, confounding conditions. As no periodicity exists, this can be excluded as a factor in efficacy, and researchers can target other areas impacting vaccine efficacy, such as molecular factors (Obert et al., 2006).

\section{Summary}

This introduction provided a brief examination of the current state of knowledge addressing IPD. The characteristics of the bacterium that facilitate colonization and infection were addressed, with an emphasis on the endemicity of pneumococcal colonization. Additionally, the basic concepts of infectious disease dynamics, specifically seasonality and periodicity, were introduced. The use of mathematical modeling to describe and understand disease dynamics was briefly explained, and a potential approach to determining or refuting the existence of a periodic epidemic pattern in the 
disease dynamics of IPD was presented. Definitions for uncommon terms have been provided in this introductory chapter, as well as the hypotheses, assumptions, and boundaries for the study. Finally, a discussion of the positive social change that could result from an investigation into IPD epidemic dynamics was offered. Further explanation and clarification will be found in subsequent chapters.

Invasive pneumococcal disease presents a significant risk to vulnerable subpopulations, despite an extensive body of knowledge covering its causal factors, atrisk hosts, successful protocols for treatment, and vaccine-based prevention. Much remains to be uncovered about IPD, particularly whether or not IPD epidemics occur in a predictable, periodic manner. This research yields information on the absence of perturbations in the epidemiologic pattern over the study period, when compared to the normal incidence rate. These perturbations would have constituted evidence of large epidemics of invasive disease, and if present would have been examined and analyzed to establish the existence of epidemic periodicity, and the frequency of that periodicity or aperiodicity The expected outcome was establishment of whether IPD occurs in predictable epidemics over the long term, in all ages, genders, and geographic areas. The results of this study provide information valuable to other researchers, and could generate many other lines of inquiry. To further understand the extent of our current understanding of IPD, and the methods that may be applied to identification of possible epidemic patterns, a more in-depth and thorough review of the literature follows. Chapter 3 contains a discussion of the study methods. 


\section{CHAPTER 2: REVIEW OF THE LITERATURE}

\section{Introduction and Background}

In the United States, Invasive Pneumococcal Disease (IPD) is responsible for approximately forty thousand deaths each year from pneumococcal infection of all types (Bloom, Wheeler, \& Lynn, 1999; McDaniel \& Swialto, 2004), with an estimated 175,000 hospitalizations due to the disease annually (CDC, 2006). This disease is not evenly distributed throughout the population in the United States. The very young, the old, those with immunonological deficiencies, and indigenous groups bear the major burden of this disease (Butler, 2004). Variation within subpopulations exists in IPD incidence, with children (McNabb et al., 2007), African Americans (Robinson et al., 2001), Alaska Natives (Butler \& Schuchat, 1999), and Native Americans (Benin et al., 2003) among the high-risk groups for the disease.

A great deal of knowledge has been accumulated on IPD, encompassing the natural history of the responsible pathogen, the subpopulations at risk for IPD, and the treatment of IPD. The study of past efforts into understanding the pathology and epidemiology of $S$. pneumoniae establishes a basis for reflection on the many current inquiries focused on the pneumococcus. Understanding the organism and its epidemiology, developing and assessing clinical approaches to the treatment of pneumococcal disease, and prevention of the disease are lines of inquiry reflective of past research, present efforts, and future endeavors. The character of current inquiry into invasive diseases resulting from $S$. pneumoniae infection falls largely within the confines of those lines of inquiry. Much of the recent work concentrates on extending the work on clinical treatment, vaccine efficacy, vaccine delivery, microbial genetics and antibiotic 
resistance (Castillo, Rickman, Brodine, Ledbetter, \& Kelly, 2000; Guchev et al., 2005; Metlay et al., 2006). What is not understood is the epidemic pattern of IPD. While seasonality has been established, the pattern of epidemics of IPD has not.

The following chapter presents a critical review of the literature, to establish the current state of understanding of IPD. Key words used in the literature search included pneumococcus, Streptococcus pneumoniae, invasive pneumococcal disease, pneumococcal bacteremia, time-series analysis, epidemiologic modeling, and disease modeling. Common abbreviations were also identified, such as IPD. To enhance the value of returned searches, key words were grouped into concepts using Boolean operators (and/or/not), for instance, pneumococcal AND modeling.

The primary databases used were PUBMED, MEDLINE, the Cumulative Index of Nursing and Allied Health (CINAHL), ScienceDirect, and EBSCO Academic Search Premier. Returned searches were evaluated for literature that addressed the general research goals, and these documents were obtained, either by electronic download, interlibrary loan, or in some cases by purchase from the publisher. The literature was then categorized and cataloged using EndNote X1 for Mac (Thompson Reuters, Philadelphia, PA) bibliographic software.

The literature search proceeded in an iterative fashion. The list of references in each obtained document was used to identify additional literature, in particular primary sources, and to identify additional key words and concepts to further direct the search. To aid in the identification of new literature, an automated search of the PUBMED database was conducted weekly using the myNCBI feature and previously validated search strategies. 
In order to move towards an understanding of the epidemic pattern of IPD, it is first necessary to address our current understanding of IPD. This review of the literature provides a historical background and examines the pathogen itself. The review addresses the epidemiology of IPD, the subpopulations at risk, and the prevention of IPD. Much of the biology and epidemiology of $S$. pneumoniae is the basis by which the bacterium survives the host immune response (Grenfell et al., 2004) and maintains an endemic presence in the host. Variations in S. pneumoniae serotype, drug resistance, alterations in host/pathogen relationships, and variations in preventive measures are all part of infection dynamics, which over time is a major determinant of epidemic behavior (Grenfell et al.), and a clear understanding of these elements is needed as a basis for the study. A review of other diseases with epidemic periodicity follows, accompanied by a discussion of the mathematical modeling that has been employed to find epidemic periods in other diseases.

Of particular pertinence to the research is past work on the epidemiology of IPD, wherein the annual seasonality of $S$. pneumoniae invasive infection has been established (Dowell, Whitney, Wright, Rose, \& Schuchatt, 2003). However, patterns of large epidemics, that is, the existence or absence of a long-term recurring pattern to epidemic S. pneumoniae, have not been properly addressed. Currently, there is a dearth of research on large epidemic patterns in S. pneumoniae invasive infections; thus, public health and clinical practitioners lack the fundamental knowledge to make the appropriate choice of predictive models for long-term planning, and epidemics may go unacknowledged or their severity unrecognized when they deviate from the normal pattern or rate of prevalence. Furthermore, identification of epidemic patterns can aid in the assessment of 
vaccine effectiveness; that is, the extent to which the current vaccines address the most common infectious serotypes. If the epidemic pattern changes, or if the epidemic pattern has been eliminated by successful vaccines and vaccination programs, a pattern reemerges; a variation in an established pattern might alert practitioners to a change in pneumococcal epidemiology that requires attention and action. Epidemic pattern changes were noted subsequent to the implementation of measles vaccine (Noah, 1989), and a deviation from the normal periodicity alerted the public health community to changes in pertussis epidemiology (Galanis, King, Vaughese, \& Halperin, 2006). However, in both of these examples, the long-term periodic pattern of epidemics was known. The pattern, if any, of pneumococcal outbreaks has yet to be established. It is important in light of $S$. pneumoniae's endemicity and potential lethality that this does not continue. It is this gap in our knowledge that the study will fill.

Currently, interventions targeted to the prevention of pneumococcal disease emphasize vaccination (Dagan \& Lipsitch, 2004). However, reductions in funding for children's health and disparate patterns in realized access to health care (including vaccinations) hinder our ability to promote vaccination as an intervention. Establishment of a pattern to IPD epidemics could facilitate prediction of outbreaks. Vaccination promotion efforts could use this information to create incentives to vaccination (e.g., unvaccinated individuals might well seek vaccination in the face of a predicted epidemic) (Bauch, 2005; Bauch \& Earn, 2004). Additionally, this information might lead to enhanced disease surveillance through the ability to employ long-run probability calculation (Kachigan, 1982) for epidemic forecasting, and two, the ability to detect the atypical patterns of epidemics (Gertz et al., 2003; Hsieh et al., 2006). In order to justify 
this statement claiming positive social change implications for this research, and to clearly delineate the gap in the current knowledge regarding epidemic periodicity in IPD, a review of the literature follows, starting with an examination of S. pneumoniae as a significant human pathogen of historical importance.

Streptococcus pneumoniae as a Significant Human Pathogen of Historical Importance.

S. pneumoniae is a gram-positive pneumococcal bacterium identified almost simultaneously by Pasteur and Sternberg in 1880 (Austrian, 2000; Bridy-Pappas, Margolis, Center, \& Isaacman, 2005 \& Isaacman, 2005; Gray, 2000; Pasteur, 1881; Sternberg, 1881). Ubiquitous and highly adaptive, this bacterium has levied an enormous burden on its human hosts. Responsible for a variety of life-threatening diseases (Tomasz, 1999), S. pneumoniae is a frequent cause of Community-Acquired Pneumonia. Additionally, S. pneumoniae is the causal agent for a variety of invasive diseases that bear a high mortality rate, often attacking the most vulnerable members of a population, accounting for a large burden of disease, disability, and death.

The subject of concentrated study for over 128 years, much of S. pneumoniae's natural history and epidemiology is known, but much remains to be discovered. The history of our endeavors against S. pneumoniae is one of advances in understanding, from the genetic profile of the 92 distinct serotypes, to identification of at-risk populations, to development of efficacious antibiotics and vaccines.

The natural history of the pneumococcus and IPD has evolved through several distinct but overlapping periods; the first an initial period of microbial identification and establishment of a basic nomenclature and pathology, followed by a period emphasizing treatment, composed of pre- and post-antibiotic phases, a period focused on vaccination, 
both pediatric and adult, and finally, research into the pneumococcal genome. The first steps towards understanding the microbiology, pathology, pathophysiology, and epidemiology of S. pneumoniae were taken when Sternberg and Pasteur isolated the pneumococcus from human saliva (Sternberg from his own, and Pasteur from a pediatric victim of rabies), and later grew the organism in vitro (Austrian, 1960). Subsequently given a number of names, among them Microbe septicemique du salive, Mircoccus pasteuri, Bacillus saivarius septicus, Micrococcus pneumiae cruposae, Diplococcus pneumoniae, Micrococcus or Diplococcus lanceatolus, Micrococcus of sputum septicemia and Frankel's pneumococcus (also Pneumobacillus of Frankel), prior to settling on the now standard Streptococcus pneumoniae in 1974 (Jordan, 1918; Watson, Musher, Jacobson, \& Verhoef, 1993; Welch, 1892), the pneumococcus was the focus of many researchers, but it remained for Weichselbaum to identify the pneumococcus as the pathogen responsible for lobar pneumonia (Austrian, 1960). Soon after Weichselbaum's recognition of S. pneumoniae's role in lobar pneumonia, the pneumococcus was found to have invasive characteristics, demonstrating pathology outside of the pulmonary system. By the end of the 1880s, S. pneumoniae had been found in blood, cerebrospinal fluid (CSF), cardiac valves, joint fluid, and the middle ear (Austrian, 1981). The clinical and epidemiologic aspects of pneumococcal infection were soon documented; Osler (1899) described the signs, symptoms, and risk groups for lobar pneumonia only three years after Weichselbaum's work. A variety of researchers contributed to the extensive body of knowledge surrounding the pneumococcus, developing the ways and means to identify the pneumococcus in pathological samples and methods to assign a serotype to isolated bacterium. Neufeld, in particular, expanded our knowledge of the pneumococcus, 
describing the Quellung reaction (describing the swelling of the bacterium in the face of antiserum, and still used for typing of $S$. pneumoniae) and capsular types 1 and 2;

Haendel, Dochez and Gillespie added to Neufeld's work by defining a third capsular type (Austrian, 1999; Austrian, 2004; Watson et al., 1993). The next and overlapping phase of discovery addressed the clinical aspects of pneumococcal disease, both in its pneumonia and invasive manifestations.

Prior to widespread deployment of antibiotics, the use of horse and rabbit antisera was the primary therapeutic regimen for treatment of invasive pneumococcal disease (Austrian, 1999), requiring serotyping of the specific pneumococcus of clinical interest in order to choose the appropriate antisera (Howard, 1921). An enzyme derived from a related bacterium was found to selectively kill serotype $3 S$. pneumoniae, raising hopes for another pharmacotherapy; however, the enzyme levels required in vivo were extremely high, and the enzyme was only effective against serotype 3 , and not the more virulent serotype 1 (Avery \& Dubos, 1931). Thus, antisera remained the sole pharmacologic intervention, with the result that IPD commonly exhibited multi-organ system involvement and a high mortality, despite up to $50 \%$ mortality reductions through the use of equine antisera therapy (Howard, 1921). An example of multi-organ system invasive pneumococcal disease reflecting the pre-antibiotic period is the triad of pneumococcal pneumonia, meningitis, and endocarditis originally described in 1881, and later named Austrian's Triad (Austrian, 1957; Beadsworth, Wooton, Chenzbraun, \& Beaching, 2007; Taylor \& Sanders, 1999). Treatment of pneumococcal disease by antisera was short-lived as a clinical approach, as antibiotic development occurred very soon after the implementation of antisera therapy. The development of effective 
antibiotics was clearly a boon to the patient and the clinician confronted with pneumococcal disease, as before the availability of effective antibiotics, invasive pneumococcal disease killed over 70\% of those affected (Butler \& Cetron, 1999). Identification of antibiotics suitable for employment against the pneumococcus occurred soon after the discovery of the organism and its role in pneumonia and invasive disease. The first antibiotic deployed against S. pneumoniae, ethylhydrocuprein (optochin) (Watson et al., 1993), is notable for engendering the first cases of microbial resistance to antibiotics (Austrian, 2004), a situation that would be repeated. Because of this acquired resistance, and toxicity in humans, optochin was rendered of utility only as a laboratory test for identification of the pneumococcus (Bowers \& Jeffries, 1955). Other antibiotics followed, with penicillin initially proving effective against all serotypes of the pneumococcus, with the unfortunate result of delaying the development of efficacious vaccines and reducing interest in the pneumococcus as a clinical entity (Austrian, 2004; Austrian, 1960). Antibiotic therapy with sulfanilamides, penicillin, chloramphenacol, and chlortetracycline lowered case fatality rates from pneumococcal infections to $5 \%$ to $8 \%$, and clinical entities such as Austrian's Triad became rarer. Sources of pneumococcal typing sera at the commercial level were gone within a decade of the introduction of antibiotic therapy, as it was no longer profitable to make these products in the postpenicillin era (Austrian, 1999). However, the ability of S. pneumoniae to quickly develop antibiotic resistance led to renewed interest in vaccine development.

Pneumococcal vaccine development can be traced back to 1882 , one year after the isolation of S. pneumoniae, with the first large clinical trial of a pneumococcal vaccine occurring in 1911 (Austrian, 1978; Williams \& Masterton, 2008), with development of 
newer vaccines continuing throughout the 1940s, though vaccine development was slowed by the success of antibiotic therapies that promised to eliminate the threat of pneumococcal disease. However, when clinicians realized that IPD still carried a 25\% mortality rate, development of an effective polyvalent pneumococcal polysaccharide vaccine was undertaken (Bridy-Pappas, Margolis, Center, \& Isaacman, 2005; Butler, Shapiro, \& Carlone, 1999; Whitney, Schaffner, \& Butler, 2001).

From this brief overview, it is clear that the study of S. pneumoniae has contributed to our understanding of genetics, antimicrobials, and development of microbiological laboratory procedures. If those were the only outcomes associated with our study of the pneumococcus, it would still remain worthy of our attention, as $S$. pneumoniae is an important agent and cause of life-threatening disease, and more knowledge of its epidemiology is required. Our current understanding of the nature of its most serious manifestation, IPD, is examined next.

\section{General Epidemiology}

\section{Characteristics of the Causal Agent for IPD, Streptococcus pneumoniae}

The causal agent for IPD is the bacterium $S$. pneumoniae, a gram-positive, alphahemolytic, catalase-negative, anaerobic bacterium (Bridy-Pappas et al., 2005). $S$. pneumoniae colonization is endemic (Rieux, 2002). This endemicity is important to this study, as endemic levels of colonization are required for IPD, and endemic IPD will accompany any IPD epidemics and epidemic patterns that might be detected. To understand the mechanism that enables $S$. pneumoniae to achieve all of the feats ascribed to it, despite vaccines and antibiotics targeted against it, a thorough review of the microbiology of $S$. pneumoniae is in order. 
The bacterium colonizes the nasopharynx, and is normal flora and nonpathological in most individuals (Rieux, 2002). In most cases, colonization with $S$. pneumoniae is asymptomatic (Alekshun \& Levy, 2006; Obert et al., 2006). The pneumococcus is a primarily human-restricted commensal that will be acquired at one point or another by the majority of the population (Bridy-Pappas et al., 2005; Sleeman et al., 2006). There is evidence supporting S. pneumoniae as a primary pathogen in horses (Serotype 3); however, the equine reservoir does not present a host or vector for human colonization or infection (Whatmore et al., 1999). Therefore, for the remainder of this review, only human colonization and disease are considered.

Nearly all children experience bacterial colonization and carriage during the first two years of life (Brueggemann, Peto, Crook, Butler, \& Kristinsson, 2004; Hammitt et al., 2006). Colonization with S. pneumoniae may occur on the day of birth, and children may be colonized with as many as four serotypes at one time (Gundel \& Schwarz, 1932). Colonization peaks sometime during the second or third year of life and this near universal pediatric colonization is a key factor in the endemicity and transmission of the bacterium (Myers \& Gervaix, 2007). Colonization is common to such a degree that Berg et al. (2006) consider the pneumococcus to be normal flora of the nasopharynx and upper respiratory tract. Carriage rates in healthy adults range up to $30 \%$, in healthy children, from to 50 to $60 \%$. This colonization can persist up to four months. However, carriage periods average only two to four weeks for most adults (Bridy-Pappas et al., 2005; Müller-Graf et al., 1999; Myers \& Gervaix, 2007). As long as the colonizing serotypes are not virulent strains, continued colonization might not be a negative finding, as it appears that colonization with S. pneumoniae may inhibit nasal colonization by 
Staphylococcus aureus and Haemophilus influenzae, primarily through inhibition via production of hydrogen peroxide (Pericone, Overweg, Hermans, \& Weiser, 2000; RegevYochay, Trzcinksi, Thompson, Malley, \& Lipsitch, 2006). Exposure to $\mathrm{H}_{2} \mathrm{O}_{2}$ is lethal to the Staphylococcus; however, S. pneumoniae is not inhibited by endogenously produced $\mathrm{H}_{2} \mathrm{O}_{2}$, regardless of an increased bacterial mutation rate (Pericone et al., 2003).

It is clear that colonization with $S$. pneumoniae is more often than not a normal, benign condition. However, colonization is a necessary step towards the acquisition of IPD (Nelson et al., 2007), and because of the near universality of colonization, IPD should not be susceptible to extinction. Universal colonization does not provide a mechanism by which IPD epidemics might display periodicity, but does provide the mechanism by which the disease can develop and remain endemic.

When S. pneumoniae leaves its normal ecological niche, clinical illness (often life-threatening) might be the result (Pericone et al., 2003). Dwelling in the host nasopharynx is advantageous to the pneumoccocus, as it permits transmission to other susceptible hosts. Leaving the nasopharynx and acting in a pathogenic manner is not required for bacterial life or reproduction, and fatal pathogenesis might actually be disadvantageous to the pneumococcus, as the death of a carrier precludes further spread of the organism (Briles, Novak, Hotomi, Ginkel, \& King, 2005). However, the ability of virulent strains of $S$. pneumoniae to evade host defenses should confer some advantage over less virulent strains within the same host. The selection pressures within the individual host might encourage the growth of bacterium that successfully evades host immune responses. This creates a situation in which the more virulent strain exchanges transmissibility from the host for the ability to reproduce (Lipsitch \& Moxon, 1997). 
Whether as a result of host selection pressure or not, the pneumococcus does leave its normal ecological niche and results in disease in some vulnerable hosts. How the pneumococcus leaves the nasopharynx, evades the host immune responses and invades otherwise sterile sites is directly related to bacterial morphology, and provides the biologic mechanism needed for acquisition of IPD in the susceptible host.

S. pneumoniae morphology can be described by the conformation of a polysaccharide capsule (Austrian, 1954). Each S. pneumoniae strain can be categorized by capsular serotype, based on the laboratory identification of one or more of at least 92 immunologically and chemically distinct polysaccharide capsules (Obert et al., 2006; Rieux, 2002). These serotypes may be immunologically cross-reactive, but many are not, creating challenges for both the host immune systems and vaccine development (Hausdorff, Bryant, Kloek, Paradiso, \& Siber, 2000). Within serotypes, a number of genetically different clones may be identified by means of molecular genetic techniques (Jefferies, Smith, Clark, Dowson, \& Mitchell, 2004). This within serotype diversity may be due to the ability of $S$. pneumoniae to horizontally transfer genetic material (Brueggemann et al., 2003; Carrolo, Pinto, Melo-Cristino, \& Ramirez, 2009; Lopez, 2006). This ability to harbor distinctive clones within serotypes accounts for the presence of both non-resistant and drug-resistant strains within defined serotypes. The wide variability in composition of the capsule in each serotype has also given rise to speculation that the human immune system views each serotype of $S$. pneumoniae as a different pathogen (Lipsitch et al., 2005). This presents a plausible explanation for the lack of cross-coverage between vaccine and non-vaccine types, and for the persistence of the bacterium in its hosts. 
Capsulation is required for invasiveness, with different strains varying in invasiveness based upon capsular serotype (Sleeman et al., 2006). Invasiveness can vary as much as 60 times between the least and most invasive serotypes (Crook, 2006), and the nature of the capsular type can determine whether the pneumococcus behaves as a primary or opportunistic pathogen (Sjoestroem et al., 2006). It has long been recognized that non-capsulated avirulent strains can mutate into capsulated, virulent strains (Avery, MacLeod, \& McCarty, 1944). Of note, several other bacterium that are linked to bacterial meningitis are encapsulated, Haemophilus influenzae type $\mathrm{b}$ and Neisseria meningitis, for example. This encapsulation is required if the bacterium is to breach the blood-brain barrier (Nelson et al., 2007), thus accounting for the role of S. pneumoniae as a cause of the meningococcal form of IPD.

A competent pneumococcal polysaccharide capsule is part defense mechanism (Avery \& Dubos, 1931), a determinant of virulence and morbidity (Rytel, Dee, Ferstenfeld, \& Hensley, 1974), a target for vaccines (Lipsitch et al., 2005) and a means of specific type identification. The major virulence factor for $S$. pneumoniae is the capsular polysaccharide, which defends the bacterium from opsonization and thus phagocytosis (Rieux, 2002; Varon, 2001). The capsule is composed of peptoglycans, and is both hydrophyllic and polarized, carrying a negative electrical charge (Rieux). It modulates ion transfer into the cellular membrane, and aids in the formation of biofilms (Allegrucci \& Sauer, 2007), as well as adhesion to the epithelium of the nasopharynx (Varon, 2001). Biofilm formation in S. pneumoniae may aid in persistence of colonization, increasing the opportunity of the organism to spread to other vulnerable hosts (Moscoso, Garcia, \& Lopez, 2006). 
Capsular incompetence seriously reduces bacterial virulence, and nonencapsulated strains are avirulent (Kharat \& Tomasz, 2006). The capsule also presents antigens that are immunogenic. Successful immunization provides sufficiently high titers of antibodies to these capsular antigens, turning the bacterial protective strategy to an immunologic advantage.

In addition to capsular capability, isolates of S. pneumoniae exhibit three phenotypes, opaque, semi-transparent and transparent, when viewed on a transparent solid medium (Cundell, Weiser, Shen, Young, \& Tuomanen, 1995; King, Hippe, \& Weiser, 2006; Weiser, Austrian, Sreenivasan, \& Masure, 1994), or combinations thereof. This is not solely a descriptive phenomenon, as it appears that the ability of the pneumococcus to colonize a susceptible host is largely related to phenotype. Spontaneous and reversible variations in the phenotypes appear to be related to different stages of colonization and infection, and to potential for virulence (Serrano, Melo-Cristino, \& Ramirez, 2006; Woude \& Baumler, 2004). These phase variations are notable for differences in autolysis, production of techoic acid (required for cellular adhesion, and reduced by as much as half in opaque variants) and to site-specific adhesion (Allegrucci \& Sauer, 2007; Woude \& Baumler).

The transparent phenotype appears to be better suited to host nasopharyngeal mucosal cell surface adhesion, with an increased ability to desialylate host proteins such as C-reactive protein (most commonly in the acute phase of the infection (MacLeod \& Avery, 1941)) and immunoglobulins. The transparent variant exhibits twice the adherence capability of opaque phenotypes in vitro (Cundell et al., 1995).This increased 
adherence capability is as a result of capsular and cell wall structure (Magee \& Yother, 2001; Rajam et al., 2008; Tong, Weiser, James, \& DeMaria, 2001).

The opaque phenotype, with its resistance to opsonization and phagocytosis, is more suited to invasiveness outside of the respiratory tract (King et al., 2006; Sjoestroem et al., 2006). The robust capsule of the opaque variant is essential to invasiveness (Magee \& Yother, 2001), and the diminished capability of the capsule to adhere to mucosa permits bacterial escape from mucus and precludes bacterial clearance by mucocilliary flow (Nelson et al., 2007). Extrapulmonary invasiveness then proceeds via bacterial/host chemical interactions with the vascular endothelium of the alveolar capillaries, whereby disease may transition from pneumococcal pneumonia to the pneumococcal sepsis variant of IPD. Past studies have shown this process to occur in more than $20 \%$ of pneumococcal pneumonia cases (Andonegui, Goring, Liu, McCafferty, \& Winston, 2009; Ortqvist et al., 1990).

The opaque phase variants might also escape the nasopharynx via the cervical lymphatics (Weiser et al., 1994). When invading via the pulmonary vasculature, the transition to invasive pathogen is facilitated by cytokine stimulation of the vascular endothelium; opaque phenotype adherence in these cell types results partially from interactions between pneumococcal cell wall techoic acid and a related phosphorylcholine determinant (PCho) in de novo Platelet-Activating Factor (PAF) in the host epithelial cells. Binding to PAF aids the pneumococcus in migration through the respiratory and vascular epithelium. Of note, PAF receptors have also been identified in the brain (Cundell et al., 1995; Garau, Lemaire, Vernet, Dideberg, \& Guilmi, 2005). 
In addition to the action of techoic acid at PAF receptor sites, $S$. pneumoniae of all phenotypes produces surface exoglycosidases that promote adherence. A pneumococcal neuraminidase, NanA, working in sequence with a beta-galctosidase and a beta-N-acetylglucosaminidase, removes terminal sialic acids from the host cell, exposing additional adherence receptors and providing a carbon source for bacterial nutrition. As already noted, all morphological phases of $S$. pneumoniae produce NanA, but this ability to deglycosylate host proteins is of particular importance to adhesion in the trachea and other structures outside of the normal nasopharyngeal colonization sites (Hsiao, Parker, Ratner, Prince, \& Tong, 2009; King et al., 2006).

S. pneumoniae exhibits spontaneous phase variations between the three phenotypes (Cundell et al., 1995), thus permitting invasive and non-invasive clones within the same serotype (Obert et al., 2006). This has been observed in other human respiratory pathogens, such as Haemophilus and Neisseria spp. The ability to spontaneously shift phenotypic variables permits the pathogen to adapt to varying challenges presented by the host immune response in the different ecologies of the nasopharynx, pulmonary structures, blood, or cerebral spinal fluid (Cundell et al., 1995). Transparent variants are successful at adhering to nasopharyngeal cells, while opaque variants are capable of evading mucocilliary clearance, travel from the mucosa to the epithelium, and thereby ensure that a part of the bacterial growth can evade mucosalbased immunologic responses (Bootsma, Egmont-Peterson, \& Hermans, 2007; Nelson et al., 2007).

Three hundred and ninety seven genes that appear to be conserved across strains govern phase variation in S. pneumoniae, making the ability of $S$. pneumoniae strains to 
engage in invasiveness dependent on genome content. In the laboratory setting, a variety of techniques have been developed to classify pneumococcal isolates on a genetic basis.

In vivo, however, it is environmental conditions that result in varied expression of capsular and other pneumococcal genes (Obert, Gao, Sublett, Tuomanen, \& Orihuela, 2007; Ohlsen, Hacker, \& Ziebuhr, 2004), and pneumococcal gene expression varies depending on the site of colonization (Orihuela et al., 2004). In blood, S. pneumoniae reacts as if under stress and in a nutrient poor environment, despite the nutrient-rich medium presented by blood. However, it is clear that the pneumoccocus takes advantage of the high carbon availability in blood, as the organism reduces production of substrates needed for sucrose and maltose metabolism (Orihuela et al.). In CSF, different gene expressions occur, also consistent with an organism under stress in a nutrient-poor environment (Orihuela et al.).

Given the importance of adherence to successful bacterial colonization, it should come as no surprise that $S$. pneumoniae exercises additional measures beyond modification of host cell surface biochemistry to ensure that adhesion takes place. In some clinical isolates, pili extend beyond the polysaccharide capsule, enhancing adhesion to the pulmonary epithelium. This increased adhesion increases virulence, but not without a cost to the microbe. The presence of pili in a systemic infection stimulates a higher TNF response, with an accompanying higher host inflammatory response, and might thus negatively impact bacterial survival (Barocchi et al., 2006; Regev-Yochay, Lipsitch, Basset, Rubenstein, Dagan, Raz et al., 2009). 
Through this wide variety of measures, the pneumococcus may invade normally sterile sites. The results of these morphology-mediated invasions are then dependent on pneumococcal virulence factors and the host's ability to respond to the invasive disease. Virulence is based on a combination of intrinsic factors of the bacterium, and extrinsic factors related to the host's immune function (Rieux, 2002). Virulence factors are genetically determined, and include capsular composition, pneumolysin (SP1923), hemolytic cytotoxin, autolysin (SP1937), major murein hydrolase, pyruvate oxidase (SP0930), Pneumococcal Surface Antigen A (PsaA), Pneumococcal Surface Protein A (PspA), and a heat shock serine protease, HtrA, among others (Echenique, Kadioglu, Romao, Andrew, \& Trombe, 2004; Jedrzejas, 2001; Linder, Hollingshead, Januczyk, Christensson, \& Akesson, 2007; Obert et al., 2006; Rieux, 2002). Genes expressing these factors have been shown as essential for colonization and invasive disease, and are part of the core genome of S. pneumoniae (Obert et al., 2006; Ohlsen et al., 2004)). The presence of the polysaccharide capsule is regarded as the primary virulence factor, but several other pneumococcal proteins count as virulence factors (Jedrzejas, 2001).

Genomic sequencing has been completed for three pneumococcal strains, two virulent (serotype 4, specifically the TIGR4 strain; and serotype 19F, specifically the G54 strain) and the other avirulent (serotype 39, the laboratory strain R6), and partial sequencing has been completed for several other serotypes (Dopazo et al., 2001; Lanie et al., 2007; Tettelin et al., 2001). The pneumococcus genotype is highly plastic, capable of sharing genes horizontally between both its own species and other closely related bacterial species. It is this horizontal transfer that has been credited with the development of penicillin resistance by the pneumococcus (Dowson, Coffey, \& Spratt, 1994; 
Hakenbeck et al., 2001; Hsieh et al., 2006). This genetic plasticity and high level of adaptability exists in all serotypes of $S$. pneumoniae and again, may account for both emergence of a specific serotype in an epidemic, for antibiotic resistance and for endemicity (Lopez, 2006). However, serotype distribution differs by geographic area and age group (Babl, Pelton, Theodore, \& Klein, 2000; Sjoestroem et al., 2006).

Certain serotypes are associated with high attack rates and are rarely isolated from healthy carriers; conversely, other serotypes are often isolated from healthy carriers but have very low attack rates. A small subset of serotypes causes most invasive pneumococcal disease (Bridy-Pappas et al., 2005; Hausdorff, Feikin, \& Klugman, 2005; Lencastre \& Tomasz, 2002; Obert et al., 2006; Sleeman et al., 2006), with attack rates varying by capsular serotype (Sleeman et al.); it is believed that an estimated $90 \%$ of IPD is caused by only 16 distinct serotypes (Hakenbeck et al., 2001). Almost $90 \%$ of pneumococcal invasive disease in children can be attributed to eleven serogroups, serogroups 1,3,4,5,6,7,9,14, 18,19, and 23 (Babl, Pelton, Theodore, \& Klein, 2001; Hausdorff, 2007; Kyaw, Clarke, Edwards, Jones, \& Campell, 2000). Patients infected with HIV are often infected with these same serogroups (Babl, Pelton, Theodore, \& Klein). The most prevalent serogroups in adults are 1,9,14,7,4,23,3,8,6,19 and 22 (Kyaw et al.). Additionally, higher rates of mortality are associated with specific serotypes; invasive disease caused by serotypes $3,6 \mathrm{~B}$ and $19 \mathrm{~F}$ have higher mortality than that caused by serotypes 1 and 7F (25\% and 10\%, respectively) (Sjoestroem et al., 2006). Furthermore, some virulent serotypes (1 and 7F) infect otherwise healthy individuals, functioning as primary pathogens, while other serotypes cause disease in subjects with predisposing conditions, acting as opportunistic pathogens (Sjoestroem et al). 
Mode of Transmission

Pneumococci are transmitted by airborne droplets; viable bacteria have been isolated in the air of crowded rooms after sneezing (Bridy-Pappas et al., 2005; Berk, Gage, Holtsclaw-Berk, \& Smith, 1985). Outbreaks of pneumococcal disease often occur in settings of crowding (Greenwood, 1999), pointing towards a density dependence in $S$. pneumoniae endemicity and epidemic potential. Outbreaks in jails, military training settings, nursing homes, and child-care centers are well documented (Butler, Dowell, \& Breiman, 1998; Hoge et al., 1994). Because of the droplet transmission and the level of endemicity of S. pneumoniae, exposure in these close communities is universal and periodic colonization and re-colonization guaranteed (Gordon \& French, 2005). Stress predisposes subjects to infection, with sleep and calorie deprivation contributing to the incidence of IPD (Butler \& Schuchat, 1999).

Incidence and Prevalence of IPD in the United States General burden of IPD on the Human Population

Despite our extensive knowledge of the pneumococcus, and the availability of effective antibiotics to fight invasive pneumococcal infection, invasive infections resulting from S. pneumoniae present a significant burden to the human population. $S$. pneumoniae is the leading cause of bacteremia, sepsis and bacterial meningitis (with an incidence rate of 1.1/100,000) in the United States (Bridy-Pappas et al., 2005); the relationship between S. pneumoniae and meningitis is particularly noteworthy, as, Haemophilus influenzae type $\mathrm{b}(\mathrm{Hib})$ infections have declined in the U.S., due to the availability of an efficacious vaccine against that pathogen (Bridy-Pappas et al.; Butler, Shapiro, \& Carlone, 1999; Cabellos et al., 2009; Chen et al., 1998; Lanie et al., 2007; 
Obert et al., 2006); post-Hib vaccine, S. pneumoniae emerged as the dominant cause of bacterial invasive diseases in children, including meningitis (Bernatoniene \& Finn, 2005; Hamdad et al., 2007; Overturf, 2000).

In terms of morbidity and mortality, the negative consequences of invasive pneumococcal disease are significant, as disease caused by $S$. pneumoniae has been reported as responsible for an estimated $36 \%$ of community-acquired pneumonia, $50 \%$ of nosocomial pneumonias. Also, it is responsible for 50,000 cases of bacteremia, and 3,000-6,000 cases of meningitis each year in the United States (CDC, 2006). In the United States, approximately 40,000 deaths have been reported each year from pneumococcal infection of all types (pneumonia and invasive) (Bloom et al., 1999; McDaniel \& Swialto, 2004), with an estimated 175,000 hospitalizations due to the disease each year in the United States (CDC). Greater than half of the invasive pneumococcal disease in the United States occurs in the population with chronic disease (Kyaw et al., 2005).

A 2001 study by Robinson et al. established that invasive pneumococcal disease could be responsible for more than 62,000 hospitalizations and more than 6,000 deaths in 1998 alone (Robinson et al.). These deaths most often occur in the older population. The mortality rate for invasive pneumococcal disease is highest among those aged over 80 years, at 17.4/100,000 (Robinson et al.). However, other subpopulations share in the general burden of invasive pneumococcal disease. Case fatality rates are highest for the subpopulations with chronic diseases, immunosuppression, congestive heart failure, malignancy, renal failure, and HIV/AIDS (Alanee et al., 2007; Robinson et al.). The 
mortality of pneumococcal meningitis approaches $30 \%$ and half of the survivors suffer permanent neurological deficits (Orihuela \& Tuomanen, 2006).

Incidence of Invasive Pneumococcal Infections in the United States

The incidence of IPD varies across the US, with different communities displaying widely different rates (Huang, Finkelstein, \& Lipstich, 2005). This variation in rate may be due to differences in contact patterns (Bansal, Grenfell, \& Meyers, 2007). These different rates of incidence (and the plasticity of the organism) may serve to prevent complete eradication of $S$. pneumoniae colonization, as between patch transmission prevents local extinctions from becoming global extinctions (Hagenaars, Donnelly, \& Ferguson, 2004). Invasive pneumococcal disease displays geographic variance in incidence rates and serotype frequency (Hausdorff, Silber, \& Paradiso, 2001; Sniadack et al., 1995), still, across geographic regions, the rates of invasive pneumococcal disease are highest in the first two years of life (Butler, Shapiro, \& Carlone, 1999), with documented incidence rates in the post-conjugate vaccine period at 8-75 cases per 100,000 (Brueggemann, Peto, Crook, Butler, \& Kristinsson, 2004).

Incidence rates vary by sub-population. In adults in long-term care facilities, the incidence rate of invasive pneumococcal disease is 194.2 cases per 100,000 , compared to a rate of 44.6/100,000 for the same age group living in the community (Bridy-Pappas et al., 2005). As another example of variation within subpopulations, among the Navaho, incidence rates of 78-235 cases per 100,000 annually have been observed; elevated incidence rates have also been observed in the White Mountain Apache tribe (Benin et al., 2003). 
IPD rates are markedly higher among Native Americans/Alaskan natives, who at some points in time have had some of the world's highest rates of invasive pneumococcal disease (Rudolph, Parkinson, Reasonover, Parks, \& Butler, 2000), with an age-adjusted incidence rate 2.5 times higher than non-Alaska Natives in the same geographic area (Hennessy et al., 2002), who have similar incidence rates to overall US rates (Hennessy et al., 2005). For Alaskan Natives 65 years old or older, the incidence rate for invasive pneumococcal infections has been documented at 186/100,000 (Butler \& Schuchat, 1999). No data supporting specific genetic factors predisposing indigenous populations to invasive pneumococcal disease has been published (Butler et al., 2001), and associations between socioeconomic status (SES) and IPD, which could account for the higher incidence, have been refuted in another study (Chen et al., 1998). The higher overall incidence of IPD in this population may be more due to health factors and personal habits than with SES or environmental conditions (Singleton et al., 2007). The prevalence of drug-resistant pneumococcal serotypes is also high in these populations, with over $10 \%$ of pneumococcal isolates having reduced susceptibility to ß-lactam antibiotics during the 1990’s (Hennessy et al., 2002).

The incidence of invasive pneumococcal disease is also higher in African Americans than in the general population, regardless of income strata (Chen et al., 1998). African Americans had an overall incidence of invasive pneumococcal disease of 49.7/100,000, compared with an overall incidence rate for whites of 19.7/100,000 (Robinson et al., 2001). By 2000, the overall incidence rate was 8.8 per 100,000 in otherwise healthy adults. In the subpopulation of adults aged between 65 and 79, the incidence rate was 23.3 per 100,000, and for adults aged 80 and greater, 71.2/100,000 
(Kyaw et al., 2005). Hospitalization rates fell as well, with a discharge rate of 9.12/100,000 in 1998 and a 2003 rate of 5.6/100,000 (Shah \& Ratner).

HIV-positive persons are another subpopulation with disparate incidence rates of IPD. Due to the variation in pneumococcal capsule, the already impaired immune system of the HIV-positive person may not recognize even previously acquired serotypes, making recurrent IPD a factor in this population and in other immunocompromised groups (McEllistrem et al., 2002; Turett, Blum, \& Telzak, 2001). The peak of incidence for IPD in HIV-positive persons occurs between 35 and 49 years of age, with an incidence rate of 571.3 to 820.7 cases per 100,000 in these age groups (Kyaw et al., 2005; Steenhoff, Wood, Rutstein, Wahl, McGowan, \& Shah, 2008). Antibiotic resistant strains have been observed in increasing proportions in this population (Buie et al., 2004).

The annual attack rate for HIV-positive persons is 1\% (Butler \& Schuchat, 1999). In San Francisco during the 1990's, over 40\% of invasive pneumococcal disease in the population aged from 18-64 years was in HIV-positive persons (Butler et al., 1998). However, since the institution of Highly Active Antiretroviral Therapy (HAART), the incidence of invasive pneumococcal disease in the HIV-positive population has fallen, with a $57 \%$ decline in the first five years after the introduction of HAART. However, the incidence remains much higher than that of the general population, with estimates of up to 40 times more incidence, and higher levels of disease recurrence (Heffernan et al., 2005; Steenhoff, Wood, Rutstein, Wahl, McGowan, \& Shah, 2008).

Incidence rates for IPD in the United States have been declining since the introduction of the pediatric vaccine (Hammitt et al., 2006; McBean, Park, Caldwell, \& Yu, 2005; Moore et al., 2004; Poehling et al., 2006; Shah \& Ratner, 2005; Tsai, Griffin, 
Nuorti, \& Grijalva, 2008; Wilkinson, Bulloch, \& Smith, 2009). This reduction in incidence may be credited to the indirect effect of herd immunity (Haber et al., 2005; Millar et al., 2008; Tasslimi et al., 2008; Williams \& Masterton, 2008). As an example of a reduction in IPD incidence that might be credited to herd immunity, Rosen \& Ryan (2007) examined adult workers in a children's daycare facility, and found that these workers were less likely than other adults to be colonized with S. pneumoniae, and make a case that this is due to wide-spread pediatric vaccination. A study conducted by Grijalva, Nuorti, Arbogast, Martin, Edwards, and Griffin (2007) lends support to this conclusion.

In 1998, the overall incidence of IPD in the United States was 23.2 per 100,000 for all ages combined (Hammitt et al., 2006; Moore et al., 2004; Robinson et al., 2001; Shah \& Ratner, 2005). Incidence was highest among children younger than two, at 166.9 cases per 100,000. Incidence was lowest for the population aged from five to 17 , with a rate of 3.9 per 100,000 . The incidence increased again among the 65 and older age group, with an incidence rate of 61.5/100,000 (McBean et al., 2005).

As in the general population, the incidence of IPD in the Alaskan Native population has decreased since the introduction of the conjugate vaccine; decreases in IPD occurred as decreases in the serotypes included in the vaccine. In the Alaska Native population, pediatric pneumococcal meningitis declined from $6.0 / 100,000$ to $1.3 / 100,000$ in the post-vaccine period. This decrease occurred in the Alaska Native population even with continued carriage of pneumococcus, though carriage of serotypes addressed by the conjugate vaccine decreased (Hennessy et al., 2005; Miernyk et al., 2000; Moore et al., 2004). Evidence shows that the 23 -valent vaccine given to those over two years of age is 
also efficacious in the Alaskan Native population, and might further reduce the incidence of pneumococcal disease (Singleton et al., 2007).

The incidence rate for drug-resistant $S$. pneumoniae invasive infections in the subpopulation aged less than one year was 12.4/100,000; for adults greater than 65 years of age, 4.1/100,000 (McNabb et al., 2007). This declining incidence in drug-resistant infections may be attributable to better discretion in the use of antibiotics, the effects of vaccination campaigns in the pediatric and adult populations, age-specific immunological barriers, or a combination of these factors (Borer et al., 2001; McBean et al. 2005). Supporting this contention is the observation that drug-resistance in $S$. pneumoniae varies by geographic area, reflecting different antibiotic selection pressures (McBean et al.). Geographic variation should be considered when examining epidemic patterns of IPD, in addition to consideration when assessing drug-resistance patterns.

The effect of these reductions in IPD incidence is of importance to the concept of periodicity. It could be hypothesized that a reduction in overall incidence could preclude detection of IPD periodicity. In other words, as the incidence of epidemics is reduced to a level approaching the endemic level, periodic peaks may be harder to detect. However, lower amplitude of any periodic events will not prevent detection of these events by frequency domain analysis (Wei, 1994b), and the periodicity and frequency (if any) will remain detectable even in the face of under-reporting of events that might also account for the appearance of reduced incidence (Keeling \& Rohani, 2008b). S. pneumoniae Colonization and Infection, and Risk Factors for IPD

Colonization with S. pneumoniae is common (Rieux, 2002). Infection, however, is distributed in the population according to risk factors. As S. pneumoniae relies on 
airborne transmission, contact patterns between colonized and susceptible persons determine transmission patterns, and can provide selection pressures that force bacterial mutation (Petermann \& Rios, 2004; Wallinga, Edmunds, \& Kretzschmar, 1999). Prior to the availability of effective antibiotics, epidemics of pneumococcal disease were common in closed populations, such as prisons, military barracks, and dormitories (Hoge et al., 1994). Outbreaks within closed communities are less common today, but still occur, often in nursing homes and other residential health care settings (Butler \& Cetron, 1999). Long-term care facilities also provide a reservoir for $S$. pneumoniae (Butler \& Cetron), and day care centers have been identified as environments favorable for selection and amplification of S. pneumoniae, including drugresistant strains (Dagan et al., 2002; Lencastre \& Tomasz, 2002). Invasive pneumococcal infections have a bimodal age distribution, most often affecting children younger than five years and adults older than 65 years, reflecting both the unique immunology of those populations, and the nature of the environment where those subpopulations are often found. However, IPD can occur in any age group and is associated with a high mortality rate (Christenson, Lundbergh, Hedlund, \& Ortqvist, 2001; McDaniel \& Swialto, 2004).

The risk of IPD is highest among children, older adults, smokers, and the population with certain chronic diseases (Robinson et al., 2001; Butler \& Schuchat, 1999; Hjuler et al., 2008). The pediatric subpopulation has the highest incidence rates of IPD, with the highest levels among children aged less than two years (Robinson et al., 2001). However, the elderly and those with chronic diseases are also at risk; in the United States, these adult subpopulations contain the individuals who are also the most likely to die of 
IPD, even in the absence of risk factors other than age (Klemets, Lyytikainen, Ruutu, Ollgren, \& Nuorti, 2008; Whitney, Schaffner, \& Butler, 2001).

In addition to the risk of pneumococcal infection inherent to age, other risk factors increase vulnerability to pneumococcal infection. These risk factors are medical conditions that significantly increase a patient's vulnerability to pneumococcal pneumonia or IPD. The medical conditions are cardiovascular disease (Robinson, Baughman, Rothrock, Barrett, Pass, Lexau et al., 2001), cardiac transplant (Amber, Gilbert, Schiffman \& Jacobsen, 1990), cerebrovascular disease (Bridy-Pappas, Margolis, Center \& Issacman, 2005), lower and chronic respiratory diseases (Betts, 2003; Juhn, Kita, Yawn, Boyce, Yoo, McGree et al. 2008), renal disease, diabetes mellitus (Kyaw, Rose, Fry, Singleton, Moore, Zell et al., 2005), chronic alcoholism and its complications (Haddad, Poruczni, Joyce, De, Pavia, Rolfs et al., 2008), sickle cell disease (Klemets, Lyytikainen, Ruutu, Ollgren \& Nuorti, 2008), asplenia (either functional or anatomic) (Gray, 2000; Schutze, Mason, Barson, Kim, Wald, Givner et al., 2002; Smith, Erlandson, Shulman \& Stern, 1957; Torres \& Bisno, 1973), and malignant neoplasms (Berk, Gage, Holtsclaw-Berk \& Smith, 1985). Patients with these risk factors alone are considered eligible for pneumococcal vaccination if aged between two and 65 years (Christenson, Lundbergh, Hedlund, \& Örtqvist, 2001; Dworkin, Ward, Hanson, Jones, \& Kaplan, 2001; Gilbert et al., 2002; Fedson, Harward, Reid, \& Kaiser, 1990; Haddad et al., 2008; Maki, 2004; McDaniel \& Swialto, 2004; Whitney, Schaffner, and Butler, 2001).

Smoking and exposure to environmental tobacco smoke, are known risk factors for IPD (Breiman et al., 2000; Gordon \& French, 2005). In a study of immunocompetent adults aged from 18-64 years, over half of IPD was attributed to smoking, making 
smoking the strongest risk factor of those studied. There is a dose/response effect, with the strength of association between smoking and IPD increasing with an increase in the number of cigarettes smoked and the duration of the smoking history (Whitney, Schaffner, \& Butler, 2001). In addition to environmental risk factors, rare genetic mutations predispose some persons to IPD (Chapman et al., 2007; Hirschfeld et al., 2007; Ku et al., 2006).

Diagnosis of IPD

Diagnosis of IPD is dependent on the site of infection. For bacterial meningitis, most patients present with fever, headache, meningismus, and signs of cerebral dysfunction. Confirmation of this diagnosis is based upon the findings of an examination of cerebral-spinal fluid. Gram stain is a rapid method of identifying the pathogen, as is the employment of latex agglutination. Additionally, DNA amplification techniques such as Polymerase Chain Reaction (PCR) can be employed if the gram stain, bacterial antigen tests and cultures do not yield definitive results (Resti, Micheli, Moriondo, Becciolini, Cotimiglia et al., 2009; Smith et al., 2009; Tunkel \& Scheld, 2003; Verhelst, 2003).

Diagnosis of pneumococcal bacteremia is through focused physical examination resulting from a high index of clinical suspicion. Fever with chills, tachycardia, hypotension, and hyperventilation, often signs of infection, can point the clinician towards a diagnosis of sepsis and bacteremia. Confirmation of invasive pneumococcal sepsis or bacteremia is through blood cultures, with gram staining again as a tool for pathogen identification (Dorman, 2003). However, it has been noted that gram staining has greater utility in identifying $S$. pneumoniae in CSF than in other body fluids, and PCR might be the confirmation method of choice in these cases (Gray, 2000). In any 
case, to move a case from probable to confirmed IPD requires laboratory-confirmed culture of S. pneumoniae, in order to meet the CDC case definition and provide conclusive evidence of pneumococcal infection (CDC, 2007; Werno \& Murdoch, 2008).

Clinical classification of pneumococcal isolates is by capsular serotype (Austrian, 1999; Crook, 2006), though molecular genetic methods are sometimes employed, particularly in the classification of drug-resistant strains (Riley, 2004). Studies of colony morphology, sensitivity to optochin, and bile solubility also are employed in the clinical identification of pneumococci (Breiman et al., 2000; Rudolph, Parkinson, Reasonover, Parks, \& Butler, 2000; Verhelst et al., 2003). As an alternative to these methods, Multilocus Sequence Typing (MLST) or Polymerase Chain Reaction (PCR) may be used to determine the genotype of $S$. pneumoniae (Riley; Verhelst). Conventional culture methods are inexpensive and aid in determination of antibiotic resistance, however, culturing requires more time than MSLT or Polymer Chain Reaction (PCR) testing. Additionally, results of antibiotic resistance tests can be obscured if the IPD patient has already received antimicrobial therapy; genomic testing can identify resistant clones in the face of this previous antibiotic treatment (Abdeldaim, Stralin, Olcen, Blomberg, \& Herrmann, 2008).

Antibiotics in the Treatment of IPD

Inappropriate use of antibiotics has been put forward as the cause for geographic differences in $S$. pneumoniae drug resistance patterns, and as the cause of the development of drug-resistance on the whole (Hausdorff et al., 2000; McBean et al., 2005). Beta-lactam antibiotics have been the treatment of choice for IPD for over 50 years (Ball, 1999); however, treatment modalities frequently change. Changes in the 
treatment of IPD are driven by the emergence of drug-resistant serotypes (Maki, 2004), and by the development and acceptance of new antibiotics. As $S$. pneumoniae develops additional drug resistances, recommendations will continue to evolve.

Treatment failure has been documented in patients receiving ß-lactam antibiotic therapy for meningitis (Butler \& Cetron, 1999; Butler, Dowell, \& Breiman, 1998). Thus, for treatment of pneumococcal meningitis, the use of $ß$-lactams is inappropriate, and combination therapies with third-generation cephalosporins such as ceftriaxone or cefotaxime with vancomycin is indicated (with the vancomycin discontinued if the $S$. pneumoniae strain shows sensitivity to cefotaxime). Combination therapy is highly recommended, as the bacterium may develop resistance to ceftriaxone monotherapy (Ball, 1999; Greene and Demasi, 1996; Tunkel and Scheld, 2003). Levofloxacin, a fluoroquinolone, is also highly effective against $S$. pneumoniae (Goldstein \& GarabedianRuffalo, 2002; Tunkel \& Scheld, 2003).

In the treatment of bacteremia and pneumococcal sepsis, recommendations have included the use of macrolides or tetracyclines rather than $\beta$-lactams for treatment of IPD, though it must be noted that $S$. pneumoniae has shown some macrolide-resistance (Daneman, McGeer, Green, \& Low, 2006; Metlay et al., 2006). Other recommendations include the use of cephalosporins or a ß-lactam with a $ß$-lactamase inhibitor (such as piperacillin/tazobactam) combined with a macrolide or quinalone for treatment of the very ill (Ball, 1999). However, caution must be taken when inhibiting $\beta$-lactamase, as it appears that doses sufficient to inhibit mutated $S$. pneumoniae penicillin binding proteins PBP2x and PBP1a are also sufficient to induce tissue inflammation, with the potential for clinical complications (Moore et al., 2007). The use of vancomycin or a quinalone is 
essential when $\beta$-lactam resistance has been identified, with Linezolid or dalfopristin acceptable alternatives to vancomycin (in the absence of concurrent meningitis) (Dorman, 2003). Development of drug-resistance is a part of the survival strategy used by S. pneumoniae to avoid extinction (McBean et al., 2005), and the emergence and variation of drug-resistant clones may have an impact on epidemic dynamics. The Emergence and Impact of Drug-Resistant Streptococcus pneumoniae (DSRP)

Penicillin resistance is common in S. pneumoniae, as is resistance to other classes of antibiotics, particularly the cephalosporins (Rice, 2006). Outside of the previously noted optochin resistance in vitro, resistant pneumococci were first isolated in laboratory mice. However, drug-resistant strains in the human population did not emerge until the 1960’s (Butler \& Cetron, 1999). The first clinical reports of drug-resistant S. pneumoniae were from a single case in Australia in 1967 (Crewe-Brown et al., 1997), and a single pediatric case in a small village in New Guinea in 1969 (Tomasz, 1999). A case of drugresistant S. pneumoniae was reported in Chicago in 1969 (Parker, Davison, Paterson, \& Madden, 1970). More drug-resistance was identified in South Africa in 1973, then in Spain (the Spain 23F-1 and F-3 clones are now two of the most commonly encountered drug-resistant clones in the United States), and by the mid-1990's, drug-resistance was no longer rare in the United States (Bean \& Tomasz, 1973; Lawrenson et al., 1988)Richter et al., 2002; Tomasz (Klugman, 2003; L. McGee et al., 2001). The first drug-resistant strains in the United States emerged in the pediatric population, and the occurrence of drug-resistant strains remains highest in this subpopulation (Butler \& Schuchat, 1999). However, drug-resistant strains have become increasingly common in the subpopulation aged >65 years (Butler \& Cetron; Butler \& Schuchat). 
In the United States, outbreaks of drug-resistant $S$. pneumoniae have been reported in both childcare centers and long term care facilities. In some areas of the United States, 30\% of S. pneumoniae isolates exhibit antibiotic resistance (Hawley \& Whitney, 2002). By the mid-1990's, the prevalence of penicillin-intermediate and penicillin-resistant strains of S. pneumoniae reached $30 \%$ and $20 \%$, respectively (Ball, 1999). There is a significant association between recent treatment with ß-lactam antibiotics and new invasive infection with $\beta$-lactam resistant strains (Ball; Butler \& Cetron, 1999). Other predictors of infection with drug-resistant strains are attendance at day care centers and HIV-positive status (Ball, 1999). Both children and HIV-positive persons are vulnerable to acquisition of $S$. pneumoniae, including nosocomially. Nosocomial pneumococcal infections may contribute to as much as $40 \%$ of the cases of pneumococcal bacteremia (Bouza et al., 2005), and many of these hospital-acquired infections are drug-resistant (Crewe-Brown et al., 1997), possibly due to antibiotic selection pressures combined with poor host immunity.

Antibiotic selection pressure is an important factor in development of drugresistance $S$. pneumoniae. Selection pressure may result in the "un-masking" of drug resistant clones; e.g. the drug-resistant clones were present at lower and less likely to be detected levels than the non-resistant variants, and are revealed when the non-resistant colonization is eliminated. This then permits the amplification of the resistant clones. Selection pressure may also function by replacement, where the host ecology is cleared of non-resistant $S$. pneumoniae, and the drug-resistant strains replace them from community reservoirs of colonization (DeVecchi, Nicola, Ossola, \& Drago, 2009; Nuermberger \& Bishai, 2004). 
However, antibiotic and host immunity selection pressures, while important epigenetic events in the development of drug resistance (Trzcinksi et al., 2004), are not required for the acquisition of antibiotic resistance by $S$. pneumoniae. Clones of resistant strains can spread rapidly over large geographic areas. Interestingly, researchers have documented variation over time in emergence of virulent, resistant clones, with durations of emergent events of three and five years (Normark et al., 2001). These frequencydomain emergent events might function as attractors for epidemic events, events that may or may not present as predictable patterns in the long term.

Prevention of IPD

\section{Adult Pneumococcal Vaccines}

The current adult vaccine contains 25 micrograms of 23 purified capsular polysaccharide antigens (Butler, Shapiro, \& Carlone, 1999). The current adult vaccine covers approximately $90 \%$ of the serogroups that cause IPD in the United States. The effectiveness of both vaccines depends on the distribution of serotypes in the vaccinated population (Butler, Shapiro, \& Carlone; Kyaw, Clarke, Edwards, Jones, \& Campell, 2000).

The Advisory Committee on Immunization Practices recommends pneumococcal vaccination for those aged 65 and older, and those greater than two years of age at increased risk for IPD, with periodic revaccination recommended for certain subpopulations (Butler \& Cetron, 1999; Butler, Shapiro, \& Carlone, 1999; Mufson, 2000; Pletz, Maus, Krug, Welte, \& Lode, 2008). Consideration has been given to vaccinating the general population starting at age 50, particularly African Americans (Sisk, Whang, Butler, Sneller, \& Whitney, 2003). Additionally, HIV-positive persons with a CD4 count 
of 200 or greater should receive pneumococcal vaccination ( Breiman et al., 2000; Feikin et al., 2004; Penaranda et al., 2007).

Pneumococcal capsular polysaccharide vaccines create T cell-independent antibody responses, thus, they are not suited to children under two years of age (Pletz, Maus, Krug, Welte, \& Lode, 2008). This population appears to have some developmental limitations in T cell response, particularly in the neonatal subpopulation. This unsuitability is also true for some HIV-infected adults (Butler, Shapiro, \& Carlone, 1999; Chen et al. 1998; Feikin et al., 2004; Randolph \& Lewis, 2006; Sniadack et al., 1995). Pediatric Pneumococcal Vaccines

The current pediatric vaccine is a heptavalent conjugate formulation (the heptavalent pneumococcal conjugate vaccine, or PnCRM7) and addresses $70 \%$ of the serotypes most responsible for IPD in the pediatric population (Hausdorff, 2007). This vaccine is safe and efficacious, though significant differences in serotype-specific immune response has been reported (Kamboj, Kirchner, Kimmel, Greenspan, \& Schreiber, 2003). Reactions to the vaccine tend to be limited to localized swelling and redness, and did not increase with dose (Black \& Shinefield, 2002).

Conjugated vaccines may stimulate immunogenicity in the pediatric population, but are limited by a restricted number of antigens that may be included in a vaccine formulation (Butler \& Cetron, 1999). It is a conjugate vaccine that is recommended for use in the early pediatric subpopulation, and the vaccine has been found safe for simultaneous administration with the Hib vaccine. In fact, simultaneous vaccination with the pediatric conjugate vaccine and the Hib vaccine may be synergistic, improving the immunogenicity of the pneumococcal vaccine (Daum et al., 1997). Vaccination of infants 
with PnCRM7 has been shown to reduce $S$. pneumoniae carriage through at least three years of age (Millar et al., 2006). It is important to recognize that the heptavalent conjugate vaccine does not confer lifetime immunity. The eventual loss of immunity, coupled with the ease of transmission, is another factor that ensures the endemicity of $S$. pneumoniae infections (Keeling \& Rohani, 2008).

PnCRM7 confers protection by reducing colonization density, and this protection can occur as early as one month after the third priming dose, and may protect even earlier (O'Brien et al., 2007). Concerns have been raised, however, that the heptavalent vaccine does not cover Serotype 1, a serotype associated with a high attack rate and high morbidity. However, the study addressing this concern found a very low carriage rate of Serotype 1 S. pneumoniae (Brueggemann \& Spratt, 2003), and other study found that the incidence of serotype 1 had a high year-to-year variability (Hausdorff, 2007).

There are reports of non-vaccine types (NVTs) associated with IPD in the postvaccine era, and at least one of these reports refers to a fluctuating pattern in NVTassociated IPD incidence (Akduman, Ehret, \& Judson, 2006), reflecting the impact of NVT emergence on epidemic dynamics. Moore et al. (2008) and Pai et al. (2005) report on the emergence of serotype 19A some three to four years after implementation of the pediatric vaccine. Additionally, the strain reported in the Pai et al. study was antibiotic resistant and virulent, carrying the transposon-encoding for the Spain9v-3 clone, an internationally prevelant antibiotic-resistant variety (Pai et al., 2005; Pelton et al., 2007). Of note, the 19A serotype is addressed by the adult vaccine. Serotype 15 is another NVT identified as associated with IPD by the Pai et al. (2005) study, and has gained in prevalence in children hospitalized with IPD. Finally, the previously rare (in the United 
States and Europe) serotype 1, which is also an NVT, rapidly emerged (Normark et al., 2001). As NVTs increase in prevalence and acquisition of drug-resistance (Cao et al., 2009; Hanage et al., 2007; Lesley McGee, 2007), the patterns of epidemics of IPD could potentially change. Normark et al. suggest constant surveillance of serotypes to evaluate vaccine coverage as the NVTs replace vaccine serotypes as colonizers. Knowledge of the IPD epidemic pattern, if it exists, might aid in this surveillance.

Potentially impacting the epidemic system of IPD are pediatric vaccine shortages that took place in 2001 and in 2004 (Smith et al., 2007). Interruptions in measles vaccination altered the observed periodicity of that viral disease in the UK (Jansen et al., 2003). It is possible that any temporal variation that might exist in IPD could exhibit similar effects. While the long-term periodicity of IPD has yet to be established, thus the pressing need for this study, temporality and seasonality is characteristic of other human diseases.

\section{Temporality and Seasonality of Infectious Diseases}

Epidemics often display temporal variability, with variation in the causal agent, host responses, and transmission affecting this variability (Bansal et al., 2007; Grassly \& Fraser, 2006). Seasonal changes in disease incidence, that is, cyclic and predictable changes in disease patterns varying by the seasons of the year, have been identified for many diseases, and identification of epidemic patterns, including seasonality, has been a long-standing goal of mathematical epidemiology. Records dating back to the early $20^{\text {th }}$ century have been examined in attempts to find seasonal and periodic patterns for disease outbreaks, with some success (Bauch \& Earn, 2003). 
Among the diseases showing seasonality are influenza in temperate regions (Viboud et al., 2006), malaria following seasonal rain (Altizer et al., 2006), and IPD (Dowell et al., 2003). This seasonality can vary due to geography, changes in hostpathogen interactions, and due to changes in weather patterns. It is important from an epidemiologic standpoint to understand the seasonality of diseases, in order to target interventions, such as vaccination ahead of the influenza season, spraying for mosquitoes in the spring as a measure against the spread of West Nile virus (Altizer et al., 2006), and to detect changes in the epidemiology of the disease of interest (Finkenstadt \& Grenfell, 2000).

\section{Temporality and Seasonality of IPD}

IPD has a seasonal pattern of incidence; this pattern directly correlates to seasonal outbreaks of Respiratory Syncytial Virus (RSV) and Influenza, and with average hours of daily darkness, shorter daylight periods having higher incidence of IPD (Ampofo et al., 2008; Butler \& Schuchat, 1999; Kim et al., 1996; Stegemann et al., 2009; Talbot et al., 2005). When influenza and RSV outbreaks coincide, the incidence of IPD also significantly increases (Butler \& Schuchat; Jansen, Sanders, Van der Ende, Van Loon, Hoes, \& Hak, 2008; Talbot et al.). These influences on IPD seasonality leads to a peak incidence in the winter months (Dowell, Whitney, Wright, Rose, \& Schuccat, 2003; Kim et al.; Talbot et al.), with the exception of Alaska (Davidson et al., 1989). Within the broad area of epidemiologic inquiry, a small but important body of research has focused on a theory of seasonality, that is, the premise that $S$. pneumoniae outbreaks and epidemics correlate to recurrent weather patterns and temperatures. It has been shown that S. pneumoniae outbreaks occur during winter months (Dowell et al., 2003); the 
reason behind this seasonality remains unclear, with suggestions ranging from temperature influences to seasonal changes in host physiology (Dowell et al.; Dowell, 2001). In the study by Dowell et al. it was determined that temperature was not correlated with IPD, despite the clear seasonality of pneumococcal outbreaks. Weather can have an effect on infectious disease, with some organisms less viable at certain temperatures, and others taking advantage of changing host conditions due to weather. Additionally, other diseases with incidence fluctuation by season, specifically measles, have outbreaks that associate with the start of school years (Lloyd, 2001). Given the strong relationship between $S$. pneumoniae colonization and daycare or childcare center attendance (Huang, Finkelstein, \& Lipstich, 2005; Lencastre \& Tomasz, 2002), this might be a plausible cause for forcing of IPD seasonality.

\section{Periodicity of Diseases}

Seasonality, whatever the cause, might be viewed as a component of another larger phenomena ( Yulmetyev et al., 2003), that is, periodicity, the frequency-dependent recurrence of epidemics. Periodicity might also be described as a traveling wave phenomenon (Grenfell, Bjornstad, \& Kappy, 2001), relying for activation on bacterial colonization, playing against the attractors of new susceptibles, mutation of the bacterium, and both small and large networks of transmission, with inhibition by death or immunity (Grenfell, Bjornstad, \& Kappy; Kamo \& Sasaki, 2005). Periodicity of epidemics, with a regular frequency of occurrence, has been observed in several diseases.

Other diseases such as measles, mumps, rubella, RSV and varicella zoster (chicken pox) show a predictable recurrent pattern of epidemics and also show a seasonality comparable to that of IPD, particularly in the periods prior to the beginning of 
mass vaccination efforts (Duppenthaler, Gorgievski-Hrisoho, Frey, \& Aebi, 2003; Earn, Rohani, Bolker, \& Grenfell, 2000; Lofgren, Fefferman, Naumov, Gorski, \& Naumova, 2007). Measles, for instance, has demonstrated annual, biennial and triennial epidemic cycles, mumps a 3-5 year cycle, rubella, a 4-5 year cycle, RSV, a two year cycle, and varicella, cycles of 2-3 years (Anderson, Grenfell \& May, 1984). Of note, measles displayed periodic epidemics both before and after a vaccination effort (Grenfell et al., 2001; Riley, 2007). However, Ellner et al., (1998) and Olsen \& Schaffer (1990) argue that post-vaccination measles incidence reflects a chaotic system (one very sensitive to the effects of random events). If this is true, post-vaccination measles incidence should not have a detectable periodic pattern, as a chaotic system, by definition, is one that is in a state of disorder and irregularity (Yulmetyev, Yulmetyeva, \& Gafarov, 2005). Providing evidence contrary to these conclusions, Earn et al. (2000) and Grenfell et al. (2001) showed that the periodicity of measles epidemics is conserved, but with decreasing frequency and lower amplitude. This fits with the predictions made by Bolker \& Grenfell (1996), and helps to mitigate the earlier concern that reduced IPD incidence levels could prevent periodic pattern detection.

Infections due to $B$. pertussis exhibit a triennial cycle, post vaccination (Anderson, Grenfell, \& May, 1984). Pertussis was on an annual to a five year cycle in the pre-vaccine period, with little synchrony between geographic areas (Duncan, Duncan, \& Scott, 1996; Rohani, Earn, \& Grenfell, 1999). The effects of vaccination on pertussis periodicity, that is, a reduction in the force of transmission in the community, might have served to lengthen the inter-epidemic period, but the regularity of pertussis epidemics continue (Anderson et al., 1984), though the epidemic peaks are noisier (containing slight 
irregularities that do not detract from the overall frequency of occurrence) than those observed in measles, possibly due to stochastic recovery effects (Verdasca et al., 2005). Of note, different geographic areas fell into periodic synchrony in the post-vaccine period (Rohani et al., 1999). In examining the possible periodicity of IPD epidemics, it may be wise to evaluate the data in terms of geographic area. S. pneumoniae exhibits geographic heterogeneity in drug-resistance, and might exhibit similar characteristics in terms of epidemic pattern.

An epidemic of pertussis outside of the expected frequency led to identification of waning immunity in the adult population (Broutin, Guegan, Elguero, Simondon, \& Cazelles, 2005; Galanis et al., 2006; Guris et al., 1999; Oregon Department of Human Services, 2004). If $S$. pneumoniae has periodicity, this same warning mechanism might be employed. On the other hand, influenza, once thought to show an epidemic pattern of ten year intervals, has been shown to be aperiodic (Dowdle, 2006); this disallows the use of relative frequency for epidemic planning, forcing epidemiologists and public health planners to rely on techniques from weather forecasting in order to project influenza epidemics (Viboud, Boelle, Carrat, Valleron, \& Flahault, 2003). This technique should be employed, as the research reveals that $S$. pneumoniae outbreaks are aperiodic. Periodicity of IPD Epidemics

It was not known whether or not IPD epidemics occur in a periodic fashion, outside of our understanding of the seasonality of the disease. Several characteristics of IPD and $S$. pneumoniae created a plausible argument that such a pattern might exist. Among these are the microbial mechanisms that ensure endemicity and prevent extinction, the distribution of $S$. pneumoniae colonization, which relies on children in 
crowded settings as a reservoir; this reservoir will undergo periodic replenishment with a new group of susceptibles, and the observed periodicity in serotype 1 colonization. However, no study analyzing IPD incidence rates over time to establish periodicity in the United States or any other location could be found, and this is the gap in the literature that the study fills. The following section reviews the data sets required to provide this information.

\section{Models based on Time-Series Analysis}

The noise, specifically observational noise (Chen \& Bokka, 2005; Keeling \& Rohani, 2008b), exhibited in an epidemic system can be difficult to separate from chaos (Stewart, 2002). To avoid this confusion, awareness of random perturbations over time (stochasticity) (Stewart, 2002), hidden Markov chains (the results of stochastic processes that may result in a "settling" of the pattern and thus may obscure periodicity) (Meyn \& Tweedie, 1993), and their potential to affect the results of a study is important (Francq \& Roussignol, 1997; Olsen \& Schaffer, 1990), and drives model selection to a great degree. Rohani et al. (1999) concluded that pertussis, when examined from a deterministic perspective, is very sensitive to stochasticity, which should be taken into account when selecting a model for analysis. It is not known whether IPD also exhibits this sensitivity, but when selecting a model and technique for elucidating IPD epidemic dynamics, the potential for sensitivity to stochastic effects must be considered.

When cyclic epidemics are more regular in occurrence than would be expect edbased on chance, an appropriate model can be fitted, and later used in surveillance (Hamaker, Dolan, \& Molenaar, 2005). One set of models suitable for this purpose are those based in the repeated measurement of a set of variables, the multivariate time 
series, or time-series analysis (Hamaker, Dolan, \& Molenaar). Models built on timeseries analysis can be based on the time domain, or based on frequency domain analysis, specifically, the Fourier Transform (a mathematical approach for transforming a wave process to a simpler linear expression) (Wei, 1994b), which produces a periodogram (a visual representation of the quantitative results of the Fourier Transforms which facilitates the analysis of a frequency domain time-series) (Wei, 1994a) that correlates to the contribution of the varied frequency components to the observed patterns of disease incidence (Anderson et al., 1984; Lindsey, 2004a).

When selecting the appropriate model to represent epidemic dynamics, it is essential to ask several questions. First, what is the role of the initial conditions, that is, the number of susceptible persons, the index case, the rate of infectivity, the number of persons exposed, and the virulence of the pathogen at the start of the very first outbreak? (Keeling \& Rohani, 2008b). In modeling IPD, the initial conditions are unknown. Secondly, have observations, such as the count of cases of IPD, been made regularly or irregularly in terms of time? The source of data for this study, the National Hospital Discharge Survey, is based on regular observations. Third, is the dynamic of the epidemic stationary, that is, does the incidence increase or decrease systematically over time without peaks? Epidemics exhibit non-stationarity (Keeling \& Rohani). Finally, does the epidemic dynamic appear to follow a Markov process, that is, the current condition of disease incidence is dependent on the condition occurring immediately before it in time? Answering these questions will aid in selecting the appropriate model of epidemic dynamics (Lindsey, 2004b). 
Deterministic Models

Deterministic time series models have been used in resolving the underlying processes of disease transmission and incidence (Lloyd, 2004). However, stochastic events can cause fluctuations in disease patterns, and deterministic models are not accurate in the setting of random perturbations (Lloyd, 2004). Because of the unpredictable and labile nature of the $S$. pneumoniae organism, with its ready sharing of genetic materials and agility in evading the host immune response, the resultant incidence of IPD may well occur in a stochastic, non-Markov fashion, ruling out the use of a deterministic model that might exhibit complex and unrealistic patterns (Lloyd, 2001). Furthermore, a deterministic model requires an initial condition, and seasonal forcing must be accounted for (Earn et al., 2000).

For measles, the initial condition used in building a deterministic model was the transmission process, that is, the path from the index case to the next group of infected persons (Bjornstad, Finkenstadt, \& Grenfell, 2002). However, if a simulation is run against a deterministic model, for each run with the same initial condition, the results must be the same, in a clockwork process, in other words, an epidemic dynamic that appears to occur as if by the mechanism of a gear-driven machine, with no variation or deviation from the predicted path (Bjornstad, Finkenstadt, \& Grenfell). Epidemics do not truly function in clockwork, mechanistic fashion, and the randomness of the epidemic process once it begins presents difficulty in modeling (Yulmetyev et al., 2003). It is hard to imagine an epidemic that would occur in the exact same fashion if repeated, with the same people acquiring the disease each time. For measles to truly model in a deterministic fashion, the initial conditions must be known, and the epidemic must follow 
the same path for each realization from the same ensemble. Therefore, deterministic models may not be ideally suited to the description of epidemic phenomena, or for identifying periodicity of epidemics, the goal of the research.

\section{Stochastic Models}

This leads towards discussion of a non-deterministic, stochastic model as a more appropriate approach for the study. Mollison, Isham, \& Grenfell (1994) observe that for large populations, deterministic models can approximate epidemic behaviors, but these models can only approximate the mean behavior of the system (Finkenstadt \& Grenfell, 2000). Stochastic modeling of epidemics can be difficult, because chance events might be major factors in the eventual size and timing of an epidemic. Still, stochastic models can more accurately describe epidemic patterns (Finkenstadt, Bjornstad \& Grenfell, 2002; Mollison et al., 1994). The primary advantage to such a model is the ease by which they can be built, by virtue of familiar mathematics (Mollison et al.).

However, a stochastic model can aid in understanding the process of the epidemic, and while more complicated mathematically (Mollison et al., 1994), is better suited to determination of an epidemic pattern. It must be acknowledged that a stochastic model could exhibit disease fadeout (the extinction of a disease expressed in a mathematical model, most likely as a result of a sufficient drop in the number of infected and susceptible individuals in a given population within the model) ( Keeling \& Rohani, 2008b; Lloyd, 2004), but this is unlikely in the case of IPD, given the ubiquity of $S$. pneumoniae colonization, the emergence of NVT serotypes, and the other factors that mediate in favor of IPD endemicity. Some evidence has been gathered from animal models showing that epidemic periodicity may be determined in part by the introduction 
of new susceptibles and the acquisition of immunity (Anderson et al., 1984). Underlying this conclusion is a theory of the mass action principle of transmission, that is, the incidence of a disease is dependent on the density of colonized or infectious individuals, multiplied by the density of susceptible persons (Anderson et al.; Grassley \& Fraser, 2006; Wilson \& Worcester, 1945). According to Grassly and Fraser (2006), if an infection results in either immunity or death, the law of mass action predicts disease cycles. Seasonal variations due to deviations in the pattern of disease transmission will occur due to inconsistent contact patterns (and thus a variable contact rate) (Soper, 1929). This might account for disease cycles in infectious diseases that do not confer immunity. In addition to these seasonal variations in contact patterns, replenishment of susceptible hosts, colonization with $S$. pneumoniae, and occurrence of IPD can be reasonably expected to occur in a random fashion and vary over time, and health policy changes, vaccination campaigns and vaccine shortages are external factors that could act as attractors in a stochastic system and influence the dynamics of the system (Xia, Bjornstad, \& Grenfell, 2004), introducing a degree of uncertainty (Koelle \& Pascual, 2004), or pushing the system into a stable cycle (Earn et al., 2000). To further complicate model building, irregularities in vaccine supply, as happened with the pediatric vaccine against $S$. pneumoniae, and variations in vaccine effectiveness, such as from low efficacy vaccines or serotype replacement, will account for changes to epidemic patterns (Alexander, Moghadas, Rohani, \& Summers, 2006) in a stochastic manner. As shown in work on the dynamics of measles epidemics, for utility in epidemiology, any potential model must be able to account for this stochasticity (Grenfell, Bjornstad, \& Finkenstadt, 2002; Xia et al., 2004). 
Yulmetyev et al. (2003) have demonstrated the use of the Fast Fourier Transform (FFT) for the purpose of detecting periodicity in epidemics, describing constancy between the epidemic periods of Acute Respiratory Tract Infections (ARTI) with the causal organisms unidentified. This particular work demonstrated the successful use of the FFT, and by extension, frequency-domain analysis in a setting of a stochastic, nonMarkov epidemic process, of which IPD arguably fits. The use of a stochastic, nonMarkov process for analysis of epidemic patterns takes into account the dynamic nature and non-stationarity of epidemic systems, yielding a more accurate and detailed view of the epidemic course (Mollison et al., 1994; Yulmetyev et al.).

Modeling of Disease Dynamics using Time-Series Approaches while Accounting for Stochasticity

The literature contains several notable examples of the application of time series approaches to the determination of epidemic dynamics. Many of these studies also approached the problem from a stochastic perspective. Measles, rotavirus, and pertussis have all been studied in this fashion.

A study of infection dynamics that employed mathematical methods to account for stochasticity and chaos focused on rotavirus. Several models were used to examine rotavirus dynamics, both deterministic and non-deterministic. Each model revealed a different aspect of the population dynamic (Jose \& Bishop, 2003). After an examination of the results, Jose and Bishop determined that the dynamic was best described by a variation of time-series analysis, wavelet-based incidence analysis. The results of wavelet analysis revealed an annual, biannual, and triennial period, as well as other embedded cycles. While the patterns exhibited self-similarity over multiple scales (a fractal pattern), 
the existence of a periodic and predictable epidemic pattern could not be determined. A chaotic dynamic, as found in this study of rotavirus infection dynamics, is well suited to the wavelet analysis technique (Cazelles, Chavez, Berteaux, Menard, Vik, Jenouvrier, et al., 2008).

This method of time-series analysis had been previously applied to measles population dynamics, which have a known periodicity (Grenfell, Bjornstad, \& Kappy, 2001). Grenfell, Bjornstad and Kappey (2001) applied a wavelet approach to the analysis, as opposed to traditional Fourier analysis. While Grenfell, Bjornstadt and Kappy stated that this method has potential as a tool for analysis, they noted that the wavelet method requires a large set of data. Most significantly, they observed that the deterministic model they developed using this analysis makes predictions that did not match reality (Grenfell, Bjornstad \& Kappey, 2001). Wavelet analysis techniques may have value in understanding aperiodic epidemics (Cazelles et al., 2008). Because S. pneumoniae epidemics have a known periodic component, seasonality, analyses based on wavelet approaches are not indicated, and are not recommended for the study.

Another approach to understanding epidemic dynamics using time-series analysis has been the modification of existing deterministic models to incorporate time series methods. An example of this modification is an analysis of whooping cough epidemics in London, Duncan, Duncan and Scott (1996) employed a modification of the SusceptibleInfected-Recovered (SIR) model through application of time-series analysis. This modification yielded a model that described the changing epidemic dynamic of whooping cough during the period from 1701 to 1812 in London. The model was then compared to a time-series analysis of wheat prices for the same time period, and the correlation 
between wheat prices (as an marker of nutrition in the population) and whooping cough was examined. This study illustrates the value of understanding the multiple year epidemic dynamics. Once the pattern was discerned, it was used to help identify potential causal factors, and in this case, elucidated the role of malnutrition in whooping cough outbreaks (Duncan, Duncan \& Scott, 1996).

Stone, Olinky, and Huppert (2007) also modified the forced SIR model, by applying frequency domain time-series analysis to measles data from the US and the UK, and using the results to modify the SIR model. Seasonal forcing was removed from the model, and the dynamic of the multiple year epidemic cycle was substituted. This facilitated a better understanding of post-epidemic dynamics, and provided a model better suited to prediction of future outbreaks (Stone, Olinky \& Huppert, 2007).

This modification of the SIR model to examine measles epidemic patterns led to extension of the modified model, the TSIR (Time series SIR) model (Bjornstad, Finkenstaedt \& Grenfell, 2002) to further account for stochasticity. The TSIR model was described as a discrete time stochastic analogue of the SIR model (Grenfell, Bjornstad \& Findkenstaedt, 2002). By including consideration of spatial transmission patterns, Xia, Bjornstad and Grenfell (2004) introduced additional stochastic elements into the model. The consideration of stochastic factors led to a model with a better fit with historical measles dynamics (Xia, Bjornstad, \& Grenfell, 2004).

Previous Attempts at Constructing a Model of Sepsis Epidemic Patterns using TimeSeries Analysis

Sepsis is a component of IPD, and a review of the literature revealed one study that attempted to employ time-series analysis to determine the epidemic patterns of 
sepsis. An investigation of all-cause sepsis periodicity showed an increase in sepsis incidence over time; this study employed a deterministic model (Danai, Sinha, Moss, Haber, \& Martin, 2007). This research revealed that sepsis increased in incidence over the study period. However, during the same period, vaccination appears to have reduced the incidence of IPD (Hammitt et al., 2006; Jackson \& Janoff, 2008;McBean, Park, Caldwell, \& Yu, 2005; Moore et al., 2004; Poehling et al., 2006; Shah \& Ratner, 2005). The observed rise in sepsis in light of an observed fall in IPD incidence over the same period suggests that the Danai et al. study should not be used to describe IPD epidemic patterns, and the increase in sepsis incidence described in this study might be reflective of possible increases in other pathogen incidence. Danai et al. used a deterministic model that, while mathematically less complex than a stochastic model, might not have been ideal for examination of epidemic dynamics (Mollison et al., 1994; Yulmetyev et al.). No other articles could be found in the literature attempting to examine IPD periodicity. A Model of IPD Dynamics Amenable to Modification as a Temporally-Forced Model using Time-Series Analysis

The SIR model has been modified to account for stochasticity and incorporate time series analysis (Keeling \& Rohani, 2008c). This model is suitable for infectious diseases in which immunity is acquired by surviving the infection. IPD does not present a good fit to the SIR model, because not all susceptible and exposed persons become infected and infection with $S$. pneumoniae does not confer permanent immunity (BridyPappas, Margolis, Center \& Isaacman, 2005; Millar et al., 2008). Therefore, a better model for describing the epidemic dynamics of IPD might be the Susceptible-ExposedInfected-Recovered (SEIR) model, which can account for the variance between exposed, 
infected and infectious states (D'Innocenzo, Paladini, \& Renna, 2006; Keeling \& Rohani, 2008b). Sutton, Banks \& Castillo-Chavez (2008) have applied this model to IPD, with the inclusion of seasonality to result in a Temporally Forced Model. Colonization rates serve as a proxy for exposure, and birth rates, death rates, and vaccination rates are factored into the model, as is seasonality (Sutton, Banks, \& Castillo-Chavez, 2008). The data used to calculate rates and modify model parameters was either obtained from existing literature or direct measurement. This resulted in a deterministic model that was employed to assess the impact of a recently implemented pneumococcal vaccination program. The initial conditions of the deterministic model were derived from historical data. The model was calibrated by comparison to existing data, and required only minor modification of parameters to obtain a fit (Sutton, Banks, \& Castillo-Chavez, 2009). This model of IPD epidemic dynamics provides a starting point for further model development. It does, however, exhibit an important weakness.

Beyond the weakness inherent in the deterministic nature of this model, a weakness of this model is that it does not account for any variation in the epidemic pattern beyond a seasonal increase. Thus, it is only capable of modeling a single epidemic, in a continuous dynamic. Modification of this model, to account for stochastic effects from periodicity, might create a model of greater accuracy and utility. This modification requires knowledge of the IPD epidemic pattern beyond one year. This information would then be used to alter the transfer rate equations in the model to reflect a cyclical increase or decrease at a set frequency.

Any modification of the SEIR model must be contemplated in light of the observations of Finkenstaedt and Grenfell (2000). Establishing a connection between the 
time series and the SEIR model could be problematic because the only directly observable variable is the number of infected individuals, and this might be underreported. Parameters such as birthrates and vaccination rates change over time, and this could impact the accuracy of the model. Finally, the SEIR model is a continuous dynamical system, and time-series analysis in the frequency domain is based upon discrete time intervals (Finkenstaedt and Grenfell, 2000).

A modification to the Sutton, Banks, and Castillo-Chavez model of IPD dynamics (2008) would share some of those limitations, but not all. Under reporting is a consideration, but the introduced noise would only lower the amplitude of observed periods. By use of frequency domain rather than time domain techniques, the patterns, if any, to IPD epidemic dynamics would still be revealed. The SEIR model of IPD dynamics can be linked together as sequential realizations, with the rate of entry into the population adjusted between each annual realization to account for varying birthrates, either historical or predicted, dependant on the purpose of the modeling. The existing model already provides the opportunity to input the vaccination rate (Sutton, Banks, \& Castillo-Chavez, 2008), which again could be modified for each realization. Realizing the entire model as a sequential linkage of individual models ameliorates the concern over use of discrete time data in a continuous dynamical system. Each annual model would retain its continuous dynamic, with only that year's incidence and seasonal forcing applied. Thus, an individual year is a continuous model, but the whole is constructed on discrete intervals.

The SEIR model has been realized as just such a discrete-interval stochastic model. Using measles, as described in the work of Anderson and May (1991), Verdasca, 
da Gama, Nunes, Bernardino, Pacheco, and Gomez (2005) assumed differing amplitudes of seasonal forcing, and also realized the model in an unforced configuration. They acknowledged the need to modify the models parameters for vaccination and other variables. Their results supported the conclusion that the model must take these factors into account, but also that the resulting model can fit readily to the observed data. They also observed that this model is computationally feasible (Verdasca, da Gama, Nunes, Bernardino, Pacheco, \& Gomez, 2005).

\section{A Recommended Approach for Description of Epidemic Patterns of IPD}

IPD displays cyclic epidemics that are regular in occurrence, by seasonality (Dowell, Whitney, Wright, Rose, \& Schuchatt, 2003). In situations where cyclic epidemics are more regular in occurrence than would be expected based on chance, models can be devised and later used in surveillance (Hamaker, Dolan, \& Molenaar, 2005). Appropriate to such situations are those models built upon on the repeated measurement of a set of variables (Hamaker, Dolan, \& Molenaar). Models built on these repeated measurements of discrete variables can be based on either the time domain or frequency domain (Wei, 1994b). As it is the frequency of IPD epidemics that is a focus of this research, time-series analysis in the frequency domain is the most suitable approach.

In recommending this approach, consideration was given to the role of initial conditions, that is, the number of susceptible persons, the index case, the rate of infectivity, the number of persons exposed, and the virulence of the pathogen at the start of the very first outbreak (Keeling \& Rohani, 2008b). In IPD, the initial conditions are unknown. That the initial conditions are unknown is a key reason for selection of a stochastic approach for this study, rather than a deterministic approach. For IPD to 
accurately model in a deterministic fashion, the initial conditions must be known, and the epidemic must follow the same path for each realization from the same ensemble (Bjornstad, Finkenstadt, \& Grenfell, 2002). Therefore, a deterministic model is not ideally suited to the description of IPD epidemic periodicity.

Also, consideration is given to the regularity or irregularity of observations of a key variable, the count of cases of IPD. Regular observations are a component of this study's source of data, the National Hospital Discharge Survey. Finally, stationarity of the epidemic dynamic and the possibility of a Markov process are considered. Epidemics exhibit non-stationarity (Keeling \& Rohani), and no evidence could be found in the literature that IPD epidemics follow a Markov process. These considerations weigh on the selection of the appropriate approach, and lead to the exclusion of time-series analysis focused on deterministic systems (Wei, 1994e).

\section{Stochastic Approaches}

An analytic approach that takes stochastic behavior and focuses on the frequency component of the epidemic dynamic is more suitable to this study. It must be acknowledged that a stochastic model could lead to results that suggest disease fadeout (the extinction of a disease expressed in a mathematical model, most likely as a result of a sufficient drop in the number of infected and susceptible individuals in a given population within the model) ( Keeling \& Rohani, 2008b; Lloyd, 2004), but this is unlikely in the case of IPD, given the ubiquity of S. pneumoniae colonization, the emergence of NVT serotypes, and the other factors that mediate in favor of IPD endemicity. Further aiding in the argument in favor of an approach that accounts for stochasticity is the random nature of replenishment of susceptible hosts, colonization with 
S. pneumoniae, and seasonal variation in IPD incidence. External factors such as irregularities in vaccine supply or serotype replacement, as noted in the review of the literature, could influence the dynamics of the system ( Alexander, Moghadas, Rohani, \& Summers, 2006; Xia, Bjornstad, \& Grenfell, 2004), introducing stochasticity (Koelle \& Pascual, 2004), or pushing the system into a stable cycle (Earn et al., 2000). As shown in work on the dynamics of measles epidemics, the approach must be one that is capable of dealing with this potential stochasticity (Grenfell, Bjornstad, \& Finkenstadt, 2002; Xia et al., 2004). An approach that is non-deterministic and focused on the detection of periodic events is time-series analysis in the frequency domain, by use of the Fast Fourier Transform (FFT).

Yulmetyev et al. (2003) have demonstrated the successful use of the FFT, and by extension, frequency domain analysis in a setting of a stochastic, non-Markov epidemic process, which describes IPD epidemics. The use of a stochastic, non-Markov process for analysis of epidemic patterns takes into account the dynamic nature and non-stationarity of epidemic systems, yielding a more accurate and detailed view of the epidemic course (Mollison et al., 1994; Yulmetyev et al.). The Fast Fourier Transform produces a periodogram, a visual representation of the quantitative results of the transform, which facilitates the analysis of a frequency domain time series (Wei, 1994a) that correlates to the contribution of the varied frequency components to the observed patterns of disease incidence (Anderson et al., 1984; Lindsey, 2004a).

The results of such an analysis can then be applied to the SEIR model put forth by Sutton, Banks and Castillo-Chavez (2008). By applying the periodic component as a factor in the existing models transfer rate equation to reflect a cyclical increase or 
decrease in disease incidence at the dominant frequency, the model could better fit the IPD disease dynamic by reflecting the effects of time on multiple epidemic occurrences. By using predicted birth rates, death rates, and the periodic component, the modified model could be used as a predictive tool. When IPD dynamics differ from the predictive model, the situation could then be examined for changes in IPD epidemiology. However, as the results revealed that IPD does not have a periodic epidemic dynamic, so the SEIR model remains as currently envisioned by Sutton, Banks, and Castillo-Chavez.

Given a technique for construction of the model, and an appropriate framework for model selection, the size and quality of the data set must be addressed, as in order to join a model with the observations, both large and full data sets are needed (Lindsey, 2004a; Lloyd, 2001). The study used the National Hospital Discharge Survey (NHDS) as the data set. This is both a large and full data set, as the following review will illustrate.

National Hospital Discharge Survey (NHDS) as a Secondary Data Source for the Research

\section{Definition and History of the NHDS}

The NHDS is a continuous national probability survey, intended to gather and disseminate information on the inpatient utilization of non-Federal short stay hospitals (Dennison \& Pokras, 2000; National Center for Health Statistics, 2007a). The data within the NHDS is derived from the medical records of inpatients discharged from a national sample of approximately 500 hospitals (Boulet et al., 2006). The NHDS is considered to be the principle source of national level data on the characteristics of patients discharged from these hospitals (Lou \& Zack, 2005). 
The NHDS has operated continually since its inception in 1965 , but some revisions and updates have been needed throughout the life of the system. The largest change occurred in 1988, when the NCHS undertook a redesign of the survey to link the survey to the National Health Interview Survey (NHIS) and to permit a wider selection of statistical software to be brought to bear on the data. Additionally, the coding methods and data file variables have evolved and changed over the history of the survey (Dennison \& Pokras, 2000), specifically the transition from the International Classification of Diseases, $8^{\text {th }}$ Revision (ICD-8) to the International Classification of Diseases, $9^{\text {th }}$ Revision, Clinical Modification (ICD-9CM). The transition from the ICD-8 to ICD-9CM in 1978 involved a change in the codes assigned to individual diseases, and thus to the variables contained in the NHDS files. The reporting of the diseases themselves were not affected, only the code assigned to them (Dennison \& Pokras).

Data collection and sampling procedures have undergone modification, as well. Beginning in 1985, two data collection procedures have been used in the survey, a manual system from the hospital records to abstract forms, performed by hospital staff, and an automated system where data is purchased from a variety of commercial vendors, hospitals, and public agencies (National Center for Health Statistics, 2007c). Approximately 40 percent of information is obtained through the automated system (Dennison \& Pokras, 2000). From the 1988 revision to date, the sampling plan is a threelevel stratified design, with all hospitals of 1,000 beds or greater included in the sample, and smaller hospitals included as samples within the other two strata (Dennison \& Pokras). 
NHDS Data Sources and Contributors

The data sources and contributors to the NHDS have evolved along with the survey itself. In the beginning of the survey, the sample was selected from short-stay hospitals listed in a National Master Facility Inventory, with the sample stratified by hospital size (bed count), and geographic region. After the 1998 redesign, the sample was selected from a listing of eligible hospitals in the Specialty Medical Group (SMG) Hospital Market Database. This sampling scheme was used until 2003, when the listing changed to the Healthcare Market Index and Hospital Market Profiling Solution. Both of these listings were formerly components of the SMG Hospital Market Database, thus the data source remains essentially the same, with only the name of the database changed and the main source of the data split into two reports (Hospital Care Statistics Branch, 2007).

As it has done throughout its history, the NHDS currently covers discharges from non-institutional hospitals. Federal, military, and Veterans Administration hospitals are excluded from the survey. Additionally, patients in the sampled hospitals must have an average length of stay of less than 30 days, and the hospital must have a declared specialty of general medical or general surgical, or a children's general specialty to be included in the survey. Finally, surveyed hospitals must have six or more beds staffed for patient use (Hospital Care Statistics Branch, 2007).

When sampling this population of hospitals, large hospitals (1000 beds and greater), and those with the highest number of discharges (compared among the reporting hospitals, and varying from year to year) are always selected, and the remainder of the sample is selected according to a three-stage sampling scheme (Dennison \& Pokras, 2000). Hospitals are stratified by whether or not they subscribe to a commercial 
abstracting service, and by size. Subsequent samples are based upon the type of service provided by the hospital and by bed count. The sample frame is updated every three years to allow for changes in hospital size or services and to capture hospitals that opened after the last sampling (National Center for Health Statistics, 2007b). The information obtained through these sampling frames is organized into a defined structure, enabling the efficient handling and use of the data.

\section{The Structure of NHDS Data}

The design of the NHDS is focused on deriving accurate estimates of inpatient hospital utilization. SUDAAN, a statistical software package for analysis of data from complex studies, is used to calculate the standard errors in these estimates, and all statistics used in these calculations are made available to users of the data (Kozak, Defrances, \& Hall, 2006). This transparency is a hallmark of the NHDS. This is only a general look at NHDS data structure, and a further exploration of how the NHDS structures the large volume of information it gathers is in order.

The NHDS performs data gathering, manipulation, and distribution in accordance with three prioritized objectives. The first priority objective is national aggregate statistics, the second is national trend statistics, and the third is aggregate statistics for the four major United States Census regions (Dennison \& Pokras, 2000). This prioritization of effort occurred as part of the 1988 redesign of the NHDS, and informs the basic structure of the NHDS.

As have the sampling frames, NHDS variables have evolved throughout the life of the survey. These variables are provided as part of the documentation included with each NHDS annual file. A review of the list of data variables reveals a variable set that 
appears to be comprehensive and relevant to the declared purpose of the database, and pertinent to the declared objective of the NHDS. These variables are appropriate for this study. The use of specific disease codes will enable the identification of IPD cases from within the large data set. The current iteration of the NHDS relies on the use of ICD-9CM coding as the key variable for disease identification.

The Use of ICD-9CM Coding as a Key Variable in the NHDS, and Quality Assurance for Coding and Data Accuracy.

The NHDS has made use of standardized disease codes from its inception, starting with the ICD-8. As the coding standard changed, so did the variable within the NHDS. Currently, the NHDS expresses diagnostic information at discharge using ICD9CM codes, inclusive of all ages, genders, and geographic location. For data received on manual worksheets, the coding process is validated by sampling ten percent of each batch of 1000 records. These samples are coded by a second coder, and any discrepancies are resolved by a chief coder. New coders processing NHDS data have 100 percent of their first three batches audited, and half of their remaining work for the first year is also audited. Any batch that has an error rate greater than five percent must be completely recoded. When data entry and quality control is completed, a file of records is sent to NCHS for editing, estimation and further processing.

For the automated data, the quality control process is different. First, the electronic files are evaluated for physical integrity. If the file is unusable, a new one is requested. Files are then brought into a standard format and layout. All of the records are then evaluated for missing or inaccurate information, and the results are reviewed for invalid ICD-9CM codes. If any problems identified can't be resolved by NCHS, a new 
file is requested, and the process begins again upon receipt of the new file (McLemore \& Pokras, 2001).

For the most part, coding errors are the greatest hazard to reliability of the data within the NHDS (Popovic, 2001). Hospital discharge data has been considered by some to be more accurate and reliable than that obtained by surveys, as the information on diagnosis is obtained directly from the health care providers (De Bruin, van Heel, Lukens, \& Hoes, 2005; Machlin, Cohen \& Thorpe, 2000). Recall bias on the part of the patient is thus less of a concern (Machlin, Cohen \& Thorpe). Therefore, within reason and given an understanding of the methodology of the survey, users of NHDS data may accept its reliability.

Correlation of NHDS Data to Population Estimates is Essential to Calculating Incidence

Conveniently, the NHDS data provided on CD-ROM is already correlated to population estimates, with included calculated error rates, so that researchers can readily use it to determine incidence rates. NHDS data obtained from on-line sources must be utilized with the included United States Census files to correlate to population efforts (National Center for Health Statistics, 2007b). This requires analysts to ensure that the correct census data is used when computing population rates; the NHDS provides the appropriate census files as part of the on-line file with the annual utilization data.

Once decoded, the NHDS data lends itself to analysis and manipulation with many available programs and systems. It remains that the format is not immediately importable to many of these systems, and the use of the CDC Statistical Export and Tabulation System (SETS) is recommended for initial conversion of the NHDS data to the proper format if the commercial Statistical Analysis Software (SAS) is not the 
statistical analysis program of choice. It must be further noted that personal information and subject identifiers are not retrievable from the NHDS data set, regardless of software, as the NHDS is designed with robust privacy protections.

Privacy Protections in the NHDS.

The original data source for the NHDS is not available to the public (Lou \& Zack, 2005), and in the original data source, names or other subject unique identifiers are not obtained from the reporting hospitals (Popovic, 2001). Additionally, the publicly available files do not include medical record numbers, date of birth, admission and discharge dates, in fact, all direct identifiers, are omitted from the data set (Popovic). These restrictions and omissions from the public data set provide adequate privacy protections, and bring the NHDS into compliance with the Health Insurance Portability and Accountability Act (HIPAA), as well as the appropriate sections of the Public Health Service Act (Popovic). This protection of subject privacy is a strength of the NHDS; other strengths and limitations require further discussion.

Strengths and Limitations of the NHDS

Conveniently, the NHDS data provided on CD-ROM is already correlated to population estimates, with included calculated error rates, so that researchers can readily use it to determine incidence rates (National Center for Health Statistics, 2007b). This counts as a strength of the NHDS. However, the transmittal of this data by File Transfer Protocol, either in ASCII or SETS formats requires either statistical software with specific input files, such as SAS, or other, intermediate software, such as SETS or a text editor that will accept ASCII. This requires users of the data to obtain this software, and is a limitation of the NHDS when used in an on-line environment. 
The NHDS makes use of ICD-9CM codes to express discharge diagnosis, as do many compilations of data used in health services (De Coster et al., 2006), and this use of a standardized coding format can be considered one of the strengths of the NHDS. Still, users of NHDS data must stay aware of the fact that the use of ICD-9CM coding for tracking disease and mortality data is widely practiced, but has some limitations (Kloss, 2005). Early studies of the NHDS concluded that demographic information in the database was highly reliable, and that discrepancies in this data component were centered on dates of admission and discharge, date of birth or age, and gender. It was further concluded that these errors were largely caused by problems in transcription.

However, these researchers also found that the reliability of diagnostic information varied widely, depending on the coding scheme in use (Demlo \& Campbell, 1981). The reliance of the NHDS on ICD-8, and later ICD-9CM, codes for classifying discharge diagnosis means that the quality of the data set is greatly dependent on the accuracy of coding (Guevara et al., 1999). Because the NHDS is a major source of national level data for hospital discharge information, these questions of reliability must be considered. The accuracy of the ICD-9CM codes entered into the NHDS data base is verified by the CDC (Popovic, 2001). Therefore, to reduce the impact of reliability issues, the study used data gathered and coded after 1978 and the transition to the ICD9CM coding scheme.

Furthermore, the utility of these codes in studies of specific disease entities, for example, pneumococcal incidence and cardiac arrest, has been upheld by independent study (De Bruin, van Hemel, Leufkens, \& Hoes, 2005; Guevara et al., 1999). Nonetheless, other studies have reported underestimation of incidence in the NHDS 
(Boulet, Correa-Villansenor, Hsia, \& Atrash, 2006), and researchers must remain aware of reliability issues within the NHDS related to coding. Other limitations in the NHDS are related to the completeness of data, specifically data on race and ethnicity.

Data on race and ethnicity within the NHDS is often missing (Dennison \& Pokras, 2000), or underreported (Kozak, 1995). This is not isolated to the NHDS, as researchers have noted information on race and ethnicity either missing or unreliably reported in other hospital discharge surveys and databases. This missing data may be attributed to the fact that many hospitals are not mandated to gather this information. As of 2005, only 22 states required hospital collection of this information, and even then, the information was not always collected (Schoenman et al., 2005). Under-reporting of data will not impact the possible periodicity of IPD; rather, it will lower the amplitude of any detected periodic events (Keeling \& Rohani, 2008b). Thus, the missing data does not significantly impact the study.

Researchers examining the under-reporting of race within the NHDS are clear in their recommendations. They clearly advise against attempting proportional adjustments of NHDS data, as it would possibly correct the estimate of some discharges, while overestimating others (Kozak, 1995). The study goes on to state that "no ideal solution exists" to the problem, and that NHDS race data be used with caution (Kozak). As to the category changes in the 2000 census, Parker et al. (2004) recommend the use of bridge models to ensure comparability between older data and current data. These recommendations may or may not be suitable, and users of NHDS data may want to exclude the use of race or ethnicity in analysis, or pursue other databases with more reliable data. Again, the under-reporting of race in the NHDS simply inserts a degree of 
observational noise into the model, and should have no effect on the possible phenomenon of periodicity of IPD epidemics (Keeling \& Rohani, 2008b).

A Serious Gap in Our Knowledge of IPD Epidemic Dynamics

From the preceding review, the depth and breadth of our understanding of the epidemiology of IPD can be discerned. Detailed understanding of the microbial mechanisms of colonization and infection, host vulnerabilities and factors contributing to colonization and infection, and the seasonal nature of IPD is apparent. Knowledge of these factors is essential to attaining an understanding of their interplay in potential epidemic dynamics. Mechanisms ensuring microbial survival also ensure endemicity, which influences incidence rates, and the patterns of epidemics. Changes in host vulnerability, from vaccination or lack of vaccination, and effective or ineffective use of antibiotics, can also provide plausible reasons for variations in IPD epidemic patterns.

This review of the literature also reveals established concepts and tools for analysis of data related to IPD; specifically, the use of time-series analysis to elucidate patterns of epidemics. Choices exist in models, and the choice made clearly depends on the nature of the question and ease of computation. Finally, the existence of an archival data set conducive to the study has been reviewed.

A large and detailed body of knowledge exists for addressing IPD, epidemic seasonality, and epidemic modeling. However, a serious gap existed in this body of knowledge, in that the epidemic periodicity of IPD has not been determined. It is this gap that this study addresses. Through the use of time-series analysis applied to the NHDS data set, this study determined that a pattern of IPD epidemics did not exist, and described a chaotic epidemic dynamic. The following chapter will address specifics of 
the study design, sample, and analytic techniques used to answer the important question: Does invasive infection by S. pneumoniae in the United States occur in a periodic epidemic pattern of a predictable recurrent nature and definable frequency? 


\section{CHAPTER 3: METHODS}

\section{Introduction}

In the previous chapter, current literature addressing the epidemiology of IPD was reviewed, with emphasis on mechanisms that might influence potential epidemic dynamics. The review of the literature also examined approaches to identification and analysis of epidemic periodicity; specifically, the use of frequency-domain Time Series Analysis to establish epidemic dynamics was reviewed. Lastly, the characteristics of an archival data set conducive to this study were discussed. Clearly, a large and detailed body of knowledge exists addressing IPD, epidemic seasonality, and epidemic modeling. However, a serious gap exists in the literature covering IPD; the existence or absence of epidemic periodicity of IPD has not been determined. This study is intended to fill this gap, and the discussion in this chapter will address specifics of the study design, sample, and analytic techniques intended to address this gap in the literature.

\section{Study Design and Approach}

The general intent of this study was to provide a detailed description of the epidemic pattern of invasive disease caused by $S$. pneumoniae infection by relating incidence to time for a cohort of discharged hospitalized patients in the United States with a discharge diagnosis of IPD, taken from the National Hospital Discharge Summary (NHDS) database for the period of 1979-2006. In order to develop this detailed description, the study utilized a retrospective cohort study design. To accomplish the objective of the study, the endemic level of IPD in the United States during the period from 1979-2006 was determined, as well as the incidence level of IPD in the United 
States during the same period. This provided information essential to answering the research questions:

(1) Do epidemics of IPD occur in a periodic manner with a definable frequency in addition to the predicted seasonal increase in incidence, in the aggregate, in the United States? Do epidemics of IPD occur in a periodic manner with a definable frequency in addition to the predicted seasonal increase in incidence, by gender, in the United States? Do epidemics of IPD occur in a periodic manner with a definable frequency in addition to the predicted seasonal increase in incidence, by age (in ranges), in the United States? And Do epidemics of IPD occur in a periodic manner with a definable frequency in addition to the predicted seasonal increase in incidence, by geographic area, in the United States

The hypotheses were as follows: $\mathrm{H}_{\mathrm{o} 1}$ : IPD did not occur in periodic epidemics of a definable frequency outside of the predicted seasonal increase in incidence in the United States for the period from 1979-2006, in the aggregate. $\mathrm{H}_{\mathrm{a} 1}$ : IPD occurred in periodic epidemics of a definable frequency in addition to the predicted seasonal increase in incidence in the United States during the period from 1979-2006, in the aggregate. 
(3) $\mathrm{H}_{\mathrm{o} 2}$ : IPD did not occur in periodic epidemics of a definable frequency outside of the predicted seasonal increase in incidence in the United States for the period from 1979-2006, by gender. $\mathrm{H}_{\mathrm{a} 2}$ : IPD occurred in periodic epidemics of a definable frequency in addition to the predicted seasonal increase in incidence in the United States during the period from 1979-2006, by gender. $\mathrm{H}_{\mathrm{o}}$ : IPD did not occur in periodic epidemics of a definable frequency outside of the predicted seasonal increase in incidence in the United States for the period from 1979-2006, by age (in ranges). $\mathrm{H}_{\mathrm{a} 3}$ : IPD occurred in periodic epidemics of a definable frequency in addition to the predicted seasonal increase in incidence in the United States during the period from 1979-2006, by age (in ranges). $\mathrm{H}_{\mathrm{o} 4}$ : IPD did not occur in periodic epidemics of a definable frequency outside of the predicted seasonal increase in incidence in the United States for the period from 1979-2006, by geographic area. $\mathrm{H}_{\mathrm{a} 4}$ : IPD occurred in periodic epidemics of a definable frequency in addition to the predicted seasonal increase in incidence in the United States during the period from 1979-2006, by geographic area.

Calculation of endemicity and incidence rates occurred prior to the time-series analysis of the data. Calculation of the cumulative incidence was made by dividing the number of IPD cases during the period from 1979-2006 by the size of the population at the start of each of year within the period from 1979-2006 totaled. The US census information, provided for each year of the NHDS data, was used to establish the 
denominator. The resulting information was used to establish the cumulative rate of incidence of IPD per 100,000 population from 1979-2006, and the incidence rates of IPD per 100,000 population stratified by gender, age in ranges and by geographic area. The mean of each these incidences was calculated to establish the endemic levels of IPD for the period 1979-2006. The endemic level was calculated to enable establishment of epidemic levels in the presence of periodic outbreaks; periodicity was not observed, thus this level was not used in subsequent analyses.

The question of epidemic recurrence at definable frequency was then addressed by frequency domain time-series analysis. Frequency domain time-series analysis was achieved through the employment of the SPSS 17.0 for Mac statistical software package; the Forecasting module command set Spectra was applied to the data for the transform, analysis, and construction of the periodogram (SPSS, Chicago). Visual display of quantitative data is through the use of graphs developed using SPSS 17.0 for Mac. This analysis yielded information on perturbations in the epidemiologic pattern over time (when compared to the normal disease pattern). These perturbations, if present, would constitute evidence of large epidemics of invasive disease, and if present can be examined and analyzed to establish the existence of epidemic periodicity (and the frequency of that periodicity) or aperiodicity.

\section{Setting and Sample}

The study is archival research; the data for the study is contained in the CDC's National Hospital Discharge Survey (NHDS) database (Popovic, 2001), for the period ranging from 1979 to the most recent year of compiled data, 2006. These data sets are publicly accessible, by either purchase from the National Technical Information Service, 
or by download from the CDC server. For the data sets from 1979 to 1995, a multi-year CD-ROM was purchased from the National Technical Information Service, (order number PB99-501876). The data for the period 1996 to 2006 was downloaded by File Transfer Protocol (FTP) from the CDC server at http://nber15.nber.org/nhds/ftp.cdc.gov/ pub/Health_Statistics/NCHS/Datasets/NHDS/. Documentation for the on-line data sets was also obtained by FTP from the CDC server at http://nber15.nber.org/nhds/ ftp.cdc.gov/pub/Health_Statistics/NCHS/Dataset_Documentation/NHDS.

The NHDS, the major source of national level aggregative information on hospital utilization in the United States, is a continuous national probability survey targeting the inpatient utilization of non-Federal short stay hospitals, through the gathering and processing of data derived from the medical records of inpatients discharged from a national sample of approximately 500 hospitals (Dennison \& Pokras, 2000; National Center for Health Statistics, 2007a). The data within the NHDS are derived from the medical records of inpatients discharged from a national sample of approximately 500 non-institutional hospitals (Boulet et al., 2006). The NHDS is considered to be the principle source of national level data on the characteristics of patients discharged from these hospitals (Lou \& Zack, 2005).

Federal, military, and Veterans Administration hospitals are excluded from the NHDS. Additionally, hospitals must have an average in-patient length of stay of less than 30 days, a declared specialty of general medical or general surgical, or a children's general specialty to be included in the survey. Finally, surveyed hospitals must have six or more beds staffed for patient use. The NHDS expresses diagnostic information at 
discharge using ICD-9CM codes, inclusive of all ages, genders, geographic location, and co-morbidities (Hospital Care Statistics Branch, 2007).

\section{Description of the Study Population}

The population for the study was defined as those cases with a discharge diagnosis of IPD included in the NHDS data base (Popovic, 2001), for the defined time period of 1979-2006. The accuracy of the ICD-9CM codes entered is verified by the CDC (Popovic), and the utility of these codes in studies of pneumococcal incidence has been upheld by independent study (Guevara et al., 1999). Subject selection criteria will be set narrowly, by selecting specifically those conforming to the ICD-9CM codes consistent with IPD. The NHDS data set is searchable for these codes, as well as those for age, gender, race, geographic region, and dates of admission and discharge. The use of ICD-9CM coding to select a sample has been employed an earlier significant study that addressed seasonality of IPD, but not periodicity (Dowell et al., 2003). To further clarify the study population, definitions of IPD are in order, moving from a conceptual definition, through a case definition, and finally to an operational definition.

\section{Case Definition}

IPD was defined as a clinically compatible case, with a laboratory-confirmed culture of $S$. pneumoniae isolates obtained from blood, cerebral spinal fluid (CSF), pleural fluid, pericardial fluid, joint aspirate, or other normally sterile sites. This definition specifically excluded otitis media, as the specific causal organism is rarely identified for this diagnosis and it rarely requires in-patient treatment. Furthermore, infections with other Streptococcus species, such as S. pyogenes, S. scarlatinae or S. puerperalis were excluded from this definition of IPD. These organisms, while clinically 
important in their own right, have different clinical character than S. pneumoniae (Breiman et al., 2000).

\section{Operational Definition of IPD}

For an operational definition of IPD, the research study used specific International Classification of Diseases, $9^{\text {th }}$ Revision, Clinical Modification (ICD-9CM) codes consistent with three general diagnostic groupings of IPD: septicemia, bacteremia, and meningitis. These codes are pneumococcal septicemia (038.2), bacteremia (790.7) with an accompanying pneumococcus (041.2), pneumococcal meningitis (320.1), or any combination thereof.

\section{Sampling Method and Frame}

The sample design employed in the study was multi-stage random sampling (Trochim, 2001), with elements of both probability and non-probability sampling. The initial sampling was conducted in the process of compiling the NHDS, which is a probability sample representing a sample of hospitals, as discussed earlier. The next stage of sampling was the identification and extraction of the subpopulation of NHDS-listed IPD cases by specific ICD-9CM codes for analysis, which is a non-probability sample.

The sampling frame of the NHDS is a continuous national probability survey of a national sample of approximately 500 hospitals. In compiling the NHDS, large hospitals (1000 beds and greater), and those with the highest number of discharges (compared among the reporting hospitals, and varying from year to year) are always selected, and the remainder of the sample is selected according to a three-stage sampling scheme (Dennison \& Pokras, 2000). Hospitals are stratified by whether or not they subscribe to a commercial abstracting service, and by size. Subsequent samples are based upon the type 
of service provided by the hospital and by bed count. The sample frame is updated every three years to allow for changes in hospital size or services and to capture hospitals that opened after the last sampling (National Center for Health Statistics, 2007b). The sampling frame of the NHDS was thus confind to large hospitals, specifically, those with the highest count of discharges are selected with certainty. The remainder of the sample was selected according to a three-stage plan.

The first group selected as a result of the three-stage plan is a sample from geographic region. Within those groupings, hospitals are further stratified by whether or not they subscribe to a commercial abstracting service, and by size. The third stage samples are based upon the type of service provided by the hospital and by bed count. The sample frame is updated every three years to allow for changes in hospital size or services and to capture hospitals that opened after the last sampling (National Center for Health Statistics, 2007b).

The next stage of sampling for the study occurred with the selection of IPD cases from the NHDS data set for the period from 1979, when the use of ICD-9CM codes was instituted (Popovic, 2001) to 2006 (the last year for which data have been compiled). Within this data set, all cases of IPD will be selected. This sample frame is necessary to conduct an accurate frequency-domain time-series analysis; a total count of events at each point in time (monthly for the performed analysis) is used to establish the components of the spectrum (Wei, 1994). Time-series analysis differs from descriptive statistics in that omission of cases by random sampling might distort the analysis, by reducing the amplitude of any periodic components and thus potentially reducing the 
amplitude of any events below the level needed to distinguish between periodicity and chaos (Yulmetyev, Yulmetyeva, \& Gafarov, 2005).

Thus, the first stage of sampling was a probability sample (by the design of the NHDS), and the second stage was non-probability sampling that (driven by the requirements of frequency-domain time-series analysis) included all IPD cases compiled from the dataset. Eligibility for inclusion in the study sample was determined by ICD9CM code.

\section{Eligibility Criteria}

Subject selection criteria was set narrowly, by selecting specifically for pneumococcal septicemia $($ ICD-9CM code $=038.2)$ or bacteremia (ICD-9CM code $=$ 790.7) with an accompanying pneumococcus (ICD-9CM code $=041.2$ ), as well as for pneumococcal meningitis (ICD-9CM code $=320.1)$, or any combination thereof. All ages, genders, races, and geographic regions as defined in the NHDS data set were included. Non-specific codes for septicemia (ICD-9CM code $=038)$, bacteremia (ICD9CM code $=790.7)$ or bacterial meningitis $($ ICD-9CM code $=320)$ were excluded from the analysis, to avoid gathering data on invasive disease that might have been attributable to other pathogens, such as Staphylococcus aureus, Moraxella catarrhalis, or Klebsiella pneumoniae. Additionally excluded were cases of otitis media, as the specific causal organism is rarely identified, nor does it often require in-patient treatment (Breiman et al., 2000). Furthermore, infections with other Streptococcus species, such as S. pyogenes, S. scarlatinae or S. puerperalis were excluded from case selection. 
Instrumentation and Materials

Having defined the setting and sample for the study, and discussed the sampling frame and methods, the focus now turns to the instrumentation and materials required to conduct this study. In the ensuing discussion, a description of the dataset, data collection tools, processes, and the location of the raw data will be covered.

Study Instruments, Type of Instrument, and Concepts Measured by the Instruments

The study dataset for the research consisted of the individual data sets from the NHDS from 1979-2006. These files are organized by individual year, and the format varies depending upon the year of publication. For the period from 1979 to 1996, a multiyear CD-ROM provides all of the applicable files (National Center for Health Statistics, 2007a). The data set for this date range in the study was the CD-ROM, National Technical Information Service (NTIS) order number PB99-501876. For the years 1996 to 2006, the data sets for the study were obtained from a CDC server through FTP via compressed file sets that contain data elements in ASCII (American Standard Code for Information Interchange) format (CDC, 2007), an American National Standards Institute (ANSI) standard format that permits interoperability across a variety of platforms (American National Standards Institute, 2002). Documentation files unique to each year were transmitted or stored on the CD-ROM uncompressed, either in Adobe Portable Document Format (.pdf) or Text format (.txt), again dependant on the year that the data were published. The actual format of data in the NHDS is determined by the syntax laid out in the survey codebook. The codebook for the NHDS is published annually as part of the supporting documentation for the annual survey, and is contained as an addendum to the documentation file. Each individual record within the NHDS data is listed as a 
separate line consisting of numeric string, with values and positions in the numeric string corresponding to those listed in the codebook (National Center for Health Statistics, 2007b).

A Detailed Description of the Variables Contained in the Data

The variables contained in the numeric string are listed in the documentation included with each NHDS annual file. The variables in the NHDS case string are as follows:

(1) Survey Year (the last two digits of the survey year)

(2) Newborn status (coded as $1=$ newborn, 2 = not newborn)

(3) Units for age (coded as $1=$ years, $2=$ months, $3=$ days)

(4) Age in years, months, or days (coded as units = years, 00-99, If units $=$ months, 01-11, If units-days, 00-28). Fields for years, months and days exist in the dataset.

(5) Sex (coded as $1=$ male, $2=$ female $)$

(6) $\quad$ Race $($ coded as $1=$ White, $2=$ black, $3=$ American Indian/Alaskan Native, 4 = Asian, 5 = Native Hawaiian or other Pacific Islander, $6=$ Other, $8=$ Multiple race indicated, $9=$ Not stated)

(7) Marital status (coded as $1=$ Married, $2=$ Single, $3=$ Widowed, 4 $=$ Divorced, $5=$ Separated, $9=$ Not stated)

(8) Discharge month (coded 01-12 = January to December)

(9) Discharge status (coded 1 = Routine/discharged home, $2=$ Left against medical advice, 3 = Discharged/transferred to short-term 
facility, 4 = Discharged/transferred to long-term care institution, 5

$=$ Alive, disposition not stated, $6=$ dead, $9=$ Not stated or not reported)

(10) Days of care (coded as the actual number of days)

(11) Length of stay flag ( $\operatorname{coded} 0=$ Less than 1 day, $1=$ One day or more)

(12) Geographic region ( coded $1=$ Northeast, $2=$ Midwest, $3=$ South, $4=$ West $)$

(13) Number of beds, recode (coded $1=6-99,2=100-199,3=200$ $299,4=300-499,5=500$ and over)

(14) Hospital ownership (coded $1=$ Proprietary, $2=$ Government, $3=$ Nonprofit, including church)

(15) Analysis weight (used to obtain weighted estimates)

(16) First two digits of survey year

(17-23) Diagnosis codes (1970-77: one to five 4-digit ICD-8 diagnostic codes: 1979-2006; one to seven 5-digit ICD-9-CM codes)

(24-27) Procedure codes (1970-77: zero to three 3-digit ICD-8 procedure codes: 1979-2006: zero to four 4-digit ICD-9-CM procedure codes)

(28) Principal expected source of payment (not contained in the data set for 1977-97) (coded as $1=$ Worker's compensation, 2 = Medicare, $3=$ Medicaid, 4 = Other government, $5=$ Blue Cross/Blue Shield, 
$6=\mathrm{HMO} / \mathrm{PPO}, 7=$ Other private insurance, $8=$ Self-pay, $9=$ No charge, $10=$ Other, $99=$ Not stated)

(29) Secondary expected source of payment (not contained in the data set for 1977-97) (coded as $1=$ Worker's compensation, $2=$ Medicare, 3 = Medicaid, $4=$ Other government, $5=$ Blue Cross/Blue Shield, $6=\mathrm{HMO} / \mathrm{PPO}, 7=$ Other private insurance, 8 $=$ Self-pay, $9=$ No charge, $10=$ Other, $99=$ Not stated)

(30) Diagnosis-related group (DRG) codes: 1986-2006 (Not on multiyear file)

(31) Type of Admission (coded as $1=$ Emergency, $2=$ Urgent, $3=$ Elective, 4 = Newborn, $9=$ Not available)

(32) Source of Admission (coded as 1 = Physician referral, $2=$ Clinical referral, $3=$ HMO referral, $4=$ Transfer from a hospital, $5=$ Transfer from a skilled nursing facility, $6=$ Transfer from other health facility, $7=$ Emergency room, $8=$ Court/law enforcement, 9 $=$ Other, $99=$ Not available) (National Center for Health Statistics, 2007).

The geographic regions for the NHDS are as follows:

(1) Northeast: Maine, New Hampshire, Vermont, Massachusetts, Connecticut, Rhode Island, New York, New Jersey, Pennsylvania

(2) Midwest: Michigan, Ohio, Illinois, Indiana, Wisconsin, Minnesota, Iowa, Missouri, North Dakota, South Dakota, Nebraska, Kansas 
(3) South: Delaware, Maryland, District of Columbia, Virginia, West

Virginia, North Carolina, South Carolina, Georgia, Florida, Kentucky, Tennessee, Alabama, Mississippi

(4) West: Montana, Idaho, Wyoming, Colorado, New Mexico, Arizona, Utah, Nevada, Washington, Oregon, California, Hawaii, Alaska (Hospital Care Statistics Branch, 2007).

The NHDS data provided on CD-ROM is already correlated to population estimates, with included calculated error rates, so that researchers can readily use it to determine incidence rates. NHDS data obtained from on-line sources must be utilized with the included United States Census files to correlate to population efforts (National Center for Health Statistics, 2007a). This required assurance that the correct census data were used when computing population rates; the NHDS provides the appropriate census files as part of the on-line file with the annual utilization data.

\section{Processes for Assessment of the Reliability and Validity of the Data}

A key assumption of the study was the reliability and validity of the data from the NHDS database. The NHDS makes use of ICD-9CM codes to express discharge diagnosis, as do many compilations of data used in health services (De Coster et al., 2006), and this use of a standardized coding format can be considered one of the strengths of the NHDS. Still, users of NHDS data must stay aware that, although the ICD-9CM coding system for tracking disease and mortality data is widely practiced, the approach has some limitations (Kloss, 2005). Early studies of the NHDS concluded that demographic information in the database was highly reliable, and that discrepancies in this data component were centered on dates of admission and discharge, date of birth or 
age, and gender. Kloss further concluded that these errors were largely caused by problems in transcription.

However, these researchers also found that the reliability of diagnostic information varied widely, depending on the coding scheme in use (Demlo \& Campbell, 1981). The reliance of the NHDS on ICD-9CM codes for classifying discharge diagnosis means that the quality of the data set is greatly dependent on the accuracy of coding. Significantly, the utility of these codes in studies of specific disease entities, and of particular importance to the study, pneumococcal incidence, has been upheld by independent research (Guevara et al., 1999).

To ensure accurate data, the NHDS employs quality control techniques dependant on the submitted format. For the most part, coding errors are the greatest hazard to reliability of the data within the NHDS (Popovic, 2001), and error detection procedures are in place to ensure that these hazards are reduced. For potential NHDS data received on manual worksheets, the coding process is validated by sampling ten percent of each batch of 1000 records. These samples are again coded by a second coder, and any discrepancies are resolved by a chief coder. New coders processing NHDS data have $100 \%$ of their first three batches audited, and half of their remaining work for the first year is also audited. Any batch that has an error rate greater than five percent must be completely recoded. When data entry and quality control is completed, a file of records is sent to NCHS for editing, estimation, and further processing (McLemore \& Pokras, 2001).

For the automated data, the quality control process is different. First, the electronic files are evaluated for physical integrity. If the file is unusable, a new one is 
requested. Files are then brought into a standard format and layout. All of the records are then evaluated for missing or inaccurate information, and the results are reviewed for invalid ICD-9CM codes. If any problems identified can't be resolved, a new file is requested, and the process begins again upon receipt of the new file (McLemore \& Pokras, 2001).

Hospital discharge data has been considered by some to be more accurate and reliable than that obtained by surveys, as the information on diagnosis is obtained directly from the health care providers. Recall bias on the part of the patient is thus less of a concern (Machlin, Cohen \& Thorpe, 2000). However, an issue of data accuracy relates to analysis of the data by race or ethnicity. Data on race and ethnicity within the NHDS is often missing (Dennison \& Pokras, 2000), or underreported (Kozak, 1995). This missing data may be attributed to the fact that many hospitals are not mandated to gather this information. As of 2005, only 22 states required hospital collection of this information, and often, the information was not always collected (Schoenman et al., 2005). Thus, the research excluded the use of race or ethnicity in analysis.

Based upon the quality assurance measures in place, the accuracy of the NHDS data may be assumed. The NHDS data set is sufficiently large and detailed to permit frequency domain time-series analysis. Additional discussion of the NHDS, NHDS sampling frames and techniques, and studies addressing the accuracy of the NHDS may be found in Chapter Two.

\section{Location of the Raw Data}

The raw data for the study was obtained from two sources. In the case of the multi-year CD-ROM, the data set was purchased from the National Technical 
Information Service, as order number PB2005-500003, available on-line at http://www.ntis.gov/search/product.aspx?ABBR=PB2005500003. The data for the period 1996 to 2006 was downloaded by FTP from the CDC server at http://nber15.nber.org/nhds/ftp.cdc.gov/pub/Health_Statistics/NCHS/Datasets/NHDS/. Documentation for the on-line data sets was downloaded by FTP from the CDC server at http://nber15.nber.org/nhds/ftp.cdc.gov/pub/Health_Statistics/NCHS/Dataset_Documenta tion/NHDS. Given the extremely large number of cases catalogued in 28 years of hospital discharges, it is impractical to include the raw data in the appendix of this paper.

\section{Data Collection and Analysis}

Data sets were obtained from either the CD-ROM (for years 1979-2001) or the on-line files (for the years 2001- 2006). Data extraction was conducted through the use of the TextEdit for Mac (OS X 10.5.5) application (Apple, Cupertino, CA). Once the data for each year was extracted into .txt format, it was read into SPSS 17.0 for Mac (SPSS, Chicago), and each annual file was converted to SPSS .sav format. This process was repeated for each year's data set, and duplicate data sets were compared to each other to ensure accurate transfer and formatting.

Files with errors during comparison were deleted, and the process repeated, to ensure the accuracy of data. After error checking was complete, each annual file was saved as an independent file, backups of each individual file were made, and the backups stored at an off-site location. Finally, the annual files were merged into a cumulative file using the SPSS 17.0 for Mac Merge Files Add Cases command; the resulting file was saved in SPSS .sav format, and backed up as with the individual annual files.

The first analysis was focused on determining the endemic level of IPD in the 
United States for the period from 1979-2006, in the aggregate, and by gender, age (in ranges), and geographic area. Using the SPSS Data Select command on the merged data set, IPD cases by ICD-9CM code (NHDS variables 17-23), specifically pneumococcal septicemia (038.2) or bacteremia (790.7) with an accompanying pneumococcus (041.2), as well as for pneumococcal meningitis (320.1), were selected and the resulting cases counted by use of the SPSS 17.0 for Mac Analyze/Descriptive Statistics/Frequencies command. Calculation of the cumulative incidence for the period from 1979-2006 followed. The formula for this calculation was: Number of IPD cases during the period from 1979-2006/Size of the population at the start of each of year within the period from 1979-2006 totaled. The US census information provided for each year of the NHDS data was used to establish the denominator.

This process was repeated with the data further selected by gender (NHDS variable 5), then repeated with the data further selected by age (in ranges) (NHDS variable 4 , recoded as $1=0-4$ years, $2=5-14$ years, $3=15-24$ years, $4=25-34$ years, 5 $=35-44$ years, $6=45-54$ years, $7=55-64$ years, $8=65-74$ years, $9=75-84,10=85-98)$, and finally repeated with the data further selected by geographic area (NHDS variable 12). The resulting information was used to establish the cumulative rate of incidence of IPD per 100,000 population from 1979-2006, and the incidence rates of IPD per 100,000 population stratified by gender, age in ranges and by geographic area. The mean of each these incidences was calculated to establish the endemic levels of IPD for the period 1979-2006.

The next phase of analysis was targeted to determine the predicted incidence level of IPD during seasonal increases, and to answer the research questions: 
(1) Do epidemics of IPD occur in a periodic manner with a definable frequency in addition to the predicted seasonal increase in incidence, in the aggregate, in the United States?

(2) Do epidemics of IPD occur in a periodic manner with a definable frequency in addition to the predicted seasonal increase in incidence, by gender, in the United States?

(3) Do epidemics of IPD occur in a periodic manner with a definable frequency in addition to the predicted seasonal increase in incidence, by age (in ranges), in the United States? And

(4) Do epidemics of IPD occur in a periodic manner with a definable frequency in addition to the predicted seasonal increase in incidence, by geographic area, in the United States?

Using the appropriate SPSS Data Sort and Select commands on the merged data set, IPD cases by the specific ICD-9CM code (NHDS variables 17-23) for IPD were selected. The results were then be subjected to frequency-domain time-series analysis through the employment of the SPSS 17.0 for Mac add-on module Forecasting. The SPSS 17.0 for Mac Forecasting command set Spectra was used to execute an FFT and plot a periodogram of IPD incidence for each month in the period from 1979-2006.

Additional periodograms were derived from the data, in the aggregate and stratified by gender, age in ranges, and geographic region for each month in the period from 1979-2006. These periodograms were examined with attention to detection of periodic components in addition to the expected seasonal incidence. These perturbations were examined and analyzed to establish or refute the existence of epidemic periodicity 
(and the frequency of that periodicity), addressing the study research question. All analytic results were reported in both numeric and graphic formats. Graphics were generated by the SPSS 17.0 for Mac software package. All data, raw, reformatted, and analyzed, had personal identifiers removed as part of the NHDS data set development process.

Analysis of the data facilitated the calculation of the cumulative incidence level of IPD in the United States for the period from 1979-2006, in the aggregate, and by gender, age (in ranges), and geographic area. Using the SPSS Data Select command on the merged data set, IPD cases by ICD-9CM code (NHDS variables 17-23), specifically pneumococcal septicemia (038.2) or bacteremia (790.7) with an accompanying pneumococcus (041.2), as well as for pneumococcal meningitis (320.1), were selected and the resulting cases counted by use of the SPSS 17.0 for Mac Analyze/Descriptive Statistics/Frequencies command. Annual incidence rates were then calculated, using the formula: Estimated number of IPD cases for each year/Size of the US population for that year. Calculation of the cumulative incidence of IPD in the United States for the period of 1979-2006 followed. The formula for this calculation was: Number of IPD cases during the period from 1979-2006/Size of the US population for each of year within the period from 1979-2006 totaled. The US census population information provided for each year of the NHDS data was used to establish the denominator. The results are expressed as cases per 100,000 .

This process was repeated with the data further selected by gender (NHDS variable 5), then repeated with the data further selected by age (in ranges) (NHDS variable 4 , recoded as $1=0-4$ years, $2=5-14$ years, $3=15-24$ years, $4=25-34$ years, 5 
$=35-44$ years, $6=45-54$ years, $7=55-64$ years, $8=65-74$ years, $9=75-84,10=85-98)$, and finally repeated with the data further selected by geographic area (NHDS variable 12). The results are expressed in terms of cases per 100,000 . The outcomes of the first analysis were then used to calculate the endemic rate of IPD per 100,000 from 19792006. As previously noted, the endemic level was calculated to enable establishment of epidemic levels in the presence of periodic components to the IPD dynamics; if these had been detected, the level of these components would have been compared to the endemic level in order to establish or refute the presence of an epidemic. Periodicity was not observed in this study, thus this level was not used in subsequent analyses.

The third phase of the analysis concentrated on refuting or establishing the existence of periodic epidemics of IPD, beyond the previously established seasonality. Using the appropriate SPSS Data Sort and Select commands on the merged data set, IPD cases by the previously identified ICD-9CM codes (NHDS variables 17-23) were selected. The results were then subjected to frequency-domain time-series analysis through the employment of the SPSS 17.0 for Mac add-on module Forecasting. The SPSS 17.0 for Mac Forecasting command set Spectra was used to execute an FFT and plot periodograms of IPD incidence for the period from 1979-2006. These periodograms were generated for the all variables data set, and for data stratified by gender, age in ranges, and geographic region. The periodograms were generated in the frequency domain (Wei, 1994f).

These periodograms were examined and analyzed to establish or refute the existence of epidemic periodicity (and the frequency of that periodicity) using the techniques elucidated by Wei (1994f). This analysis addressed the hypotheses of this 
study. The results of this analysis were expressed in graphic form, using the graphics generated by the SPSS 17.0 for Mac software package.

An additional analysis was conducted to indentify seasonality of IPD in the United States for the period from 1979-2006. As in the previous analysis, the SPSS 17.0 for Mac Forecasting command set Spectra was used to execute a FFT based on a modified Cooley-Tukey algorithm (SPSS, 2008) and plotted a periodogram of IPD cases by month for the period from 1979-2006. As in the prior analysis, these periodograms were generated in the frequency domain (Wei, 1994f). The data was not analyzed by strata of time period, as the analytic techniques used require long time series, and stratification into shorter groups of years would not have met this requirement (Komalapriya et al., 2008)

\section{Measures for the Protection of Participant Rights}

Walden University Institutional Review Board (IRB) approval was granted for this study, under IRB approval number 05-28-09-0328781. The original data source for the NHDS is not available to the public (Lou \& Zack, 2005), and in the original data source, names or other subject unique identifiers are not obtained from the reporting hospitals (Popovic, 2001). Additionally, the publicly available files do not include medical record numbers, date of birth, admission and discharge dates, in fact, all direct identifiers, are omitted from the data set (Popovic). These restrictions and omissions from the public data set ensure privacy protection, and bring the NHDS into compliance with the Health Insurance Portability and Accountability Act (HIPAA), as well as the appropriate sections of the Public Health Service Act (Popovic, 2001). The Public Health Service Act states that data collected by the National Center for Health Statistics can only 
be used for health reporting and analysis, and prohibits any other use or attempts to determine the identity of a case (National Center for Health Statistics, 2007). 


\title{
CHAPTER 4: RESULTS
}

\author{
Introduction
}

The purpose of this study was to describe the epidemic pattern of IPD in the United States in the last 28 years. The following research questions were addressed:

(1) Do epidemics of IPD occur in a periodic manner with a definable frequency in addition to the predicted seasonal increase in incidence,
(a) in the aggregate,
(b) by gender,
(c) by age range, and
(d) by geographic area.

Two specific objectives were addressed in order to answer these questions. First, the incidence levels of IPD for each year and for the combined period in the time span from 1979-2006 were estimated. Calculation of incidence levels permitted the comparison of the results of this study to those of previous studies, and acted as a check against intrusion of non-pneumococcal disease into the analysis, which would have been reflected in incidence levels that rose, rather than fell after the introduction of the pediatric vaccine. Second, the endemic levels of IPD in the United States were calculated for the period from 1979-2006 in the aggregate, and by gender, age (in ranges), and geographic area. The endemic level was calculated to enable establishment of epidemic levels in the presence of periodic outbreaks; periodicity was not observed, thus this level was not used in subsequent analyses. These subsequent analyses used the estimated count of cases within each month of the time period to construct periodograms of IPD incidence 
for the period 1979-2006 in the aggregate, and by gender, age (in ranges), and geographic area.

\section{Analysis}

IPD Incidence in the United States

The first measure obtained was the incidence levels of IPD in the United States for the period from 1979-2006, as cumulative incidence for all variable, and cumulative incidences by gender, age (in ranges), and geographic area. Using the SPSS Data Select command on the merged data set, IPD cases by ICD-9CM code, specifically pneumococcal septicemia (038.2) or bacteremia (790.7) with an accompanying pneumococcus (041.2), as well as for pneumococcal meningitis (320.1), were selected and the resulting cases counted by use of the SPSS 17.0 for Mac Analyze/Descriptive Statistics/Frequencies command (SPSS, Chicago). Calculation of the cumulative incidence for the period from 1979-2006 followed, by dividing the estimated number of IPD cases during the period from 1979-2006 by the size of the population for each of year within the period from 1979-2006 totaled. The U.S. census population information provided for each year of the NHDS data was used to establish the denominator (U.S. Department of Health and Human Services, 2008).

This process was repeated with the data stratified by gender, then repeated with the data stratified by age (in ranges) (0-4 years, 5-14 years, 15-24 years, 25-34 years, 3544 years, 45-54 years, 55-64 years, 65-74 years, 75-84, 85-98), and finally repeated with the data stratified by geographic area. The resulting information was used to establish the cumulative rate of incidence of IPD per 100,000 from 1979-2006, and the incidence rates of IPD per 100,000 by gender, age ranges, and by geographic area. 
IPD Endemic Levels in the United States

The second measure obtained was the endemic level of IPD for the period 19792006 , calculated as the summation $(\Sigma)$ of the annual incidence of IPD per 100,000 . The US census population information provided for the 28 years of the NHDS data was used as the denominator. As previously noted, the endemic level was calculated to establish epidemic levels in the presence of periodic outbreaks; periodicity was not observed, thus this level was not used in subsequent analyses.

IPD Seasonality in the United States

An analysis was intended to determine the seasonal incidence level of IPD. Using the SPSS Data Sort and Select commands on the merged data set, IPD cases by the specific ICD-9CM code for IPD were selected, and the resulting cases counted by using the SPSS 17.0 for Mac Analyze/Descriptive Statistics/Frequencies command (SPSS, Chicago). The results were then subjected to frequency domain time-series analysis through the employment of the SPSS 17.0 for Mac add-on module Forecasting. The SPSS 17.0 for Mac Forecasting command set Spectra were used to execute an FFT and plot a periodogram of IPD incidence by year in the period from 1979-2006. The data were not analyzed by the strata of age (in range), gender, or geographic area, as the analytic techniques used require long time series, and stratification into shorter groups of years would not have met this requirement. Exponential smoothing of the data was through application of the Parzen filter algorithm in the SPSS 17.0 for Mac Forecasting Spectra command set (SPSS, Chicago). 
IPD Periodicity in the United States

As in the analysis for seasonal components, the appropriate SPSS Data Sort and Select commands were applied to the merged data set, and IPD cases by the previously identified ICD-9CM codes were selected, and the resulting cases counted by use of the SPSS 17.0 for Mac Analyze/Descriptive Statistics/Frequencies command (SPSS, Chicago). The results were then subjected to frequency domain time-series analysis through the SPSS 17.0 for Mac add-on module Forecasting. The SPSS 17.0 for Mac Forecasting command set Spectra was used to execute an FFT and plot periodograms of IPD incidence for the period from 1979-2006. Periodograms were generated for the allvariables data set and for data stratified by gender, age in ranges, and geographic region. The periodograms were generated in the frequency domain, and exponential smoothing of the data was through application of the Parzen filter algorithm in the SPSS 17.0 for Mac Forecasting Spectra command set (SPSS, Chicago).

Results

IPD Incidence in the United States

The yearly incidence of IPD in the United States, for the period from 1979-2006, ranged from a low of 7.2 per 100,000, in 1985, to a high of 143.6 per 100,000, in 1998 . The annual IPD incidence rate fell from 77.3 per 100,000 in 2000 to 26.1 per $1,000,000$ in 2004 , then increased over the final two years of the data examined to 49.7 per 100,000 (see Figure 1). The cumulative incidence of IPD in the United States for the period from 1979-2006 was calculated as 53.5 per 100,000 . 


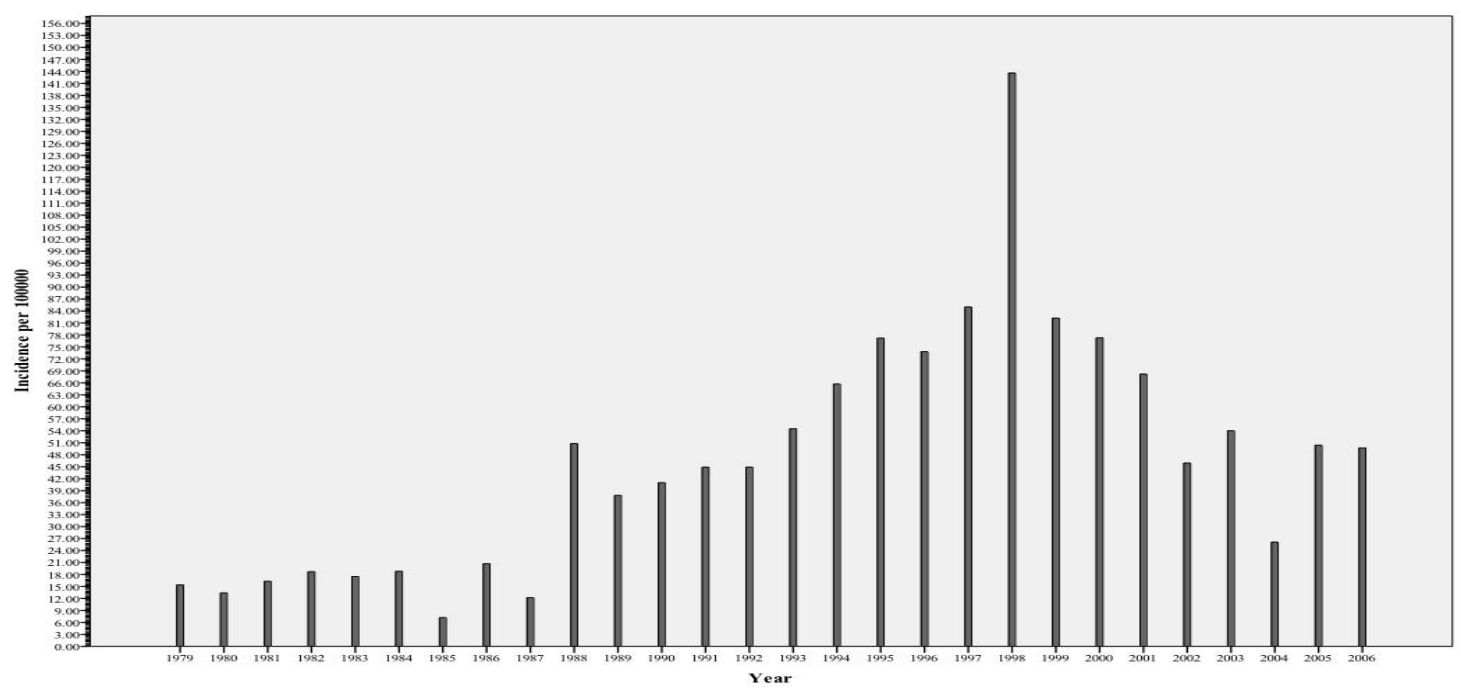

Figure 1. Incidence of IPD in the United States, 1979-2006, per 100000.

Stratification by Age Group, Gender, and Region

Stratification by age (in ranges) revealed that persons aged from 5-14 years had the highest IPD incidence rate, 133.2 per 100,000, while those persons aged from 15-24 had the lowest incidence rate, 15.9 per 100,000 (see Figure 2). When stratified by gender, the cumulative incidence of IPD in the United States from 1979-2006 was 68.0 per 100,000 for men and 43.7 per 100,000 for women (see Figure 3). An examination of data by geographic region revealed that the Midwest region had the highest incidence in the period from 1979-2006 with an incidence of 79.0 per 100,000. The Northeast and South regions both had an incidence of 66 per 100,000 in the same period. Finally, the West region had the lowest IPD incidence of 58.9 per 100,000 (see Figure 4). 


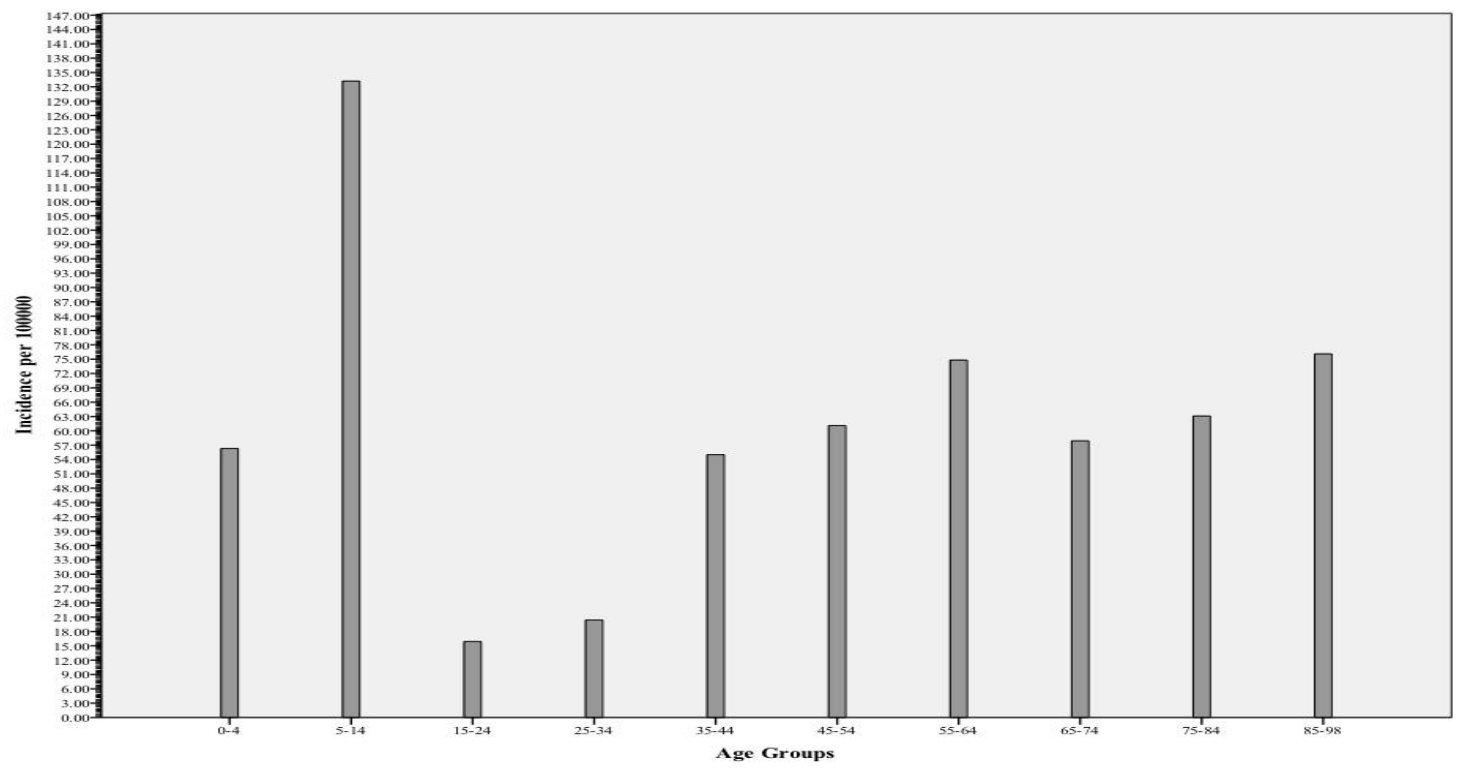

Figure 2. Incidence of IPD in the United States, 1979-2006, by age.

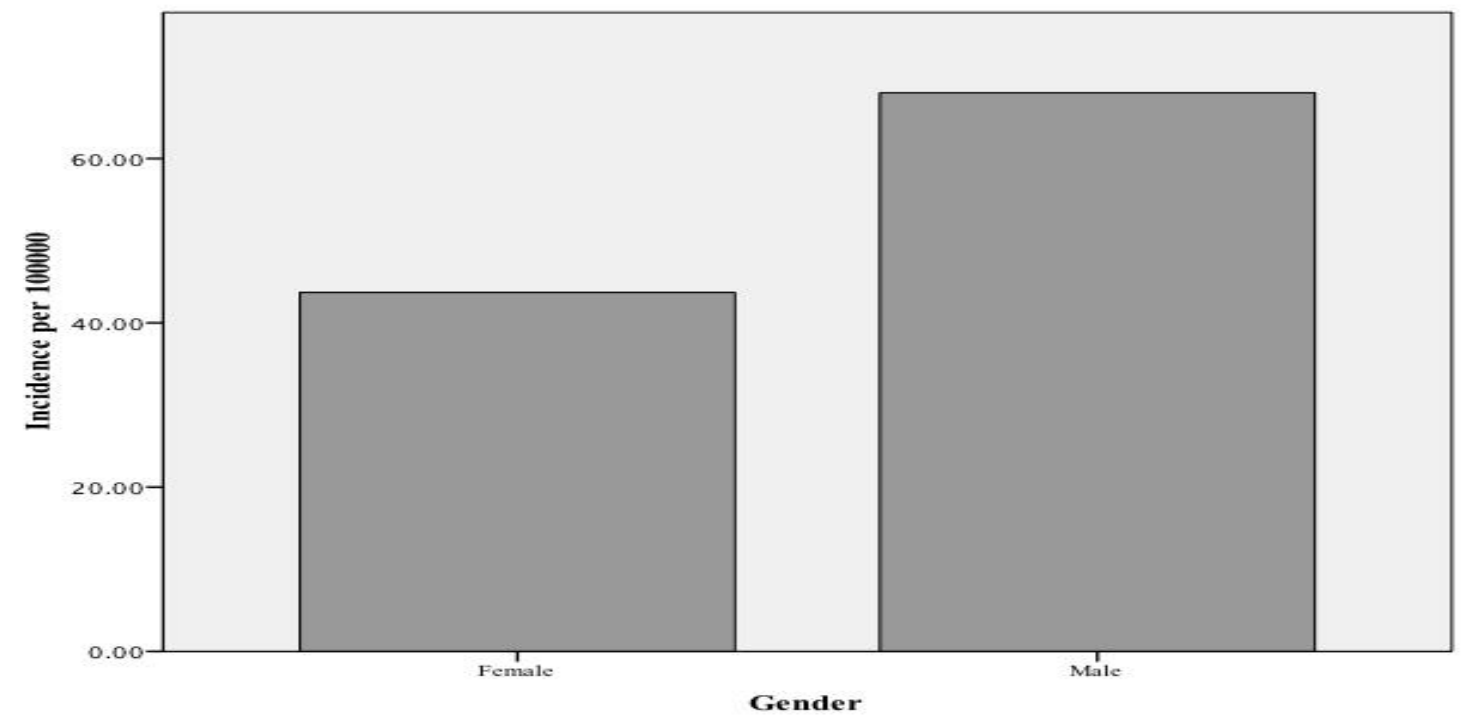

Figure 3. Incidence of IPD in the United States, 1979-2006, by gender. 


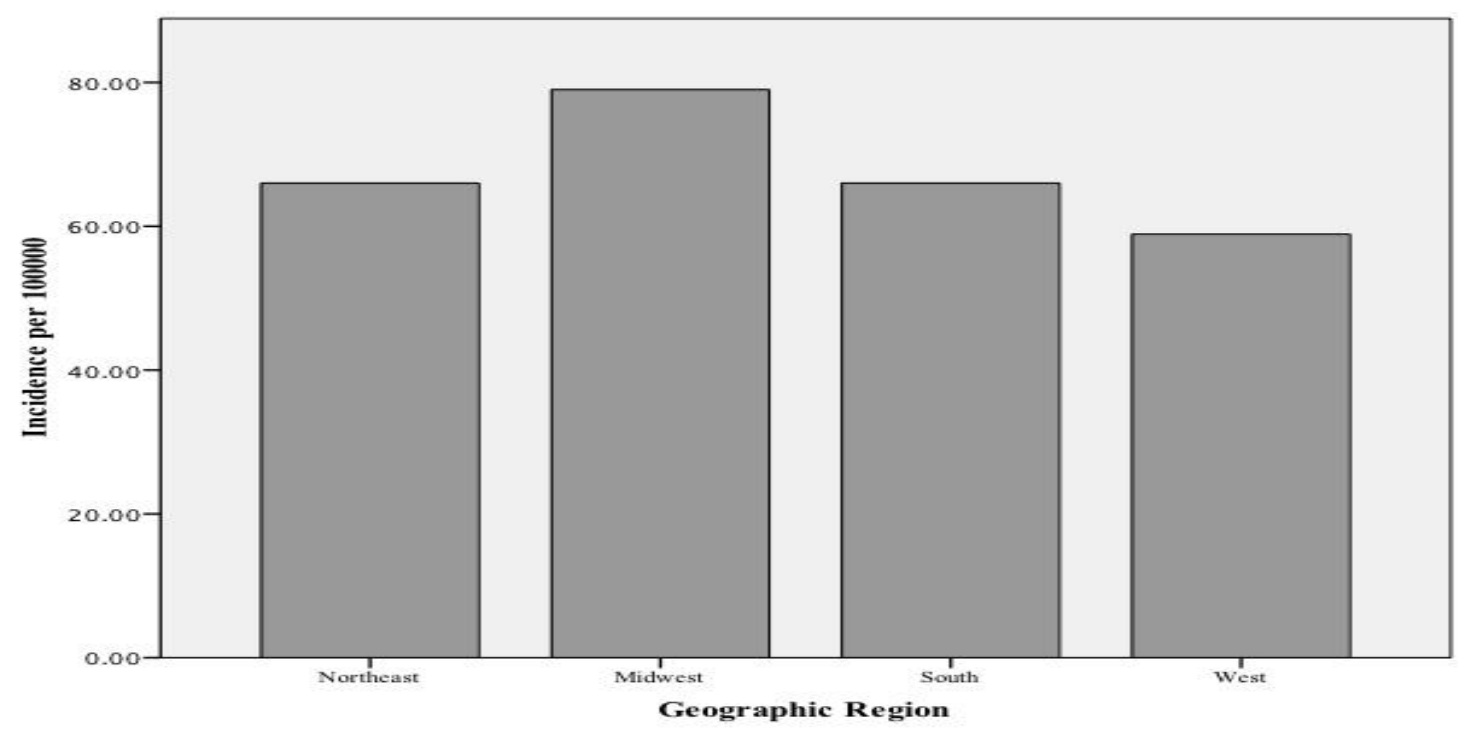

Figure 4. Incidence of IPD in the United States, 1979-2006, by geographic region. IPD Endemic Levels in the United States

The endemic level of IPD in the United States for the period 1979-2006 was 46.9 per 100,000. The endemic level was calculated to enable establishment of epidemic levels in the presence of periodic outbreaks. Because the analysis did not detect periodic components to the epidemic dynamic, this level was not used in subsequent analyses. Periodicity of IPD Epidemics in the United States.

The periodogram for IPD incidence in the United States for the period from 19792006 by month, combining all ages, genders, and geographic regions is shown in Figure 5. This addresses hypotheses $\mathrm{H}_{\mathrm{ol}}$ and $\mathrm{H}_{\mathrm{a} 1}$. This periodogram exhibits a disordered waveform not consistent with periodicity, supporting the null hypothesis. This result held even after exponential smoothing of the sample spectrum. None of the perturbations in the waveform were of sufficient amplitude (represented by vertical height of the centered wave along the y-axis) to warrant further examination. 


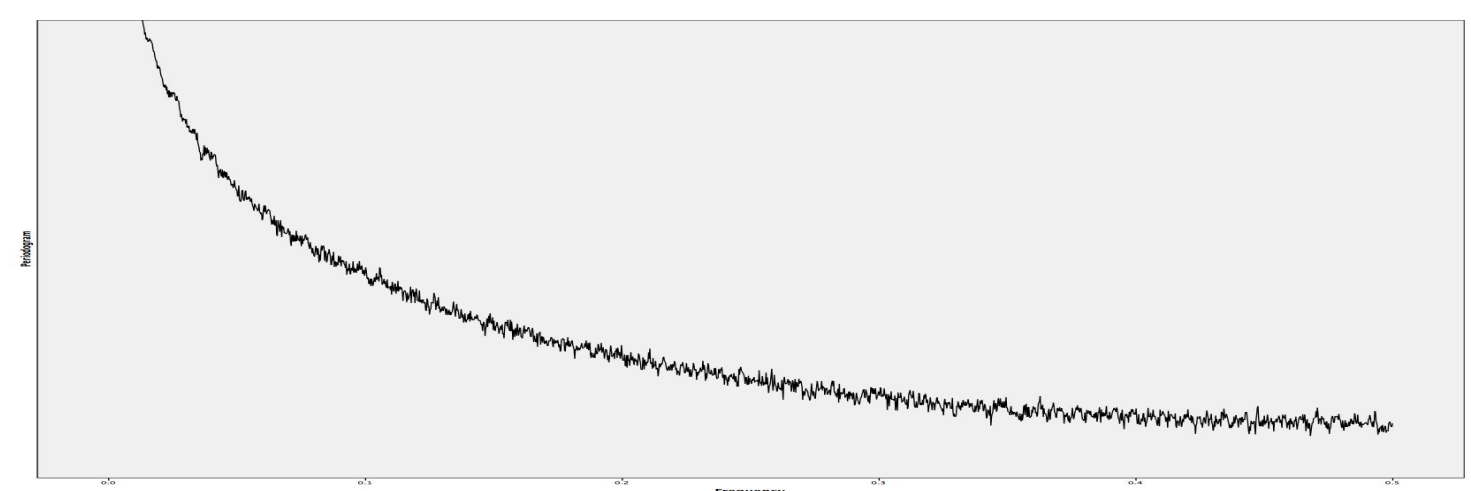

Figure 5. Periodogram of IPD incidence in the United States for the period from 19792006, inclusive of all ages, genders, and geographic regions.

IPD periodicity stratified by gender, geographic region, and age group

Periodograms for IPD cases stratified by gender are shown in Figures 6 and 7 .

These graphs focus on hypotheses $\mathrm{H}_{\mathrm{a} 2}$ and $\mathrm{H}_{\mathrm{o} 2}$, and yielded similar results as the overall incidence periodogram; that is, the waveforms are not consistent with periodicity of IPD epidemics, even after exponential smoothing of the sample spectrum. No peaks consistent with periodic events could be identified, and no perturbations in the waveform were of sufficient amplitude (represented by vertical height along the y-axis) to warrant further examination. Despite the gender disparity in incidence, the morphology of these periodograms were similar in that both showed a similar lack of peaks consistent with periodic components that could be analyzed further. 


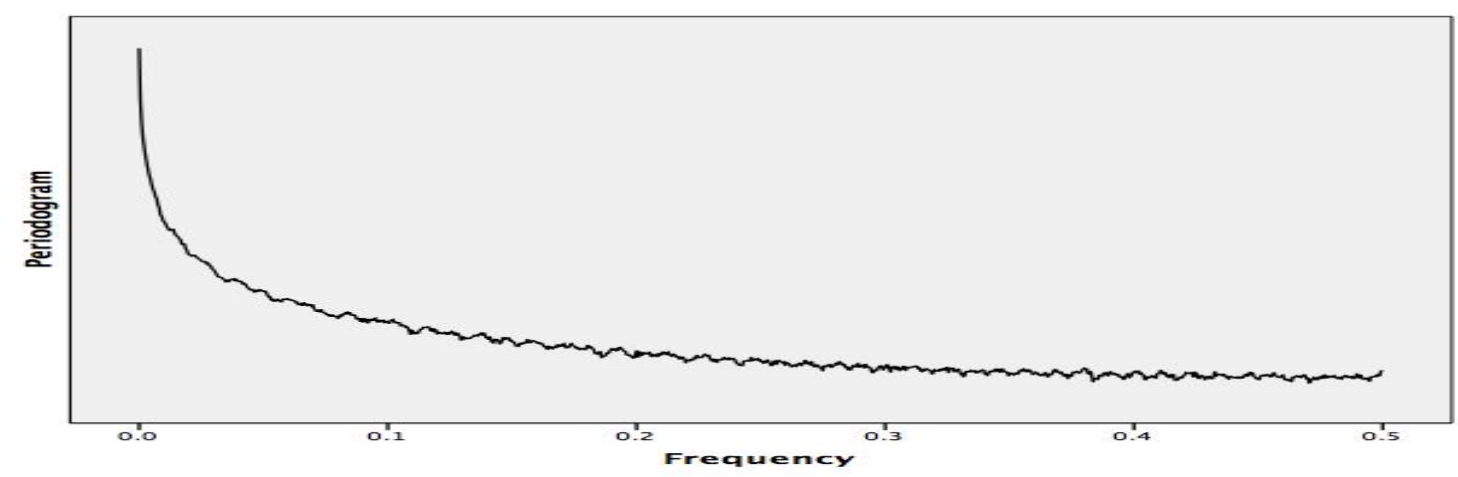

Figure 6. Periodogram of IPD incidence in the United States for the period from 19792006, by gender, male.

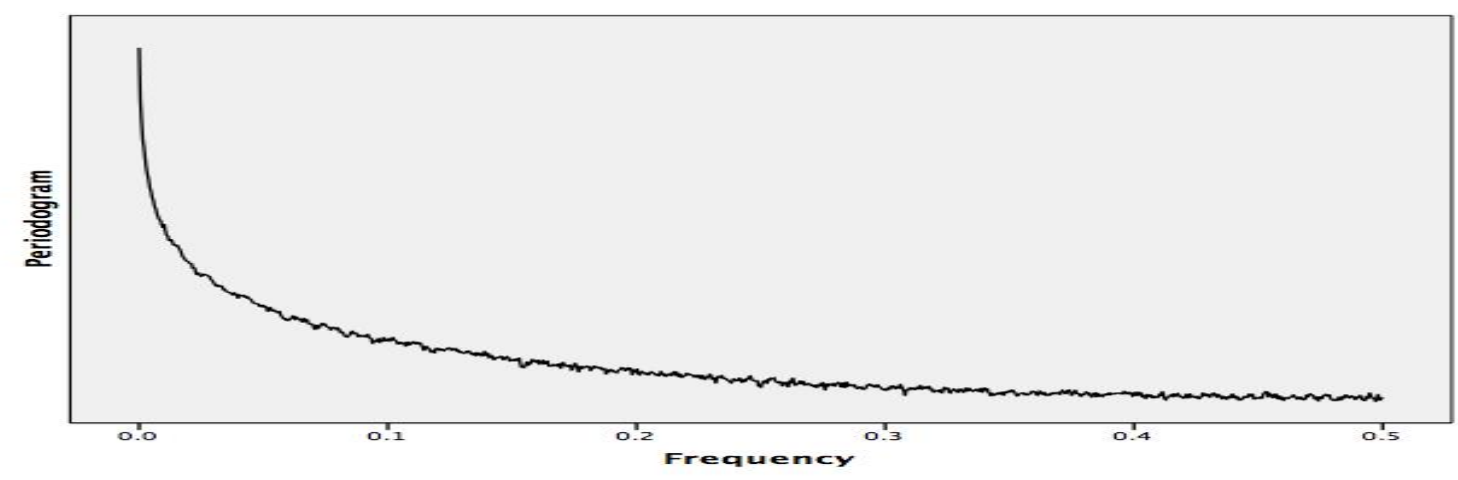

Figure 7. Periodogram of IPD incidence in the United States for the period from 19792006, by gender, female.

The time series analysis graph for periodicity of IPD epidemics in the United States, 1979-2006 by geographic regions (see Figure 8), for hypotheses $\mathrm{H}_{\mathrm{o} 3}$ and $\mathrm{H}_{\mathrm{a} 3}$, did not reveal a recurrent epidemic pattern in any of the four regions. The morphology of the waveforms in these periodograms differs from the previous examples by exhibiting a reduced density of waveforms. None of the periodograms contain a peak or perturbation consistent with a recurrent epidemic pattern 


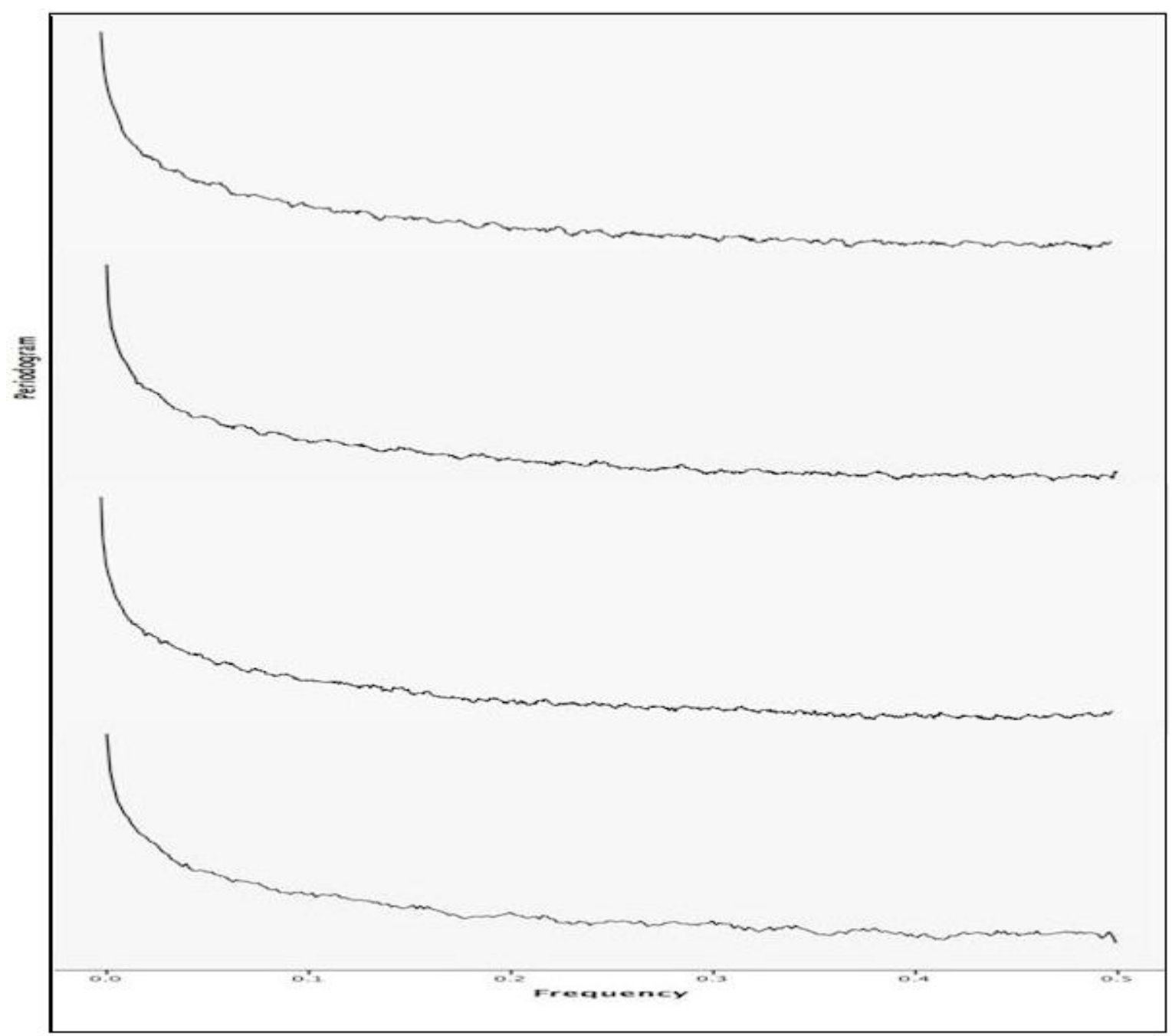

Figure 8. Periodogram of IPD incidence in the United States for the period from 19792006, Northeast, Midwest, South, and West census regions (from top to bottom).

The results of the analysis of the occurrence of IPD cases stratified by selected age ranges, attending to hypotheses $\mathrm{H}_{04}$ and $\mathrm{H}_{\mathrm{a}}$, are shown in figure 9 , as well as in figures $11,12,13,14$, and 15, which may be found in the appendix. Again, the waveforms 
for each age group are not consistent with periodicity of IPD epidemics, even after exponential smoothing of the sample spectrum. As in the analysis by geographic region, the morphology of the waveforms in several of the periodograms differs from those in the aggregate by displaying a reduced density of waveforms. This is true for the results of time series analysis of IPD incidence for ages ranging from 5-14 years (see Figure 11), 15-24 years (see Figure 12), 25-34 years (see Figure13), 35-44 years (see Figure 14) and 45-54 years (see Figure 15). Periodograms 11-15 may be found in the appendix. The waveforms for theses age ranges were not conducive to analysis, and the low amplitude of these waveforms most likely reflects the distribution of IPD by age group. In all age ranges that displayed periodograms suitable for analysis, no peaks consistent with periodic events could be identified, and no perturbations in the waveform were of sufficient amplitude (represented by vertical height along the y-axis) to warrant further examination. 


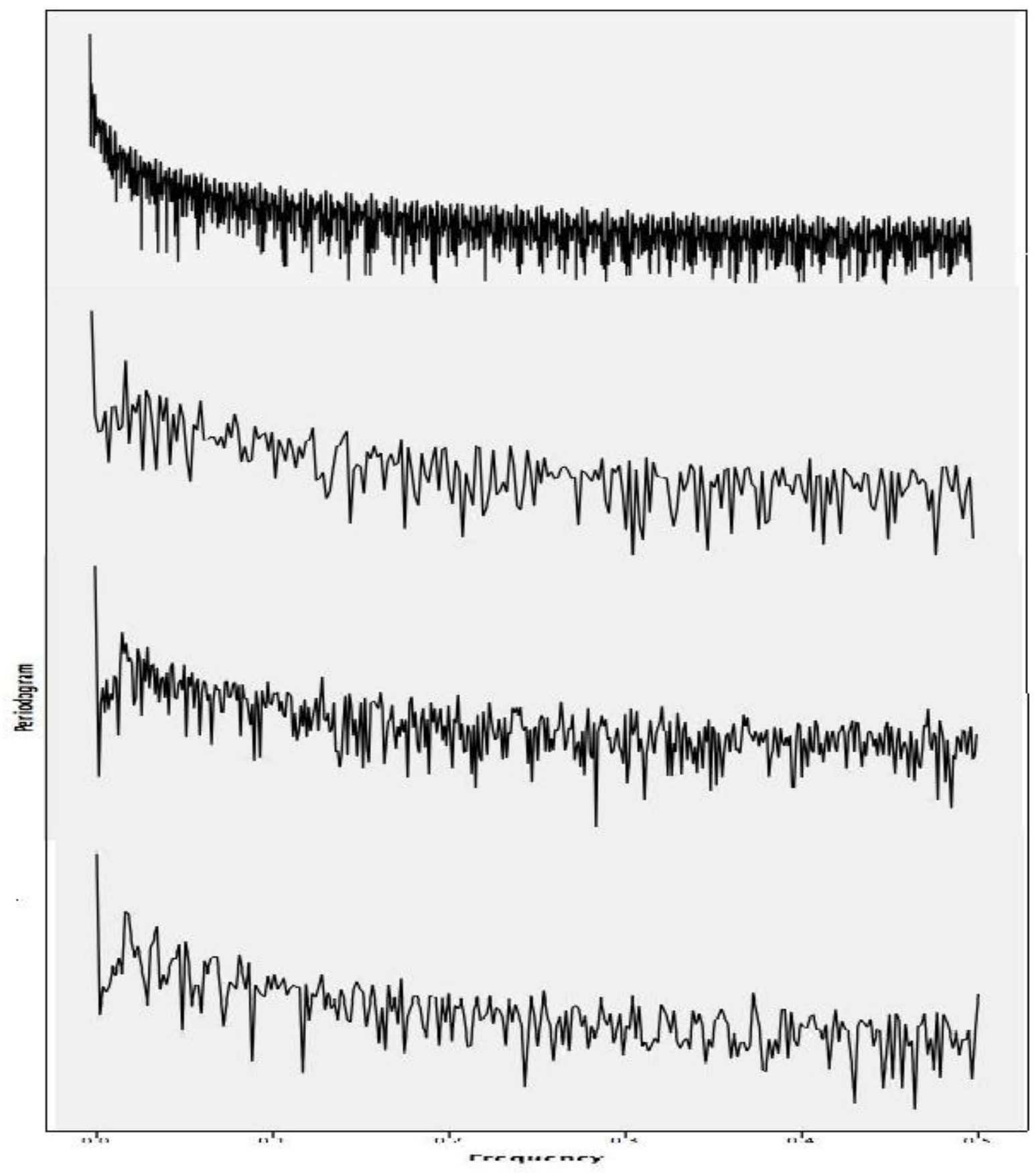

Figure 9. Periodogram of IPD incidence in the United States for the period from 19792006, 0-4 years of age, 55-64 years of age, 65-74 years of age, 75 years of age and older (from top). 
When analyzed for seasonality, the same results held; that is, no discernable peak was found in the periodogram consistent with recurrent events (see Figure 10).

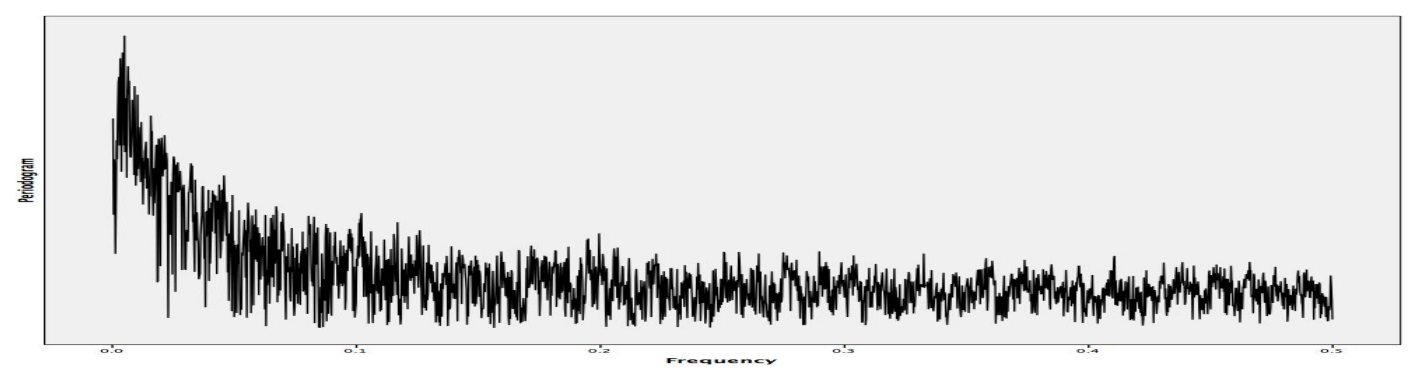

Figure 10 . Periodogram of IPD incidence in the United States for the period from 19792006, analyzed for seasonality of outbreaks.

\section{Summary}

The results of this study fail to support the hypotheses that IPD occurred in periodic epidemics of a definable frequency in addition to the predicted seasonal increase in incidence in the United States during the period from 1979-2006, in the aggregate, and when stratified by age, gender, or geographic region. Additionally, the results of this study do not support a seasonal component to IPD outbreaks. While some variance in incidence rates from previous studies was observed, the cumulative rates of incidence for IPD in the United States were consistent with previously reported data. The age-related distribution of IPD noted by prior researchers was, however, also discernable in the data analyzed in this study. Further discussion of the results of this study and its implication for prediction of IPD epidemics will be presented in chapter 5. Also in the following chapter is a review of potential lines of inquiry generated by this study, and a discussion of the positive social impact that results from this study. 


\section{CHAPTER FIVE: DISCUSSION}

\section{Overview}

Streptococcus pneumoniae, in its manifestation as IPD, has been and still is responsible for significant morbidity and mortality (Bloom, Wheeler, \& Lynn, 1999; Centers for Disease Control and Prevention, 2006; Dochez and Avery, 1915; McDaniel and Swialto, 2004; Osler, 1898). Our understanding of IPD epidemic dynamics currently extends only as far as annual seasonality (Dowell, Whitney, Wright, Rose, \& Schuchatt, 2003), which limits our ability to plan, execute, and evaluate preventive measures. Additionally, changes in the bacterial genome have conferred new antibiotic resistance patterns (Lopex, 2006), and permitted the emergence of serotypes that are not addressed by the current vaccines. Our limited ability to predict IPD epidemic dynamics creates challenges in detecting these changes. It was the unclear nature of epidemic patterns in IPD outside of the previously described annual outbreaks, and the impact of the absence of understanding of IPD epidemic dynamics that led to this research study.

In order to elucidate the epidemic pattern, a retrospective cohort study was performed, resulting in a 28-year retrospective description of IPD epidemic dynamics. The study set out to establish the existence or absence of a recurrent epidemic pattern and frequency, with the intent of enhancing our understanding of IPD. The following research questions were addressed:

Do epidemics of IPD occur in a periodic manner with a definable frequency in addition to the predicted seasonal increase in incidence,

(1) in the aggregate,

(2) by gender, 
(3), by age range , and

(4) by geographic area.

Two specific objectives were addressed in order to address the following questions: What was the incidence level of IPD for each year and for the combined period in the time span from 1979-2006? and What was the endemic level of IPD in the United States for the period from 1979-2006 in the aggregate, and by gender, age (in ranges), and geographic area?

The results of the study were clear; the null hypotheses held in all cases and in all strata. IPD in the United States, for the period from 1979-2006, did not occur in periodic epidemics of a definable frequency outside of the predicted seasonal increase in incidence. Additionally, the seasonality described in a previous study was not detected by our analysis.

\section{Discussion}

\section{Incidence and Endemicity}

As a basis for examining the periodicity of IPD epidemics, incidence and endemicity rates were determined. Calculation of incidence levels permitted the comparison of the results of this study to those of previous studies. Additionally, this calculation provided a means to detect the inadvertent inclusion of cases that were not clearly pneumococcal in nature. In the study by Danai et al. (2007), inclusion of nonpneumococcal causes of sepsis resulted in a rise in incidence over time. Should this have occurred in this study, the rise in incidence would have been contrary to the expected fall in incidence, particularly in the period after introduction of the pediatric vaccine (Hammitt et al., 2006; McBean, Park, Caldwell, \& Yu, 2005; Moore et al., 2004; 
Poehling et al., 2006; Shah \& Ratner, 2005; Tsai, Griffin, Nuorti, \& Grijalva, 2008). The endemic level was calculated to enable establishment of epidemic levels in the presence of periodic outbreaks; periodicity was not observed, thus this level was not used in subsequent analyses.

The results of the incidence calculations were consistent with previous studies, with the exception of the age-stratified analysis. The results are also congruent with previously reported studies which documented incidence rates ranging from 8-75 cases per 100,000 (Brueggemann, Peto, Crook, Butler, \& Kristinsson, 2004). Significantly, the annual incidence rates found in this study show a decline in the IPD incidence rate which is consistent with previously reported declines, after implementation of the pediatric vaccine (Hammitt et al., 2006; McBean, Park, Caldwell, \& Yu, 2005; Moore et al., 2004; Poehling et al., 2006; Shah \& Ratner, 2005; Tsai, Griffin, Nuorti, \& Grijalva, 2008). Thus, a major purpose of incidence calculation was met, and the results of this study can be compared to a previous study addressing epidemic dynamics of bacteremia.

The pattern of IPD incidence rates in the aggregate observed over the time period after implementation of the pediatric vaccine is consistent with a sample that included only streptococcal disease. The results of this study were likely unbiased by the inclusion of other pathogenic causes for sepsis, meningitis, or bacteremia. Thus, the overall incidence and endemicity rates observed in this study establish a robust basis for the subsequent time series analysis. However, before discussing the findings of the time series analysis, further attention should be given to the incidence findings.

Examination of the data by the strata of gender, geographic region, and age (in ranges) revealed variations in incidence rates, largely consistent with those reported by 
previous studies (Christenson, Lundbergh, Hedlund, \& Ortqvist, 2001; Harboe, Thomson, Riis, Valentiner-Branth, Christensen, et al., 2009; Huang, Finkelstein, \& Lipstich, 2005; Kyaw et al., 2005; McDaniel \& Swialto, 2004; Robinson, 2001). When examining the results of analysis of specific geographic region data, the regional variations in IPD incidence supported the observations of Huang, Finkelstein, and Lipstich (2005), who reported geographic variation in prevalence of pneumococcal carriage (a precursor to pneumococcal disease). Also supporting the findings in previous work is the detected disparity of incidence between genders, with males displaying a higher incidence of IPD than females (Harboe, Thomson, Riis, Valentiner-Branth, Christensen, et al., 2009). The reasons for this disparity are unclear, though this finding is concordant with those of two other studies. A study of immunocompetent adults (Nuorti et al., 2000) found that male gender was an independent risk factor for IPD. The incidence of IPD was higher in male infants and children in a study conducted by Klein (1981). However, an examination of natural immunity to the pneumococcus in light of age and gender revealed that, with aging, a stronger decline in anti-pneumococcal antibody concentration was seen in women when compared to men (Simell, Lahdenkari, Reunanen, Kaeyhty, \& Vaevaeinen, 2008).

The higher IPD incidence in the 5-14 year age group differs from study results reported by Robinson (2001), in which the highest incidence rates were in children under the age of two. However, the results for adults aged 80 years and greater, ranging from 63.1 to 76.1 per 100,000 are congruent with those reported by Kyaw et al. (2005) of 71.2/100,000 (Kyaw et al., 2005). Despite differences in reported incidence in the lower age ranges between this and previous studies (Christenson, Lundbergh, Hedlund, \& 
Ortqvist, 2001; McDaniel \& Swialto, 2004), the previously described bimodal distribution of IPD incidence (disease affecting primarily those early and late in life) was apparent in the results of the analysis in this study (Figure 2). The difference is attributable to age ranges between the previous studies, and this one may reflect differences in contact patterns between the sample population in the previous studies and the sample population in this study (Bansal, Grenfell, \& Meyers, 2007). That is, because the studies sampled from different populations and over different time spans, there might have been geographic and temporal difference in the number and intensity of contacts and exposures in different social settings, which would have influenced the spread of IPD.

Streptococcus pneumoniae is an adaptive organism, able to shift genetically in response to host pressures and to share resistance genes with and from other organisms (Boots et al., 2003; Lopex, 2006; Sorrell, Pedersen, Hails, \& Boots, 2009), providing plausible mechanisms for the continued universal colonization of $S$. pneumoniae and the continued endemicity of IPD. Further ensuring the persistence of colonization and disease are the nature of the bacterium and of the vulnerable population. Not all colonization with S. pneumoniae is followed by infection, not all infection with $S$. pneumoniae is fatal, and infection with one strain of S. pneumoniae does not confer resistance to other strains of the organism. This characteristic of the pneumococcus permits the persistence of the bacterium in the host population. This persistence of non-pathenogenic colonization allows the bacterium to reproduce more successfully than a more virulent pathogen might, but also ensures that disease endemicity is preserved (Boots et al., 2003; Sorrell, Pedersen, Hails, \& Boots, 2009). 
Host contact patterns also bear on endemicity. As observed in this and previous studies (Huang, Finkelstein, \& Lipstich, 2005), the incidence of IPD varies across the regions of the US. This variation in rate may also be due to differences in contact patterns (Bansal, Grenfell, \& Meyers, 2007); these differences in the number, frequency and intensity of contacts and exposures in different social settings prevent complete eradication of $S$. pneumoniae colonization and thus preclude eradication of IPD. What Hagenaars, Donnelly and Ferguson (2004) call between patch transmission is unpredictable, and could serve to prevent IPD from extinction.

These factors partially explain the rates of incidence and continuing endemicity observed in this study. Another factor impacting the results of this study is the possible presence of anomalies in reporting to the NHDS and resulting variation in the data sets used for analysis. The initial time periods subjected to analysis, 1979-1987, and most noticeably, the years 1985 and 1987, were marked by lower incidence rates than reported in previous studies. It is possible that in the first few years that this finding reflects differences in the use of ICD-9CM codes during those periods, which followed a significant change in the coding guidelines; that is, the change from the ICD-8 in 1979 (Popovic, 2001). However, within the NHDS documentation, no reference to changes in ICD-9CM codes or coding practices were found that impacted on the years 1985 and 1987, and this is not a limitation of the study.

A more likely explanation for those years is that of an under-reporting or misclassification of IPD during those time periods. Under reporting in the NHDS has been documented for other disease classifications (Surjan, 1999; Movig, Leufkens, Lenderink \& Egberts, 2003; van de Garde, Oosterheert, Bonten, Kaplan \& Leufkens, 
2007). However, within the NHDS documentation, insufficient information on reporting practices or changes in the time span of interest prevents more than speculation.

Later incidence rates are more consistent with those reported in earlier studies (Bridy-Pappas et al., 2005; Brueggemann, Peto, Crook, Butler, \& Kristinsson, 2004; Hammitt et al., 2006; McBean, Park, Caldwell, \& Yu, 2005; Moore et al., 2004; Poehling et al., 2006; Shah \& Ratner, 2005; Tsai, Griffin, Nuorti, \& Grijalva, 2008). Whether this reflects changes in coding or reporting practices subsequent to 1987 could not be determined. It must be noted, however, that under-reporting, while it might have impacted the incidence rates in the first eight years of the study period, should not have impacted the frequency-domain time series analysis that examined both seasonality and periodicity (Keeling \& Rohani, 2008b). A resistance to the effects of under-reporting influenced the decision to employ this analytic technique.

Additionally, the mean incidence rate and the resulting endemic rate calculated from the data used in this study are congruent with those reported by other researchers. This overall consistency contributes to confidence in the data set as a basis for time series analysis. Thus, the data sets were used as a basis for time series analysis in the frequency domain. These analyses were conducted in an attempt to discern both seasonal and additional periodic epidemic dynamics.

\section{Seasonality}

Numerous studies have shown that IPD has a seasonal pattern of incidence; this pattern directly correlates to seasonal outbreaks of viral respiratory disease, and with variations in the length of the day (Ampofo et al., 2008; Butler \& Schuchat, 1999; Kim et al., 1996; Stegemann et al., 2009; Talbot et al., 2005). These influences on IPD 
seasonality leads to a peak incidence in the winter months (Dowell, Whitney, Wright, Rose, \& Schuccat, 2003; Kim et al.; Talbot et al.). However, the results of this study revealed findings inconsistent with this previous work, in that IPD in the United States for the period 1979-2006 for all variables did not occur on a predictable seasonal basis.

The explanations for the lack of seasonality of IPD outbreaks in this study are many. Prior infection with viral agents, changes in host contact patterns, implementation of vaccination programs, and other changes in environmental factors have all been put forward as possible causes for alterations in the seasonality of diseases (Fisman, 2007), and IPD is likely to have been influenced by many or all of these factors over the 28 -year time period subjected to analysis. Additionally, the length of time addressed by this study might have influenced the results.

In a previous study that used time series analysis to detect seasonality in IPD, Dowell et al. (2003) performed time series analysis for two years of data, in contrast to this study, which evaluated outbreak dynamics over a 28 -year period. It is possible that alterations in the host/pathogen ecology over the longer period of time precluded or obscured the emergence of a seasonal pattern that was persistent over the entire analysis period (Watts, Muhamad, Medina \& Dodds, 2005). The time span addressed by this analysis, 28 years, should have been sufficient to reconstruct the dynamics of the system and identify any seasonal components or other periodicities (Kamlapriya, Thiel, Romano, Marwan, Schwarz \& Kurths). The observed absence of a predictable, recurrent pattern of IPD epidemic dynamics was also the finding when the data was analyzed for dynamics beyond an annual occurrence. 
Periodicity

Results from the time series analysis revealed findings that fail to support the existence of periodic epidemics of IPD in addition to the expected annual increases in incidence. These results hold for the analysis in the aggregate, and for data stratified by gender, age (in ranges), and by geographic region. Examination of the incidence data (Figure 1) with attention to the shape of the data points over time supports this conclusion, as the variation in levels displays no discernable pattern. Use of frequency domain time series analysis techniques should have revealed any hidden patterns or periodic components (Wei, 1994f). Instead, the results of this study showed an absence of IPD periodic epidemics of a definable frequency outside of the predicted seasonal increase in incidence in the United States for the period from 1979-2006, in the aggregate, and when stratified by gender, geographic region, or age (in ranges). When examining the periodograms for the strata, differences in morphology are apparent when compared to the aggregate periodogram, most likely due to reduced case counts in the stratified analyses. However, with the exception of the 5-54 years age strata, insufficient waveform density was present to permit conclusions. None of the analyzed periodograms whether in the aggregate or by strata, displayed recurrent events consistent with periodic epidemics. Therefore, the results of this study reveal that IPD epidemics demonstrate a chaotic dynamics; that is, a discrete non-Markov process (Yulemetyev et al., 2003). IPD appears to exhibit aperiodic epidemic behavior.

Frequency domain analysis produced visual representations of the quantitative results of the Fourier Transforms which facilitated the analysis of the frequency domain time-series (Wei, 1994a) that correlated to the contribution of the varied frequency 
components to the observed patterns of disease incidence (Anderson et al., 1984; Lindsey, 2004a). Theses periodograms reveals waveforms that were chaotic, that is, in a state of disorder and irregularity (Yulemetyev, Yulmetyeva, \& Gafarov, 2005). The periodogram of aggregate data displays what Yulemetyev, Emalyanova, Demin, Gafarov, Haenggi and Yulmetyeva (2003) described as "strongly expressed asymmetry," and evidence for the "existence of a clearly expressed noise."

This finding is consistent with the findings of Danai et al. (2007), in a study of sepsis of all causes. Much like the factors addressed in the discussion of the seasonality findings, variations in host contact patterns, lower incidence rates following the release of the pediatric vaccine, changing antibiotic resistance patterns, and the emergence of NVT serotypes could have acted to attenuate the pattern of, or completely prevent the emergence of, a pattern of IPD epidemics over longer periods (Watts, Muhamad, Medina \& Dodds, 2005). If the pattern was only weakly established, and lacked persistence, the effects of changes in host immunity and the efforts of the microbe to remain endemic might either eliminate or prevent patterns from forming (Bansal, Grenfell \& Meyers, 2007). Determining and estimating these effects can be difficult (Lande, Engen \& Saether, 2002), and the analytic technique chosen for this study was not suited to that task (Rodriquez-Aria \& Rodo, 2004).

In a random system, where the disease is endemic, the influences of stochastic events (such as a new pool of susceptible subjects) on the normal random state can adjust the chaotic and random behavior towards regularity (Yulemetyev, Yulmetyeva, \& Gafarov, 2005). This did not appear to happen in IPD epidemic dynamics in the data set analyzed in this study. However, modeling of the influence of stochastic events on 
epidemic dynamics can be difficult, because these chance events might be major factors in the eventual size and timing of an epidemic (Finkenstadt, Bjornstad \& Grenfell, 2002; Mollison et al., 1994).

In addition to these seasonal variations in contact patterns, replenishment of susceptible hosts, colonization with S. pneumoniae, and occurrence of IPD can be reasonably expected to occur stochastically, and influence the dynamics of the system (Xia, Bjornstad, \& Grenfell, 2004) by introducing a degree of uncertainty (Koelle \& Pascual, 2004). An example of a stochastic event that might have acted as a weak attractor in IPD dynamics is the irregularity in pediatric vaccine supply and variations in vaccine effectiveness, such as from low efficacy vaccines or serotype replacement (Alexander, Moghadas, Rohani, \& Summers, 2006). These factors could have affected the epidemic dynamics of IPD by providing an impact to the system that was continued through the remainder of the time period; however, it does not appear that this occurred.

Any impact of these possible stochastic events on the time series of IPD incidence did not exhibit persistence; that is, the impact of increased IPD incidence in 1998 or of a later shortage in the pediatric vaccine did not exert an ongoing effect on later incidence levels in the series (McCabe, Martin \& Tremane, 2005). This might have resulted from temporal differences in exposure patterns and interactions between susceptible and infectious individuals (Watts, Muhamad, Medina \& Dodds, 2005; Wallinga, Edmunds \& Kretzschmar, 1999); the changing disease ecology could have prevented the emergence of stable epidemic cycles as a result of stochastic events (Rodriquez-Arias \& Rodo, 2004). 
The impact of stochastic changes in the overall ecology of a disease on epidemic dynamics can be seen in measles, in which seasonality was less striking and epidemic periods changed after the introduction of vaccination (Grenfell, Bjoernstadt \& Kappey, 2001). It can be speculated that the geographic areas addressed in this study of IPD were larger than those in the Grenfell, Bjoernstadt and Kappey study, and the variations in contact pattern less predictable, thus the impact on any seasonality or periodicity might be larger, as well. Furthermore, as vaccination levels increase, periodic cycles of disease can decrease (Anderson, Grenfell \& May, 1984). All of these factors could partially explain the absence of seasonality or periodicity in IPD in this study.

Another factor, the previously discussed possible variation in reporting of IPD, while it might have impacted the incidence rates reported in this study, should not have influenced the results of the time series analysis. When planning this study, consideration was given to the regularity or irregularity of observations of a key variable, the count of cases of IPD, and the analytic technique of frequency-domain time series analysis was chosen in part to account for this possibility. Under-reporting and the resulting lower amplitude of any periodic events should not have prevented detection patterns to these events by frequency domain analysis (Wei, 1994b). Any periodicity and frequency of IPD outbreaks should have remained detectable even in the face of under-reporting of events (Keeling \& Rohani, 2008b); the same possible under-reporting that might account for the appearance of reduced incidence in the early years of the data.

The appearance of reduced incidence could have lead to the phenomenon of disease fadeout (the extinction of a disease expressed in a mathematical model, most likely as a result of a sufficient drop in the number of infected and susceptible individuals 
in a given population within the model), which has been a concern when constructing models and analyzing data in these situations, ( Keeling \& Rohani, 2008b; Lloyd, 2004). This did not appear to occur in this study, perhaps due to the ubiquity of $S$. pneumoniae colonization, the emergence of NVT serotypes, and the other factors that mediate in favor of IPD endemicity.

However, all of these factors could have created observational noise that affected the results of the analysis. Observational noise (Chen \& Bokka, 2005; Keeling \& Rohani, 2008b) exhibited in an epidemic system can be difficult to separate from chaos (Stewart, 2002), and this drove selection of the analytic technique. Rohani et al. (1999) observed that pertussis is very sensitive to stochasticity, and it is possible that IPD also exhibits this sensitivity, thus obscuring or precluding the emergence of a predictable epidemic pattern. The infectious period of $S$. pneumoniae could have generated multiple small amplitude variations, with none of the variations of sufficient amplitude or persistence to develop into a cyclical pattern (Altizer, Dobson, Hosseini, Hudson, Pascual \& Rohani, 2006).

Additionally, stationarity of the epidemic dynamic and the possibility of a Markov process were considered. Epidemics exhibit non-stationarity (Keeling \& Rohani, 2008b), and no evidence could be found in the literature that IPD epidemics follow a Markov process. Examination of the periodograms revealed findings consistent with this premise, as stationarity did not appear, and no evidence of Markov processes could be found.

In an existing model of IPD dynamics, Sutton, Banks \& Castillo-Chavez (2008) have applied the Susceptible-Exposed-Infected-Recovered (SEIR) model to IPD, with the inclusion of seasonality to result in a Temporally Forced Model. In this model, colonization rates serve as a proxy for exposure, and birth rates, death rates, and 
vaccination rates are factored into the model, as is seasonality (Sutton, Banks, \& CastilloChavez, 2008). The data used to calculate rates and modify model parameters can be obtained from existing literature or direct measurement. The results of this study can be applied to this model, by exception rather than by addition to the existing model.

Specifically, the model's transfer rate equation does not require modification based upon the results of this study. Predicted birth rates, death rates, and a transfer rate that accounts for seasonality remain the most suitable predictive components of the model. This SEIR model of IPD dynamics can be linked together as sequential realizations, with the rate of entry into the population adjusted between each annual realization to account for varying birthrates, either historical or predicted. The existing model already provides the opportunity to input the vaccination rate (Sutton, Banks, \& Castillo-Chavez, 2008), which again could be modified for each realization. Realizing the entire model as a sequential linkage of individual models ameliorates the concern over use of discrete time data in a continuous dynamical system. Each annual model would retain its continuous dynamic. However, based on the results of this study, no adjustment need be made for fluctuations or perturbations in the epidemic dynamic beyond seasonality.

\section{Implications for Social Change}

The results of this study have several implications for social change. Should an epidemic pattern have been identified, it could have been used to modify an existing model of IPD epidemic dynamics to permit prediction of outbreaks beyond annual seasonality. However, in the absence of a discernable pattern, additional support is provided for maintaining and perhaps expanding the current method of surveillance, the 
Active Bacterial Core Surveillance System, which emphasizes reporting of serotype and strain (CDC, 2009), rather than prediction of future epidemic dynamics. Furthermore, the use of long-run probability calculation (Kachigan, 1982) for IPD epidemic forecasting is not supported by the findings of this study, and efforts and funding can be devoted to other, proven methods of IPD reduction. This promotes positive social change through emphasis on current, proven methods of evidence-based practice, resulting in improved health outcomes and decreased health care costs (Ament et al., 2000).

The current emphasis on mass vaccination by risk group, as opposed to vaccination for a predicted outbreak, is sound practice in light of the results of this study. Currently, prevention interventions targeted against IPD emphasize vaccination, particularly in the pediatric population (Overturf, 2000; Parsons \& Dockrell, 2002). Although not universal, vaccination has been successful in lowering the rate of IPD incidence; this reduced incidence was found during data analysis in this study. Because a pattern of IPD epidemics was not found in this study, vaccination campaigns can provide accurate information on the unpredictability of IPD to unvaccinated subpopulations. Those unvaccinated individuals relying on game-theoretical approaches to disease prevention, whether conciously or unconciously (Bauch, 2005) lose support for this strategy in light of the results of this study. As the risk is more unpredictable, the reliance on herd immunity becomes less supportable, and the need for individual protective actions more pressing. Thus, the results of this study provide support for education and prevention interventions that target the risk populations on an ongoing basis, rather than ahead of a seasonal or periodic epidemic. These evidence-supported interventions will 
yield significant reductions in the morbidity and mortality associated with IPD, and the positive social change that results from reductions in suffering and societal cost.

The efficacy of pneumococcal vaccines, and vaccine coverage of the most common virulent serotypes continues to be an issue of public health importance (Jackson et al., 2003; Madhi, Whitney, \& Nohynek; Maki, 2004; McDaniel \& Swialto, 2004). Assessment of vaccine efficacy and changes that might be required in vaccine serotype coverage is improved by a better understanding of the epidemic dynamics of IPD. It could have been argued that administration of vaccine at opposing points in an epidemic cycle (administration in the "trough" of the dynamic, and assessment of efficacy at the peak), could give a false impression of poor vaccine efficacy, when the increased incidence of IPD was part of a predictable natural phenomenon. As no periodicity appears to exist, this can be excluded as a factor in vaccine efficacy, and researchers can target other areas impacting vaccine efficacy, such as molecular factors (Obert et al., 2006).

\section{Recommendations for Action}

The recommendations for action as a result of this study are two-fold. First, the absence of a periodic dynamic of IPD epidemics must be shared with the many stakeholders concerned with IPD prevention and treatment. Publication in a peerreviewed journal, as well as presentations of the results to appropriate professional audiences is the minimum action in this regard. Second, the use of time series analysis as a tool for examining disease dynamics should be similarly described. While a substantial body of literature addresses the subject, the application of these techniques is not a standard tool in epidemiologic research. These techniques are complex, but can enhance 
our understanding of disease dynamics, which warrants further discussion and constructive criticism of the processes.

\section{Recommendations for Future Studies}

The use of frequency domain time-series analytic techniques for epidemiologic research, as applied in this study, is supported by the literature; however, future studies of IPD epidemic patterns might benefit from a variation of time-series analysis, waveletbased incidence analysis. A system exhibiting a chaotic dynamic, as found in this study, would be well suited to the wavelet analysis technique (Cazelles, Chavez, Berteaux, Menard, Vik, Jenouvrier, et al., 2008). Wavelet analysis techniques have value in understanding aperiodic epidemics (Cazelles et al., 2008), which it appears may be the dynamic of IPD. Thus, it is recommended that a future study of IPD dynamics using this technique be applied to a suitable data set.

An additional study might apply either frequency domain time-series analysis or wavelet analysis to a different data set, that of the Active Bacterial Core Surveillance system. It was this data set that revealed seasonality; however, it is smaller in time span than the NHDS. Time-series analysis requires large data sets, and the limited data set size might significantly reduce the ability to detect periodicity in addition to seasonality.

\section{Conclusion}

This study set out to determine whether invasive infection by $S$. pneumoniae in the United States occurs in an epidemic pattern of a predictable recurrent nature and definable frequency. Although annual cyclic increases have been reported in the literature, little was known about IPD epidemic patterns for larger periods of time, limiting our ability to detect changes in IPD epidemic dynamics and thus to predict 
outbreaks. This study attempted to address this gap in the knowledge in the epidemiology of IPD through a retrospective cohort design using a population-based cohort from the National Hospital Discharge Survey conducted from 1979-2006. Drawing from the theoretical base of dynamic modeling of stochastic epidemic systems, this study utilized time-series methods to examine IPD epidemic dynamics. The results of the study were consistent with the finding that IPD in the United States, for the period from 1979-2006, did not occur in periodic epidemics of a definable frequency outside of the predicted seasonal increase in incidence.

The results of this study provide support for maintaining established evidence based surveillance practices and current methods of disease prevention. This in turn could significantly reduce the public health impact of IPD by reducing the number of hospitalizations, and decreasing morbidity and mortality. This promotes positive social change through improved of health outcomes in the general population and decreased health care costs (Ament et al., 2000). 


\section{References}

Abdeldaim, G. M. K., Stralin, K., Olcen, P., Blomberg, J., \& Herrmann, B. (2008). Toward a quantitative DNA-based definition of pneumococcal pneumonia: A comparison of Streptococcus pneumoniae target genes, with special reference to the Spn9802 fragment. Diagnostic Microbiology and Infectious Disease, 60, 143150 .

Akduman, D., Ehret, J. M., \& Judson, F. N. (2006). Comparison of secular trends in pneumococcal serotypes causing invasive disease in Denver, Colorado (19712004) and serotype coverage by marketed pneumococcal vaccines. Clinical Microbiology and Infection, 12, 1131-1146.

Alanee, S. R., McGee, L., Jackson, D., Chiou, C. C., Feldman, C., \& Morris, A. J. (2007). Association of serotypes of Streptococcus pneumoniae with disease severity and outcome in adults: An international study. Clinical Infectious Diseases, 45(46$51)$.

Alekshun, M. N., \& Levy, S. B. (2006). Commensals upon us. Biochemical Pharmacology, 71(893-900).

Alexander, M. E., Moghadas, S. M., Rohani, P., \& Summers, A. R. (2006). Modeling the effect of a booster vaccination on disease epidemiology. Journal of Mathematical Biology, 52, 290-306.

Allegrucci, M., \& Sauer, K. (2007). Characterization of colony morphology variants isolated from Streptococcus pneumoniae biofilms. Journal of Bacteriology, 189, 2030-2038.

Altizer, S., Dobson, A., Hosseini, P., Hudson, P., Pascual, M., \& Rohani, P. (2006). Seasonality and the dynamics of infectious diseases. Ecology Letters, 9, 467-494.

Amber, I. J., Gilbert, E. M., Schiffman, G., \& Jacobson, J. A. (1990). Increased risk of pneumococcal infections in cardiac transplant recipients. Transplantation, 49, 122-125.

Ament, A., Baltussen, R., Duru, G., Rigaud-Bully, C., de Graeve, D., Christie, P., et al. (2000). Cost-effectiveness of pneumococcal vaccination of older people: A study in 5 western European countries. Clinical Infectious Diseases, 31, 444-450.

American National Standards Institute (2002). Information Systems - Coded Character Sets - 7-Bit American National Standard Code for Information Interchange (7-Bit ASCII) (ANSI INCITS 4-1986 (R2002)). New York: American National Standards Institute. 
American Public Health Association (2000). Pneumococcal Pneumonia. In J. Chin (Ed.), Control of Communicable Diseases Manual (17th ed., p. 388). Washington, DC: American Public Health Association.

Ampofo, K., Bender, J., Sheng, X., Korgenski, K., Daly, J., Pavia, A. T., et al. (2008). Seasonal invasive pneumococcal disease in children: Role of preceding respiratory viral infections. Pediatrics, 122, 229-237.

Anderson, R. M., Grenfell, B. T., \& May, R. M. (1984). Oscillatory fluctuations in the incidence of infectious disease and the impact of vaccination: Time series analysis. Journal of Hygiene, 93, 587-608.

Andonegui, G., Goring, K., LIu, D., McCafferty, D.-M., \& Winston, B. W. (2009). Characterization of S. pneumoniae-induced multiple organ dysfunction syndrome: An experimental mouse model of gram-positive sepsis. Shock, 31, 423-428.

Aron, J. L. (2007). Mathematical modeling: The dynamics of infection. In K. E. Nelson \& C. M. Williams (Eds.), Infectious Disease Epidemiology-Theory and Practice (2nd ed., pp. 181-209). Boston: Jones and Bartlett.

Austrian, R. (1954). Morphologic variations in pneumococcus. Part I. An analysis of the bases for morphologic variations in pneumococcus and description of a hitherto undefined morphologic variant. Journal of Experimental Medicine, 98, 21-37.

Austrian, R. (1957). The syndrome of pneumococcal endocarditis, meningitis and rupture of the aortic valve. Transactions of the American Clinical and Climatological Association, 68, 40-50.

Austrian, R. (1960). Some contributions of pneumococcal genetics to the elucidation of biological phenomena. Bulletin of the New York Academy of Medicine, 36, 355363.

Austrian, R. (1960). The Gram stain and the etiology of lobar pneumonia, an historical note. Bacteriology Review, 24, 261-265.

Austrian, R. (1978). The Jeremiah Metzger Lecture: Of gold and pneumococci: A history of pneumococcal vaccines in South Africa. Transactions of the American Clinical and Climatological Association, 89, 141-161.

Austrian, R. (1981). Pneumococcus: The first one hundred years. Review of Infectious Disease, 3, 183-189.

Austrian, R. (1999a). Oswald T. Avery: the wizard of York Avenue. American Journal of Medicine, 107, 7S-11S. 
Austrian, R. (1999b). Pneumococcus and the Brooklyn connection. American Journal of Medicine, 107, 2S-6S.

Austrian, R. (2000). The enduring pneumococcus: Unfinished business and opportunities for the future. In A. Tomasz (Ed.), Streptococcus pneumoniae: Molecular Biology \& Mechanisms of Disease (pp. 3-7). Larchmont, NY: Mary Ann Liebert.

Austrian, R. (2004). Forward. In E. I. Tuomanen, T. J. Mitchell, D. Morrison \& B. G. Spratt (Eds.), The Pneumococcus (pp. xv-xxvii). Washington, DC: ASM Press.

Avery, O. T., \& Dubos, R. (1931). The protective action of a specific enzyme against Type III pneumococcus infection in mice. Journal of Experimental Medicine, 54, 73-90.

Avery, O. T., MacLeod, C. M., \& McCarty, M. (1944). Studies on the chemical nature of the substance inducing transformation of pneumococcal types. Journal of Experimental Medicine, 79, 137-158.

Babbie, E. (2007). Experiments. In: The Practice of Social Research (11th ed., pp. 221242). Belmont, CA: Thomson Wadsworth.

Babl, F. E., Pelton, S. I., Theodore, S., \& Klein, J. O. (2000). Constancy of distribution of serogroups of invasive pneumococcal isolates among children: Experience during 4 decades. Clinical Infectious Diseases, 32, 1155-1161.

Balakrishnan, I., Crook, P., Morris, R., \& Gillespie, S. H. (2000). Early predictors of mortality in pneumococcal bacteremia. Journal of Infection, 40, 256-261.

Ball, P. (1999). Therapy for pneumococcal infection at the millennium: Doubts and uncertainties. American Journal of Medicine, 107, 77S-85S.

Bansal, S., Grenfell, B. T., \& Meyers, L. A. (2007). When individual behaviour matters: Homogeneous and network models in epidemiology. Journal of the Royal Society Interface, 4, 879-801.

Barocchi, M. A., Ries, J., Zogaj, X., Hemsley, C., Albiger, B., Kanth, A., et al. (2006). A pneumococcal pilus influences virulence and host inflammatory responses. Proceedings of the National Academy of Sciences of the United States of America, 103, 2857-2962.

Bauch, C. T. (2005). Imitation dynamics predict vaccinating behaviour. Proceedings of the Royal Society 272, 1669-1675.

Bauch, C. T., \& Earn, D. J. D. (2003). Transients and attractors in epidemics. Proceedings: Biological Sciences, 270, 1573-1578. 
Bauch, C. T., \& Earn, D. J. D. (2004). Vaccination and the theory of games. Proceedings of the National Academy of Sciences of the United States of America, 101, 1339113394.

Beadsworth, M. B. J., Wooton, D., Chenzbraun, A., \& Beeching, N. J. (2007). Austrian's syndrome: The first described case of pneumococcal meningitis pneumonia and endocarditits in an injecting drug user. European Journal of Internal Medicine, 18, 605-606.

Beall, B., McEllistrem, M. C., Gertz, R. E., Boxrud, D. J., Besser, J. M., Harrison, L. H., et al. (2002). Emergence of a novel penicillin-nonsusceptible, invasive serotype 35B clone of Streptococcus pneumoniae within the United States. Journal of Infectious Diseases, 186, 118-122.

Bean, B., \& Tomasz, A. (1973). 5-Flouropyrimidine-resistant mutants of pneumococcus. Journal of Bacteriology, 113, 1348-1355.

Benin, A. L., O'Brien, K. L., Watt, J. P., Reid, R., Zell, E. R., Katz, S., et al. (2003). Effectiveness of the 23-Valent Polysaccharide Vaccine against Invasive Pneumococcal Disease in Navajo Adults. The Journal of Infectious Diseases, 188(1), 81-89.

Berk, S. L., Gage, K. A., Holtsclaw-Berk, S. A., \& Smith, J. K. (1985). Type 8 pneumococcal pneumonia: An outbreak on an oncology ward. Southern Medical Journal, 78, 159-161.

Berg, S., Trollfors, B., Persson, E., Backhaus, E., Larsson, P., Ek, E., et al. (2006). Serotypes of Streptococcus pneumoniae isolated from blood and cerebrospinal fluid related to vaccine serotypes and to clinical characteristics. Scandanavian Journal of Infectious Diseases, 38, 427-432.

Bernatoniene, J., \& Finn, A. (2005). Advances in pneumococcal vaccines-Advantages for infants and children. Drugs, 69, 229-255.

Betts, R. F. (2003). Upper Respiratory Tract Infections. In R. Betts, S. Chapman \& R. Penn (Eds.), Reese and Betts' A Practical Approach to Infectious Diseases (5th ed., pp. 251-268). Philadelphia: Lippincott Williams \& Wilkins.

Bjornstad, O. N., Finkenstadt, B. F., \& Grenfell, B. T. (2002). Dynamics of measles epidemics: Estimating scaling of transmission rates using a time series SIR model. Ecological Monographs, 72, 169-184. 
Black, S., \& Shinefield, H. (2002). Safety and efficacy of the seven-valent pneumococcal conjugate vaccine: Evidence from northern California. European Journal of Pediatrics, 161, S127-S131.

Bloom, H. G., Wheeler, D. A., \& Lynn, J. (1999). A managed care organization's attempt to increase influenza and pneumococcal immunizations for older adults in an acute care setting. Journal of the American Geriatrics Society, 47, 106-110.

Bolker, B. M., \& Grenfell, B. T. (1996). Impact of vaccination on the spatial correlation and persistence of measles dynamics. Proceedings of the National Academy of Sciences of the United States of America, 93, 12648-12653.

Boots, M., Greenman, J., Ross, D., Norman, R., Hails, R., \& Sait, S. (2003). The population dynamical implications of covert infections in host-microparasite interactions. Journal of Animal Ecology, 72, 1064-1072.

Bootsma, H. J., Egmont-Peterson, M., \& Hermans, P. W. M. (2007). Analysis of the in vitro transcriptional response of human pharyngeal epithelial cells to adherent Streptococcus pneumoniae: Evidence for a distinct response to encapsulated strains. Infection and Immunity, 75, 5489-5499.

Borer, A., Meirson, H., Peled, N., Porat, N., Dagan, R., Fraser, D., et al. (2001). Antibiotic-resistant pneumococci carried by young children do not appear to disseminate to adult members of a closed community. Clinical Infectious Diseases, 33, 436-444.

Boulet, S. L., Correa-Villasenor, A., Hsia, J., \& Atrash, H. (2006). Feasibility of using the National Hospital Discharge Survey to estimate the prevalence of selected birth defects. Birth Defects Research (Part A): Clinical and Molecular Teratology, 76, 757-761.

Bouza, E., Pintado, V., Rivera, S., Blazquez, R., Munoz, P., Cercenado, E., et al. (2005). Nosocomial bloodstream infections caused by Streptococcus pneumoniae. Clinical Microbiology and Infection, 11, 919-924.

Bowers, E. F., \& Jeffries, L. R. (1955). Optochin in the identification of Str. pneumoniae. Journal of Clinical Pathology, 8, 58-60.

Breiman, R. F., Keller, D. W., Phelan, M. A., Sniadak, D. H., Stevens, D. S., Rimland, D., et al. (2000). Evaluation of effectiveness of the 23-valent pneumococcal capsular polysaccharide vaccine for HIV-infected patients. Archives of Internal Medicine, 160, 2633-2638. 
Bridy-Pappas, A. E., Margolis, M. B., Center, K. J., \& Isaacman, D. J. (2005).

Streptococcus pneumoniae: Description of the pathogen, disease epidemiology, treatment, and prevention. Pharmacotherapy, 190, 1193-1211.

Briles, D. E., Novak, L., Hotomi, M., Ginkel, F. W. v., \& King, J. (2005). Nasal colonization with Streptococcus pneumoniae includes subpopulations of surface and invasive pneumococci. Infection and Immunity, 73, 6945-6951.

Broutin, H., Guegan, J.-F., Elguero, E., Simondon, F., \& Cazelles, B. (2005). Large-scale comparative analysis of Pertussis population dynamics: Periodicity, synchrony, and impact of vaccination. American Journal of Epidemiology, 161(12), 11591167.

Brueggemann, A. B., Griffiths, D. T., Meats, E., Peto, T., Crook, D. W., \& Spratt, B. G. (2003). Clonal relationships between invasive and carriage Streptococcus pneumoniae and serotype- and clone-specific differences in invasive disease potential. Journal of Infectious Diseases(1424-1432).

Brueggemann, A. B., Peto, T. E., Crook, D. W., Butler, J. C., \& Kristinsson, K. G. (2004). Temporal and geographic stability of the serogroup-specific invasive disease potential of Streptococcus pneumoniae in children. Journal of Infectious Diseases, 190, 1203-1211.

Brueggemann, A. B., Pfaller, M. A., \& Doern, G. V. (2001). Use of penicillin MICs to predict in vitro activity of other beta-lactam antimicrobial agents against Streptococcus pneumoniae. Journal of Clinical Microbiology, 39, 367-369.

Brueggemann, A. B., \& Spratt, B. G. (2003). Geographic distribution and clonal diversity of Streptococcus pneumoniae serotype 1 isolates. Journal of Clinical Microbiology, 41, 4966-4970.

Buie, K. A., Klugman, K. P., Gottberg, A. v., Perovic, O., Karstaedt, A., Crewe-Brown, H. H., et al. (2004). Gender as a risk factor for both antibiotic resistance and infection with pediatric serogroups/serotypes, in HIV-infected and -uninfected adults with pneumococcal bacteremia. Journal of Infectious Diseases, 189, 19962000 .

Butler, J. C. (2004). Epidemiology of pneumococcal disease. In E. I. Tuomanen, T. J. Mitchell, D. A. Morrison \& B. G. Spratt (Eds.), The Pneumococcus (pp. 148168). Washington, DC: ASM Press.

Butler, J. C., \& Cetron, M. S. (1999). Pneumococcal drug resistance: the new "Special enemy of old age". Clinical Infectious Diseases, 28, 730-735. 
Butler, J. C., Crengle, S., Cheek, J. E., Leach, A. J., Lennon, D., O'Brien, K. L., et al. (2001). Emerging infectious diseases among indigenous peoples. Emerging Infectious Diseases, 7, 554-556.

Butler, J. C., Dowell, S. F., \& Breiman, R. F. (1998). Epidemiology of emerging pneumococcal drug resistance: Implications for treatment and prevention. Vaccine, 18, 1693-1697.

Butler, J. C., \& Schuchat, A. (1999). Epidemiology of pneumococcal infections in the elderly. Drugs and Aging, 15, 11-19.

Butler, J. C., Shapiro, E. D., \& Carlone, G. M. (1999). Pneumococcal vaccines: History, current status, and future directions. American Journal of Medicine, 107, 69S$77 \mathrm{~S}$.

Cabellos, C., Verdaguer, R., Olmo, M., Fernandez-Sabe, N., Cisnal, M., Ariza, J., et al. (2009). Community-acquired bacterial menigitis in elderly patients. Medicine, 88 , 115-119.

Campos, I. B., Darrieux, M., Ferreira, D. M., Mihaji, E. N., Silva, D. A., Areas, A. P. M., et al. (2008). Nasal immunization of mice with Lactobacillus casei expressing the pneumococcal surface protein A: Induction of antibodies, complement deposition and partial protection against Streptococcus pneumoniae challenge. Microbes and Infection, 10, 481-488

Cao, J., Li, D., Gong, Y., Yin, N., Chen, T., Wong, C. K., et al. (2009). Caseinolytic protease: A protein vaccine which could elicit serotype-independent protection against invasive pneumococcal infection. Clinical and Experimental Immunology, $156,52-60$.

Carrolo, M., Pinto, F. R., Melo-Cristino, J., \& Ramirez, M. (2009). Pherotypes are driving genetic differentiation within Streptococcus pneumoniae. BMC Microbiology, 9, 1-10.

Castillo, E. M., Rickman, L. S., Brodine, S. K., Ledbetter, E. K., \& Kelly, C. (2000). Streptococcus pneumoniae: Bacteremia in an era of penicillin resistance. American Journal of Infection Control, 28, 239-243.

Cazelles, B., Chavez, M., Berteaux, D., Menard, F., Vik, J. O., Jenouvrier, S., et al. (2008). Wavelet analysis of ecological time series. Oecologia, 156, 287-304.

Centers for Disease Control and Prevention (2006). Pneumococcal Disease. In Epidemiology and Prevention of Vaccine-Preventable Diseases (6th ed., pp. 256257). Atlanta, GA: Centers for Disease Control and Prevention. 
Centers for Disease Control and Prevention. (2007a). SAS_INPUT_NHDS_PUFILE.

Retrieved November 4, 2008, from

ftp://ftp.cdc.gov/pub/Health_Statistics/NCHS/Dataset_Documentation/NHDS/SA S_INPUT_NHDS_PUFILE.txt

Centers for Disease Control and Prevention (2007b). Streptococcus pneumoniae, drugresistant invasive disease (DRSP)-2007 case definition. Retrieved August 15, 2007, from http://www.cdc.gov/epo/dphsi/casedef/streptococcuscurrent.htm

Chapman, S. J., Khor, C. C., Vanberg, F. O., Frodsham, A., Walley, A., Maskell, N. A., et al. (2007). IkB genetic polymorphisms and, Invasive Pneumococcal Disease. American Journal of Respiratory and Critical Care Medicine, 176, 181-187.

Chen, W.-Y., \& Bokka, S. (2005). Stochastic modeling of nonlinear epidemiology. Journal of Theoretical Biology, 234, 455-470.

Chen, F. M., Breiman, R. F., Farley, M., Plikaytis, B., K.Deaver, \& Cetron, M. S. (1998). Geocoding and linking data from population-based surveillance and the US census to evaluate the impact of median household income on the epidemiology of invasive Streptococcus pneumoniae infections. American Journal of Epidemiology, 148, 1212-1218.

Chin, J. (2000). Conjunctivitis/Keratitis. In J. Chin \& M. Ascher (Eds.), Control of Communicable Diseases Manual (17th ed., pp. 119-121). Washington, DC: American Public Health Association.

Christenson, B., Lundbergh, P., Hedlund, J., \& Ortqvist, A. (2001). Effects of a largescale intervention with influenza and 23 -valent pneumococcal vaccines in adults aged 65 years or older: A prospective study. Lancet, 357, 1008-1011.

Cooley, J. W., \& Tukey, J. W. (1965). An algorithm for the machine calculation of complex Fourier series. Mathematics of Computation, 19, 297-301.

Crewe-Brown, H. H., Karstaedt, A. S., Saunders, G. L., Khoosal, M., Jones, N., Wasas, A., et al. (1997). Streptococcus pneumoniae blood culture isolates from patients with and without Human Immunodeficiency Virus infection: Alterations in penicillin susceptibilities and in serogroups and serotypes. Clinical Infectious Diseases, 25, 1165-1172.

Crook, D. W. (2006). Capsular type and the pneumococcal human host-parasite relationship. Clinical Infectious Diseases, 42, 460-462.

Cundell, D. R., Weiser, J. N., Shen, J., Young, A., \& Tuomanen, E. I. (1995). Relationship between colonial morphology and adherence of Streptococcus pneumoniae. Infection and Immunity, 63, 757-761. 
Dagan, R., Givon-Lavi, N., Zamir, O., Sikuler-Cohen, M., Guy, L., Janco, J., et al. (2002). Reduction of nasopharyngeal carriage of Streptococcus pneumoniae after administration of a 9-valent pneumococcal conjugate vaccine to toddlers attending day care centers. Journal of Infectious Diseases, 185, 927-936.

Dagan, R., \& Lipsitch, M. (2004). Changing the ecology of pneumococci with antibiotics and vaccines. In E. I. Tuomanen, T. J. Mitchell, D. A. Morrison \& B. G. Spratt (Eds.), The Pneumococcus (pp. 283-313). Washington, DC.: ASM Press.

Danai, P. A., Sinha, S., Moss, M., Haber, M. J., \& Martin, G. S. (2007). Seasonal variation in the epidemiology of sepsis. Critical Care Medicine, 35, 410-415.

Daneman, N., McGeer, A., Green, K., \& Low, D. E. (2006). Macrolide resistance in bacteremic pneumococcal disease: Implications for patient management. Clinical Infectious Diseases, 43, 432-438.

Daum, R. S., Hogerman, D., Rennels, M. B., Bewley, K., Malinoski, F., Rothstein, E., et al. (1997). Infant immunization with pneumococcal CRM197 vaccines: Effect of saccharide size on immunogenicity and interactions with simultaneously administered vaccines. Journal of Infectious Diseases, 176, 445-455.

Davidson, M., Schraer, C. D., Parkinson, A. J., Campbell, J. F., Facklam, R. R., Wainwright, R. B., et al. (1989). Invasive pneumococcal disease in an Alaska native population, 1980 through 1986. JAMA, 261(5), 715-718.

De Bruin, M. L., van Hemel, N. M., Leufkens, H. G. M., \& Hoes, A. W. (2005). Hospital discharge diagnoses of ventricular arrhythmias and cardiac arrest were useful for epidemiologic research. Journal of Clinical Epidemiology, 58, 1325-1329.

De Coster, C., Quan, H., Finlayson, A., Gao, M., Halfon, P., Humphries, K. H., et al. (2006). Identifying priorities in methodological research using ICD-9-CM and ICD-10 administrative data: report from an international consortium. BMC Health Services Research, 6, 1-6. Retrieved August 6, 2008, from http://www.biomedcentral.com/1472-6963-6-77

Demlo, L. K., \& Campbell, P. M. (1981). Improving hospital discharge data: Lessons from the National Hospital Discharge Survey. Medical Care, 19, 1030-1040.

Dennison, C., \& Pokras, R. (2000). Design and operation of the National Hospital Discharge Survey: 1988 redesign. Vital and Health Statistics, Series 1: Programs and Collection Procedures, 39, 1-13. 
De Vecchi, E., Nicola, L., Ossola, F., \& Drago, L. (2009). In vitro selection of resistance in Streptococcus pneumoniae at in vivo fluoroquinolone concentrations. Journal of Antimicrobial Chemotherapy, 63, 721-727.

D'Innocenzo, A., Paladini, F., \& Renna, L. (2006). A numerical investigation of discrete oscillating epidemic models. Physica A, 364, 497-512.

Dochez, A. R., \& Avery, O. T. (1915). Varieties of pneumococcus and their relation to lobar pneumonia. Journal of Experimental Medicine, 114-132.

Dopazo, J., Mendoza, A., Herrero, J., Caldara, F., Humbert, Y., Friedli, L., et al. (2001). Annotated draft genomic sequence from a Streptococcus pneumoniae type 19F clinical isolate. Microbial Drug Resistance-Mechanisms Epidemiology \& Disease, 7, 99-125.

Dorman, N. J. (2003). Sepsis. In R. Betts, S. Chapman \& R. Penn (Eds.), A Practical Approach to Infectious Disease (5th ed., pp. 19-66). Philadelphia: Lippincott Williams and Wilkins.

Dowdle, W. R. (2006). Influenza pandemic periodicity, virus recycling, and the art of risk assessment. Emerging Infectious Diseases, 12, 34-39.

Dowell, S. F. (2001). Seasonal variation in host susceptibility and cycles of certain infectious diseases. Emerging Infectious Diseases, 7, 369-374.

Dowell, S. F., Whitney, C. G., Wright, C., Rose, C. E., \& Schuchatt, A. (2003). Seasonal patterns of invasive pneumococcal disease. Emerging Infectious Diseases, 9, 573 579.

Dowson, C. G., Coffey, T. J., \& Spratt, B. G. (1994). Origin and molecular epidemiology of penicillin-binding-protein-mediated resistance to beta-lactam antibiotics. Trends in Microbiology, 2, 361-366.

Duncan, C. J., Duncan, S. R., \& Scott, S. (1996). Whooping cough epidemics in London, 1701-1812: Infection dynamics, seasonal forcing, and the effects of malnutrition. Proceedings of the Royal Society, 263, 445-450.

Duppenthaler, A., Gorgievski-Hrisoho, M., Frey, U., \& Aebi, C. (2003). Two-year periodicity of Respiratory Syncytial Virus epidemics in Switzerland. Infection, 31, 75-80.

Dworkin, M. S., Ward, J. W., Hanson, D. L., Jones, J. L., \& Kaplan, J. E. (2001). Pneumococcal disease among Human Immunodeficiency Virus-infected persons: Incidence, risk factors, and impact of vaccination. Clinical Infectious Diseases, $32,794-800$. 
Earn, D. J. D., Rohani, P., Bolker, B. M., \& Grenfell, B. T. (2000). A simple model for complex dynamical transitions in epidemics. Science, 287, 667-670.

Ellner, S. P., Bailey, B. A., Bobashev, G. V., Gallant, A. R., Grenfell, B. T., \& Nychka, D. W. (1998). Noise and nonlinearity in measles epidemics: Combining mechanistic and statistical approaches to population modeling. American Naturalist, 151, 425-440.

Fedson, D. S., Harward, M. P., Reid, R. A., \& Kaiser, D. L. (1990). Hospital-based pneumococcal immunization: Epidemiological rationale from the Shenandoah study. Journal of the American Medical Association, 264, 1117-1122.

Feikin, D. R., Elie, C. M., Goetz, M. B., Lennox, J. L., Carlone, G. M., Romero-Steiner, S., et al. (2004). Specificity of the antibody response to the pneumococcal polysaccharide and conjugate vaccines in Human Immunodeficiency Virusinfected adults. Clinical and Diagnostic Laboratory Immunology, 11, 137-141.

Fine, P. E. (1993). Herd immunity: history, theory, practice. Epidemiologic Reviews, 15, 265-302.

Finkenstaedt, B. F., Bjornstad, O. N., \& Grenfell, B. T. (2002). A stochastic model for extinction and recurrence of epidemics: Estimation and inference for measles outbreaks. Biostatistics, 3, 493-510.

Finkenstadt, B. F., \& Grenfell, B. T. (2000). Time series modeling of childhood diseases: A dynamical systems approach. Applied Statistics, 49, 187-205.

Fisman, D. N. (2007). Seasonality of infectious diseases. Annual Review of Public Health, 28, 127-143.

Francq, C., \& Roussignol, M. (1997). On white noises driven by hidden Markov chains. Journal of Time Series Analysis, 18, 553-578.

Galanis, E., King, A. S., Vaughese, P., \& Halperin, S. A. (2006). Changing epidemiology and emerging risk groups for pertussis. Canadian Medical Association Journal, $174,451-452$.

Garau, G., Lemaire, D., Vernet, T., Dideberg, O., \& Guilmi, A. M. D. (2005). Crystal structure of phosphorylcholine esterase domain of the virulence factor cholinebinding protein E from Streptococcus pneumoniae. Journal of Biological Chemistry, 280, 28591-28600.

Gnedenko, B. V. (1962). Markov Chains (B. D. Seckler, Trans.). In The Theory of Probability (pp. 125-144). New York: Chelsea Publishing. 
Gertz, R.E., McEllistrem, M. C., Boxrud, D. J., Li, Z., Sakota, V., Thompson, T. A., et al. (2003). Clonal distribution of invasive pneumococcal isolates from children and selected adults in the United States prior to 7-valent conjugate vaccine introduction. Journal of Clinical Microbiology, 41, 4194-4216.

Gilbert, B., Menetrey, C., Blin, V., Brosset, P., Lumley, L. d., \& Fisher, A. (2002). Familial isolated congenital asplenia: A rare, frequently hereditary dominant condition, often detected too late as a cause of overwhelming pneumococcal sepsis. Report of a new case and review of 31 others. European Journal of Pediatrics, 161, 368-372.

Goldstein, E. J. C., \& Garabedian-Ruffalo, S. M. (2002). Widespread use of fluoroquinolones versus emerging resistance in pneumococci. Clinical Infectious Diseases, 35(1505-1511).

Gordon, S. B., \& French, N. (2005). Should we develop an inhaled anti-pneumococcal vaccine for adults. Current Medical Chemotherapy and Anti-Infective Agents, 4, 75-89.

Grassly, N. C., \& Fraser, C. (2006). Seasonal infectious disease epidemiology. Proceedings of the Royal Society, 273, 2541-2550.

Gray, B. M. (2000). Streptococcus pneumoniae infections. In D. Stevens \& E. Kaplan (Eds.), Streptococcal Infections-Clinical Aspects, Microbiology, and Molecular Pathogenesis (pp. 302-332). New York: Oxford University Press.

Greene, G. S., \& Demasi, R. (1996). Penicillin-Resistant Pneumococcal Meningitis: Navigating a Therapeutic Minefield. The American Journal of Medical Sciences, 311(4), 180-185.

Greenwood, B. (1999). The epidemiology of pneumococcal infection in children in the developing world. Philosophical Transactions: Biological Sciences, 354, 777785.

Grenfell, B. T., Bjornstad, O. N., \& Finkenstadt, B. F. (2002). Dynamics of measles epidemics: Scaling noise, determinism, and predictability with the TSIR model. Ecological Monographs, 72, 185-202.

Grenfell, B. T., Bjornstad, O. N., \& Kappy, J. (2001). Traveling waves and spatial hierarchies in measles epidemics. Nature, 414, 716-723.

Grenfell, B. T., Pybus, O. G., Gog, J. R., Wood, J. L. N., Daly, J. M., Mumford, J. A., et al. (2004). Unifying the epidemiological and evolutionary dynamics of pathogens. Science, 303, 327-332. 
Grijalva, C. G., Nuorti, J. P., Arbogast, P. G., Martin, S. W., Edwards, K. M., \& Griffin, M. R. (2007). Decline in pneumonia admissions after routine childhood immunization with pneumococcal conjugate vaccine in the USA: A time series analysis. Lancet, 369, 1179-1186.

Guchev, I. A., Yu, V. L., Sinopalnikov, A., Klochkov, O. I., Koslov, R. S., \& Stratchnounski, L. S. (2005). Management of nonsevere pneumonia in military trainees with the urinary antigen test for Streptococcus pneumoniae: An innovative approach to targeted therapy. Clinical Infectious Diseases, 40, 16081616.

Guevara, R. E., Butler, J. C., Marston, B. J., Plouffe, J. F., File, T. M., \& Breiman, R. F. (1999). Accuracy of ICD-9CM codes in detecting community-acquired pneumococcal pneumonia for incidence and vaccine efficacy studies. American Journal of Epidemiology, 149, 282-289.

Gundel, M., \& Schwarz, F. K. T. (1932). Studien uber die Bakterienflora der oberen Atmungswege Neugeborener (im Vergleich der Mundholenflora der Mutter und des Pflegepersonals) unter besonder Berucksichtigung ihrer Bedeutung fur das Pneumonieproblem. Zeitschrift für Hygiene und Infektionskrankheiten, 113, 411436.

Guris, D., Strebel, P. M., Bardenheir, B., Brennan, M., Tachdjian, R., Finch, E., et al. (1999). Changing epidemiology of Pertussis in the United States: Increasing reported incidence among adolescents and adults, 1990-1996. Clinical Infectious Diseases, 28, 1230-1237.

Haber, M., Barskey, A., Baughman, W., Barker, L., Whitney, C. G., Shaw, K. M., et al. (2005). Herd immunity and pneumococcal conjugate vaccine: A quantitative model. Vaccine, 25, 5390-5398.

Haddad, M. B., Porucznik, C. A., Joyce, K. E., De, A. K., Pavia, A. T., Rolfs, R. T., et al. (2008). Risk factors for pediatric invasive pneumococcal disease in the intermountain west, 1996-2002. Annals of Epidemiology, 18, 139-146.

Hagenaars, T. J., Donnelly, C. A., \& Ferguson, N. M. (2004). Spatial heterogeneity and the persistence of infectious diseases. Journal of Theoretical Biology, 229, 349359.

Hakenbeck, R., Balmelle, N., Weber, B., Gardes, C., Keck, W., \& Saizieu, A. D. (2001). Mosaic genes and mosaic chromosomes: Intra- and interspecies genomic variation of Streptococcus pneumoniae. Infection and Immunity, 69, 2477-2486. 
Hamaker, E. L., Dolan, C. V., \& Molenaar, P. C. M. (2005). Statistical modeling of the individual: Rationale and application of multivariate stationary time series analysis. Multivariate Behavioral Research, 40, 207-233.

Hamdad, F., Canarelli, B., Rousseau, F., Thomas, D., Biendo, M., Eb, F., et al. (2007). Les menigites a Streptococcus pneumoniae au CHU d'Amiens de 1990 a 2005. Aspects bacteriologiques des souches isolees. Pathologie Biologie, 55, 446-452.

Hammitt, L. L., Bruden, D. L., Butler, J. C., Baggett, H. C., Hurlburt, D. A., Reasonover, A., et al. (2006). Indirect effect of conjugate vaccine on adult carriage of Streptococcus pneumoniae: An explanation of trends in invasive pneumococcal disease. Journal of Infectious Diseases, 193, 1487-1494.

Hanage, W. P., Huang, S. S., Lipsitch, M., Bishop, C. J., Godoy, D., Pelton, S. I., et al. (2007). Diversity and antibiotic resistance among nonvaccine serotypes of Streptococcus pneumoniae carriage isolates in the post-heptavalent conjugate vaccine era. Journal of Infectious Diseases, 195, 347-352.

Harrison, L. H., Dwyer, D. M., Billman, L., Kolczak, M. S., \& Schuchat, A. (2000). Invasive Pneumococcal Infection in Baltimore, Md: Implications for Immunization Policy. Archives of Internal Medicine, 160(1), 89-94.

Hausdorff, W. P. (2007). The roles of pneumococcal serotypes 1 and 5 in paediatric invasive disease. Vaccine, 25, 2406-2413.

Hausdorff, W. P., Bryant, J., Kloek, C., Paradiso, P. R., \& Siber, G. R. (2000). The contribution of specific pneumococcal serogroups to different disease manifestations: Implications for conjugate vaccine formulation and use, part II. Clinical Infectious Diseases, 30, 122-140.

Hausdorff, W. P., Feikin, D. R., \& Klugman, K. P. (2005). Epidemiological differences among pneumococcal serotypes. Lancet Infectious Diseases, 5, 83-93.

Hausdorff, W. P., Silber, G., \& Paradiso, P. R. (2001). Geographical differences in invasive pneumococcal disease rates and serotype frequency in young children. Lancet, 357, 950-952.

Hawley, L. A., \& Whitney, C. G. (2002). Pneumococcal Diseases. In Vaccine Preventable Diseases Surveillance Manual (3rd ed., pp. 9-1-9-4). Atlanta, GA: Centers for Disease Control and Prevention.

Heffernan, R. T., Barrett, N. L., Gallagher, K. M., Halder, J. L., Harrison, L. H., Reingold, A. L., et al. (2005). Declining incidence of invasive Streptococcus pneumoniae infections among persons with AIDS in an era of highly active retroviral therapy, 1995-2000. Journal of Infectious Diseases, 191, 2038-2045. 
Hennessy, T. W., Peterson, K. M., Bruden, D., Parkinson, A. J., Hurlburt, D., Getty, M., et al. (2002). Changes in antibiotic-prescribing practices and carriage of penicillin-resistant Streptococcus pneumoniae: A controlled intervention trial in rural Alaska. Clinical Infectious Diseases, 34, 1543-1550.

Hennessy, T. W., Singleton, R. J., Bulkow, L. R., Bruden, D. L., Hurlburt, D. A., Parks, D., et al. (2005). Impact of heptavalent pneumococcal conjugate vaccine on invasive disease, antimicrobial resistance and colonization in Alaska Natives: Progress towards elimination of a health disparity. Vaccine, 23, 5664-5473.

Hierholzer, W. J., Jr. (1996). Principles of infectious disease epidemiology. In C. G. Mayhall (Ed.), Hospital Epidemiology and Infection Control (pp. 1-10).

Baltimore, MD: Williams \& Wilkins.

Hirschfeld, A. F., Bettinger, J. A., Victor, R. E., Davidson, D. J., Currie, A. J., Ansermino, J. M., et al. (2007). Prevalence of toll-like receptor signaling defects in apparently healthy children who developed invasive pneumococcal disease. Clinical Immunology, 122, 271-278.

Hjuler, T., Poulsen, G., Wohlfahrt, J., Kaltoft, M., Biggar, R. J., \& Melbye, M. (2008). Genetic susceptibility to severe infection in families with invasive pneumococcal disease. American Journal of Epidemiology, 167, 814-819.

Howard, C. P. (1921). Treatment of pneumonia with special reference to the use of serum. Canadian Medical Association Journal, 11, 709-713.

Hospital Care Statistics Branch. (2007). National Hospital Discharge Survey, 2005, Public Use Data File Documentation. Retrieved August 5, 2008, from Centers for Disease Control and Prevention Web Site: $\mathrm{ftp}: / / \mathrm{ftp} . c d c . g o v /$ pub/Health_Statistics/NCHS/Dataset_Documentation/NHDS/NH DS_2005_Documentation.pdf

Hsiao, Y.-S., Parker, D., Ratner, A. J., Prince, A., \& Tong, L. (2009). Crystal structures of respiratory pathogen neuraminidases. Biochemical and Biophysical Research Communications, 380, 467-471.

Hsieh, Y.-C., Wang, J.-T., Lee, W.-S., Hsueh, P.-R., Shao, P.-L., Chang, L.-Y., et al. (2006). Serotype competence and penicillin resistance in Streptococcus pneumoniae. Emerging Infectious Diseases, 12, 1709-1714.

Huang, S. S., Finkelstein, J. A., \& Lipstich, M. (2005). Modeling community- and individual-level effects of child-care attendance on pneumococcal disease. Clinical Infectious Diseases, 40, 1215-1222. 
Ibrahim, Y. M., Kerr, A. R., Silva, N. A., \& Mitchell, T. J. (2005). Contribution of the ATP-dependent protease ClpCP to the autolysis and virulence of Streptococcus pneumoniae. Infection and Immunity, 73, 730-740.

Isaacman, D. J., Strutton, D. R., Kalpas, E. A., Horowicz-Mehler, N., Stern, L. S., Casciano, R., et al. (2008). The impact of indirect (herd) protection on the costeffectiveness of pneumococcal conjugate vaccine. Clinical Therapeutics, 30, 341357.

Ishizuka, S., Yamaya, M., Suzuki, T., Takahashi, H., Ida, S., Sasaki, T., et al. (2003). Effects of rhinovirus infection on the adherence of Streptococcus pneumoniae to cultured human airway epithelial cells. Journal of Infectious Diseases, 188, 19281939.

Jackson, L. A., \& Janoff, E. N. (2008). Pneumococcal vaccination of elderly adults: New paradigms for protection. Clinical Infectious Diseases, 47, 1328-1338.

Jackson, L. A., Neuzil, K. M., Yu, O., Benson, P., Barlow, W. E., Adams, A. L., et al. (2003). Effectiveness of pneumococcal polysaccharide vaccine in older adults. New England Journal of Medicine, 348, 1747-1755.

Jansen, A. G. S. C., Sanders, E. A. M., Van Der Ende, A., Van Loon, A. M., Hoes, A. W., \& Hak, E. (2008). Invasive pneumococcal and meningococcal disease: Association with influenza virus and respiratory syncytial virus activity. Epidemiology and Infection, 136, 1448-1454.

Jansen, V. A. A., Stollenwerk, N., Jensen, H. J., Ramsay, M. E., Edmunds, W. J., \& Rhodes, C. J. (2003). Measles outbreaks in a population with declining vaccine uptake. Science, $301,804$.

Jedrzejas, M. J. (2001). Pneumococcal virulence factors: Structure and function. Microbiology and Molecular Biology Reviews, 65, 187-207.

Jefferies, J. M. C., Smith, A., Clark, S. C., Dowson, C., \& Mitchell, T. J. (2004). Genetic analysis of diverse disease-causing pneumococci indicates high levels of diversity within serotypes and capsule switching. Journal of Clinical Microbiology, 42, 5681-5688.

Jenkins, S., Brown, S., \& Farrell, D. (2008). Trends in antibacterial resistance among Streptococcus pneumoniae isolated in the USA: update from PROTEKT US Years 1-4. Annals of Clinical Microbiology and Antimicrobials, 7(1), 1.

Jose, M. V., \& Bishop, R. F. (2003). Scaling properties and symmetrical patterns in the epidemiology of rotavirus infection. Philosophical Transactions of the Royal Society of London, 358, 1625-1641. 
Jordan, E. O. (1918). The pneumococcus (Diplococcus pneumoniae). In A Text-Book of General Bacteriology (pp. 208-222). Philadelphia: W. B. Saunders.

Juhn, Y. J., Kita, H., Yawn, B. P., Boyce, T. G., Yoo, K. H., McGree, M. E., et al. (2008). Increase risk of serious pneumococcal disease in patients with asthma. Journal of Allergy and Clinical Immunology, 122, 719-723.

Kachigan, S. K. (1982). Probability topics. In Multivariate Statistical Analysis: A Conceptual Introduction (2nd ed., pp. 56-115). New York: Radius Press.

Kamboj, K. K., Kirchner, H. L., Kimmel, R., Greenspan, N. S., \& Schreiber, J. R. (2003). Significant variation in serotype-specific immunogenicity of the seven-valent Streptococcus pneumoniae capsular posysaccharide-CRM197 conjugate vaccine occurs despite vigorous $\mathrm{T}$ cell help induced by the carrier protein. Journal of Infectious Diseases, 187, 1629-1638.

Kamerling, J. P. (2000). Pneumococcal polysaccharides: A chemical view. In A. Tomasz (Ed.), Streptococcus pneumoniae: Molecular Biology and Mechanisms of Disease (pp. 81-114). Larchmont, NY: Mary Ann Liebert.

Kamo, M., \& Sasaki, A. (2005). Evolution toward multi-year periodicity in epidemics. Ecology Letters, 8, 378-385.

Keeling, M. J., \& Rohani, P. (2008a). Introduction. In Modeling Infectious Diseases in Humans and Animals (pp. 1-14). Princeton, NJ: Princeton University Press.

Keeling, M. J., \& Rohani, P. (2008b). Introduction to simple epidemic models. In Modeling Infectious Diseases in Humans and Animals (pp. 15-53). Princeton, NJ: Princeton University Press.

Keeling, M. J., \& Rohani, P. (2008c). Temporally forced models. In Modeling Infectious Diseases in Humans and Animals (pp. 155-189). Princeton, NJ: Princeton University Press.

Keeling, M. J., \& Rohani, P. (2008d). Stochastic Dynamics. In Modeling Infectious Diseases in Humans and Animals (pp. 190-230). Princeton, NJ: Princeton University Press.

Kemeny, J. J., \& Snell, J. L. (1960). Basic concepts of Markov chains. In Finite Markov Chains (pp. 24-38). Princeton, NJ: D. Van Nostrand.

Kharat, A. S., \& Tomasz, A. (2006). Drastic reduction in the virulence of Streptococcus pneumoniae expressing type 2 capsular polysaccharide but lacking choline residues in the cell wall. Molecular Microbiology, 60, 93-107. 
Kim, P. E., Musher, D. M., Glezen, W. P., Rodriguez-Barradas, M. C., Nahm, W. K., \& Wright, C. E. (1996). Association of invasive pneumococcal disease with season, atmospheric conditions, air pollution, and the isolation of respiratory viruses. Clinical Infectious Diseases, 22, 100-106.

Klein, J. O. (1981). The epidemiology of pneumococcal disease in infants and children. Reviews of Infectious Diseases, 3, 246-253.

Klemets, P., Lyytikainen, O., Ruutu, P., Ollgren, J., \& Nuorti, J. P. (2008). Invasive pneumococcal infections among persons with and without underlying medical conditions: Implications for prevention strategies. BMC Infectious Diseases, 8, 19.

Kloss, L. (2005, July). The promise of ICD-10-CM. Health Management Technology, 4849.

Klugman, K. P. (2003). The role of clonality in the global spread of fluoroquinoloneresistant bacteria. Clinical Infectious Diseases, 36, 783-785.

Koelle, K., \& Pascual, M. (2004). Disentangling extrinsic from intrinsic factors in disease dynamics: A nonlinear time series approach with an application to cholera. American Naturalist, 163, 901-913.

Komalapriya, C., Thiel, M., Romanno, M. C., Marwan, N., Schwarz, U., \& Kurths, J. (2008). Reconstruction of a system's dynamics from short trajectories. Physical Review E, 78, 1-11.

Kongstvedt, P. R. (2003). Glossary of Terms and Acronyms. In P. Kongstvedt (Ed.), Essentials of Managed Care (4th ed., p. 842). Boston: Jones and Bartlett Publishers.

Kozak, L. J., Defrances, C. J., \& Hall, M. J. (2006). National Hospital Discharge Survey: 2004 annual summary with detailed diagnosis and procedure data. Vital Health Statistics, 13(162), 193.

Ku, C.-L., Picard, C., Erdos, M., Jeurissen, A., Bustamante, J., Puel, A., et al. (2006). IRAK4 and NEMO mutations in otherwise healthy children with recurrent infections. Journal of Medical Genetics, 44, 16-23.

Kyaw, M. H., Clarke, S., Edwards, G. F., Jones, I. G., \& Campell, H. (2000). Serotypes/groups distribution and antimicrobial resistance of invasive pneumococcal isolates: Implications for vaccine strategies. Epidemiology and Infections, 125, 561-572. 
Kyaw, M. H., Rose, C. E., Fry, A. M., Singleton, J. A., Moore, Z., Zell, E. R., et al. (2005). The influence of chronic illness on the incidence of invasive pneumococcal disease in adults. Journal of Infectious Diseases, 192, 377-386.

Lande, R., Engen, S., \& Saether, B. (2002). Estimating density dependence in time-series of age-structured populations. Philosophical Transactions of the Royal Society of London, 357, 1179-1184.

Lanie, J. A., Wai-Leung, N., Kazmeirczak, K. M., Andrzejewski, T. M., Davidsen, T. M., Wayne, K. J., et al. (2007). Genome sequence of Avery's virulent serotype 2 strain D39 of Streptococcus pneumoniae and comparison with unencapsulated laboratory strain R6. Journal of Bacteriology, 189, 38-51.

Lawrenson, J. B., Klugman, K. P., Eidelman, J. I., Wasas, A., Miller, S. D., \& Lipman, J. (1988). Fatal infection caused by a multiply resistant Type 3 pneumococcus. Journal of Clinical Microbiology, 26, 1590-1591.

Lencastre, H. d., \& Tomasz, A. (2002). From ecological reservoir to disease: The nasopharynx, day care centres and drug-resistant clone of Streptococcus pneumoniae. Journal of Antimicrobial Chemotherapy, 50(Supplement S2), 75-81.

Li, S.-T., Cheng, Y.-C., \& Lin, S.-Y. (2008). A FCM-based deterministic forecasting model for fuzzy time series. Computers and Mathematics with Applications, 56, $3052-3063$

Linder, A., Hollingshead, S., Januczyk, R., Christensson, B., \& Akesson, P. (2007). Human antibody response towards the pneumococcal surface proteins PspA and PspC during invasive pneumococcal disease. Vaccine, 25, 341-345.

Lindsey, J. K. (2004a). Time series. In Statistical Analysis of Stochastic Processes in Time (pp. 213-232). West Nyak, NY: Cambridge University Press.

Lindsey, J. K. (2004b). What is a stochastic process. In Statistical Analysis of Stochastic Processes in Time (pp. 3-16). West Nyak, NY: Cambridge University Press.

Lipsitch, M., \& Moxon, E. R. (1997). Virulence and transmissibility of pathogens: What is the relationship? Trends in Microbiology, 5, 31-37.

Lipsitch, M., Whitney, C. G., Zell, E., Kaijalainen, T., Dagan, R., \& Malley, R. (2005). Are anticapsular antibodies the primary mechanism of protection against invasive pneumococcal disease? PLoS Medicine, 2, 0062-0068.

Lloyd, A. L. (2001). Realistic distributions of infectious periods in epidemic models: Changing patterns of persistence and dynamics. Theoretical Population Biology, $60,59-71$. 
Lloyd, A. L. (2004). Estimating variability in models for recurrent epidemics: Assessing the use of moment closure techniques. Theoretical Population Biology, 65, 49-65.

Lofgren, E., Fefferman, N. H., Naumov, Y. N., Gorski, J., \& Naumova, E. N. (2007). Influenza seasonality: Underlying causes and modeling theories. Journal of Virology, 81, 5429-5436.

Lopez, R. (2006). Pneumococcus: The sugar-coated bacteria. International Microbiology, 9, 179-190.

Lou, Y., \& Zack, M. (2005). Computations of confidence intervals for estimates in the United States National Hospital Discharge Survey, 1979-2000. Preventing Chronic Disease, 2, 1- 12.

Machlin, S., Cohen, J., \& Thorpe, J. (2000). Measuring inpatient care use in the United States: A comparison across five federal data sources. Journal of Economic and Social Measurement, 26, 141-51.

MacLeod, C. M., \& Avery, O. T. (1941). The occurrence during acute infections of a protein not normally present in the blood. Part III. Immunological properties of the C-reactive protein and its differentiation from normal blood proteins. Journal of Experimental Medicine, 73, 191-200.

Madhi, S. A., Whitney, C. G., \& Nohynek, H. (2008). Lessons learned from clinical trials evaluating pneumococcal conjugate vaccine efficacy against pneumonia and invasive disease. Vaccine, 26, B9-B15.

Magee, A. D., \& Yother, J. (2001). Requirement for capsule in colonization by Streptococcus pneumoniae. Infection and Immunity, 69, 3755-3761.

Maekelae, P. H., \& Kayhty, H. (2004). Vaccine-induced immunity to pneumococcal infection. In E. I. Tuomanen, T. J. Mitchell, D. A. Morrison \& B. G. Spratt (Eds.), The Pneumococcus (pp. 403-420). Washington, D.C.: ASM Press.

Maki, D. G. (2004). Pneumococcal bacteremia: Lessons learned, yet more to learn. Mayo Clinic Proceedings, 79, 599-603.

McBean, A. M., Park, Y.-T., Caldwell, D., \& Yu, X. (2005). Declining invasive pneumococcal disease in the U.S. elderly. Vaccine, 23, 5641-5645.

McDaniel, L. S., \& Swialto, E. (2004). Pneumococcal disease: Pathogenesis, treatment, and prevention. Infectious Clinical Disease Practice, 12, 93-98. 
McEllistrem, M. C., Mendelssohn, A. B., Pass, M. A., Elliot, J. A., Whitney, C. G., Kolano, J. A., et al. (2002). Recurrent invasive pneumococcal disease in individuals with Human Immunodeficiency Virus infection. Journal of Infectious Diseases, 185, 1364-1368.

McGee, L. (2007). The coming of age of niche vaccines? Effect of vaccines on resistance profiles in Streptococcus pneumoniae. Current Opinion in Microbiology, 10, 473478.

McGee, L., McDougal, L., Zhou, J., Spratt, B. G., Tenover, F. C., George, R., et al. (2001). Nomenclature of major antimicrobial-resistant clones of Streptococcus pneumoniae defined by the Pneumococcal Molecular Epidemiology Network. Journal of Clinical Microbiology, 39, 2565-2571.

McLemore, T., \& Pokras, R. (2001). Data quality in the National Hospital Discharge Survey. Paper presented at the meeting of the Statistics Canada Symposium 2001. Ottawa, Canada.

McNabb, S. J., Jajoksy, R. A., Hall-Baker, P. A., Adams, D. A., Sharp, P., Anderson, W. J., et al. (2007). Summary of Notifiable Diseases-United States, 2005. Morbidity and Mortality Weekly Report, 54, 32-35.

Metlay, J. P., Fishman, N. O., Joffe, M. N., Kallan, M. J., Chittams, J. L., \& Edelstein, P. H. (2006). Macrolide resistance in adults with bacteremic pneumococcal pneumonia. Emerging Infectious Diseases, 12, 1223-1230.

Meyn, S. P., \& Tweedie, R. L. (1993). Ergodicity. In Markov Chains and Stochastic Stability (pp. 313-333). London: Springer-Verlag.

Miernyk, K. M., Parkinson, A. J., Rudolph, K. M., Peterson, K. M., Bulkow, L. R., Greenberg, D. P., et al. (2000). Immunogenicity of a heptavalent pneumococcal conjugate vaccine in Apache and Navaho Indian, Alaska Native, and non-native American children aged $<2$ years. Clinical Infectious Diseases, 31, 34-41.

Millar, E. V., Watt, J. P., Bronsdon, M. A., Dallas, J., Reid, R., Santosham, M., et al. (2008). Indirect effect of 7-valent pneumococcal conjugate vaccine on pneumococcal colonization among unvaccinated household members. Clinical Infectious Diseases, 47, 989-996.

Millar, E. V., O'Brien, K. L., Watt, J. P., Bronsdon, M. A., Dallas, J., Whitney, C. G., et al. (2006). Effect of community-wide conjugate pneumococcal vaccine use in infancy on nasopharyngeal carriage through 3 years of age: A cross-sectional study in a high-risk population. Clinical Infectious Diseases, 43, 8-15. 
Mollison, D., Isham, V., \& Grenfell, B. (1994). Epidemics: Models and data. Journal of the Royal Statistical Society. Series A (Statistics in Society), 157, 115-149.

Moore, M. R., Gertz, R. E., Woodbury, R. L., Barkocy-Gallagher, G. A., Schaffner, W., Lexau, C., et al. (2008). Population snapshot of emergent Streptococcus pneumoniae serotype 19A in the United States, 2005. Journal of Infectious Diseases, 197, 1016-1027.

Moore, L. J., Gilbey, A. M., Dowson, C. G., Pridmore, A. C., Dower, S. K., \& Read, R. C. (2007). Proinflammatory activation of Toll-like receptor-2 during exposure of penicillin-resistant Streptococcus pneumoniae to beta-lactam antibiotics. Journal of Antimicrobial Chemotherapy, 59, 35-42.

Moore, M. R., Hyde, T. B., Hennessy, T. W., Parks, D. J., Reasonover, A. L., HarkerJones, M., et al. (2004). Impact of a conjugate vaccine on community-wide carriage of nonsusceptable Streptococcus pneumoniae in Alaska. Journal of Infectious Diseases, 190, 2031-2038.

Moscoso, M., Garcia, E., \& Lopez, R. (2006). Biofilm formation by Streptococcus pneumoniae: Role of choline, extracellular DNA, and capsular polysaccharide in microbial accretion. Journal of Bacteriology, 188, 785-7795.

Movig, K. L. L., Leufkens, H. G. M., Lenderink, A. W., \& Egberts, A. C. G. (2003). Validity of hospital discharge International Classification of Diseases (ICD) codes for identifying patients with hyponatremia. Journal of Clinical Epidemiology, 56, 530-535.

Mufson, M. A. (2000). Antibody response of pneumococcal vaccine: need for booster dosing? Antimicrobial Agents, 14, 107-112.

Müller-Graf, C. D. M., Whatmore, A. M., King, S. J., Trzcinski, K., Pickerill, A. P., Doherty, N., et al. (1999). Population biology of Streptococcus pneumoniae isolated from oropharyngeal carriage and invasive disease. Microbiology, 145, 3283-3293.

Myers, C., \& Gervaix, A. (2007). Streptococcus pneumoniae bacteraiemia in children. International Journal of Antimicrobial Agents, 30(S1), 24-28.

National Center for Health Statistics. (2007a). National Hospital Discharge Survey description. Retrieved August 5, 2008, from Centers for Disease Control and Prevention Web Site: http://www.cdc.gov/nchs/about/major/hdasd/nhdsdes.htm

National Center for Health Statistics. (2007b). National Hospital Discharge Survey sample design. Retrieved August 5, 2008, from Centers for Disease Control and Prevention Web Site: http://www.cdc.gov/nchs/about/major/hdasd/nhdssam.htm 
National Center for Health Statistics. (2007c). Nhds05readme.txt. Retrieved August 5, 2008, from Centers for Disease Control and Prevention Web Site:

ftp://ftp.cdc.gov/pub/Health_Statistics/NCHS/Dataset_Documentation/NHDS/nhd s05readme.txt

Nelson, K. E. (2007). Epidemiology of infectious disease: General Principles. In K. E. Nelson \& C. M. Williams (Eds.), Infectious Disease Epidemiology-Theory and Practice (2nd ed., pp. 25-62). Boston: Jones and Bartlett.

Nelson, A. L., Roche, A. M., Gould, J. M., Chim, K., Ratner, A. J., \& Weiser, J. N. (2007). Capsule enhances pneumococcal colonization by limiting mucusmediated clearance. Infection and Immunity, 75, 83-90.

Noah, N. D. (1989). Cyclical patterns and predictability in infection. Epidemiology and Infection, 102, 175-190.

Normark, B. H., Kalin, M., Ortqvist, A., Akerlund, T., Liljequist, B. O., Hedlund, J., et al. (2001). Dynamics of penicillin-susceptible clones in invasive pneumococcal disease. Journal of Infectious Diseases, 184, 861-869.

Nuermberger, E. L., \& Bishai, W. R. (2004). Antibiotic resistance in Streptococcus pneumoniae: What does the future hold. Clinical Infectious Diseases, 38, S363S371.

Nuorti, J. P., Butler, J. C., Farley, M. M., Harrison, L. H., McGeer, A., Kolczak, M. S., et al. (2000). Cigarette smoking and Invasive Pnuemococcal Disease. New England Journal of Medicine, 342, 681-689.

Nuorti, J. P., Butler, J. C., Gelling, L., Kool, J. L., Reingold, A. L., \& Vugia, D. J. (2000). Epidemiologic relation between HIV and invasive pneumococcal disease in San Francisco County, California. Annals of Internal Medicine, 132, 182-190.

O'Brien, K. L., Millar, E. V., Zell, E. R., Bronsdon, M., Weatherholtz, R., Reid, R., et al. (2007). Effect of pneumococcal conjugate vaccine on nasopharyngeal colonization among immunized and unimmunized children in a communityrandomized trial. Journal of Infectious Diseases, 196, 1211-1220.

Obert, C., Sublett, J., Kaushal, D., Hinojosa, E., Barton, T., Tuomanen, E., et al. (2006). Identification of a candidate Streptococcus pneumoniae core genome and regions of diversity correlated with invasive pneumococcal disease. Infection and Immunity, 74, 4766-4777.

Obert, C. A., Gao, G., Sublett, J., Tuomanen, E. I., \& Orihuela, C. J. (2007). Assessment of molecular typing methods to determine invasiveness and to differentiate clones of Streptococcus pneumoniae. Infection, Genetics and Evolution, 7, 708-716. 
Ohlsen, K., Hacker, J., \& Ziebuhr, W. (2004). Genome variability in gram-positive pathogenic bacteria-Impact on virulence and evolution. Current Genomics, 5, 589-600.

Olsen, L. F., \& Schaffer, W. M. (1990). Chaos versus noisy periodicity: Alternative hypotheses for childhood epidemics. Science, 249, 499-504.

Oregon Department of Human Services (2004). 2004 State of Oregon Selected Communicable Disease Summary. Salem, OR: State of Oregon.

Orihuela, C. J., Radin, J. N., Sublett, J. E., Gao, G., Kaushal, D., \& Tuomanen, E. I. (2004). Microarray analysis of pneumococcal gene expression during invasive disease. Infection and Immunity, 72, 5582-5596.

Orihuela, C. J., \& Tuomanen, E. (2006). Streptococcus pneumoniae: Invasion and Inflammation. In V. Fischetti, R. Novick, J. Ferretti, D. Portnoy \& J. Rood (Eds.), Gram-Positive Pathogens. Washington, DC: ASM Press.

Ortqvist, A., Hedlund, J., Grillner, L., Jalonen, E., Kallings, I., Leinonen, M., et al. (1990). Aetiology, outcome and prognostic factors in community-acquired pneumonia requiring hospitalization. European Respiratory Journal, 3(10), 1105 1113.

Osler, W. (1899). Lobar pneumonia. In The Principles and Practice of Medicine (pp. 108-137). New York: Appleton and Company.

Overturf, G. D. (2000). Technical report: Prevention of pneumococcal infections, including the use of pneumococcal conjugate and polysaccharide vaccines and antibiotic prophylaxis. Pediatrics, 106, 367-376.

Pai, R., Moore, M. R., Pilishvili, T., Gertz, R. E., Whitney, C. G., \& Beall, B. (2005). Postvaccine genetic structure of Streptococcus pneumoniae serotype 19A from children in the United States. Journal of Infectious Diseases, 192, 1988-1995.

Papoulis, A. (1984). Markov processes. In Probability, Random Variables and Stochastic Processes (2nd ed., pp. 538). Columbus, OH: McGraw-Hill.

Parker, J. D., Schenker, N., Ingram, D. D., Weed, J. A., Heck, K. E., \& Madans, J. H. (2004). Bridging between two standards for collecting information on race and ethnicity: An application to Census 2000 and vital rates. Public Health Reports, $119,192-205$

Parker, R. H., Davison, R., Paterson, P. Y., \& Madden, G. M. (1970). Erithromycinresistant pneumococcal osteomyelitis. American Journal of Medicine, 48, 132136. 
Parsons, H. K., \& Dockrell, D. H. (2002). The burden of invasive pneumococcal disease and the potential for reduction by immunization. International Journal of Antimicrobial Agents, 19, 85-93.

Pasteur, L. (1881). Note sur la maladie nouvelle provoquee par la salive d'un enfant mort de la rage. Bulletin de l'Academie de Medicine (Paris) Series 2, 10, 94-103.

Pelton, S. I., Huot, H., Finkelstein, J. A., Bishop, C. J., Hsu, K. K., Kellenberg, J., et al. (2007). Emergence of 19A as virulent and multidrug resistant pneumococcus in Massachusetts following universal immunization of infants with pneumococcal conjugate vaccine. Pediatric Infectious Disease Journal, 26, 468-472.

Penaranda, M., Falco, V., Payeras, A., Jordano, Q., Curran, A., Pareja, A., et al. (2007). Effectiveness of polysaccharide pneumococcal vaccine in HIV-infected patients: A case-control study. Clinical Infectious Diseases, 45, e82-e87.

Penn, R. L., \& Betts, R. F. (2003). Lower Respiratory Tract Infections (Including Tuberculosis. In R. Betts, S. Chapman \& R. Penn (Eds.), Reese and Betts' A Practical Approach to Infectious Diseases (5th ed., pp. 295-371). Philadelphia: Lippincott Williams \& Wilkins.

Pericone, C. D., Overweg, K., Hermans, P. W. M., \& Weiser, J. N. (2000). Inhibitory and bactericidal effects of hydrogen peroxide production by Streptococcus pneumoniae on other Inhabitants of the upper respiratory tract. Infection and Immunity, 68(7), 3990-3997.

Pericone, C. D., Park, S., Imlay, J. A., \& Weiser, J. N. (2003). Factors contributing to hydrogen peroxide resistance in Streptococcus pneumoniae include pyruvate oxidase $(\mathrm{SpxB})$ and avoidance of the toxic effects of the Fenton reaction. Journal of Bacteriology, 185, 6815-6825.

Petermann, T., \& Rios, P. D. L. (2004). Cluster approximations for epidemic processes: A systematic description of correlations beyond the pair level. Journal of Theoretical Biology, 229, 1-11.

Picard, C., Puel, A., Bonnet, M., Ku, C.-L., Bustamante, J., Yang, K., et al. (2003). Pyogenic bacterial infections in humans with IRAK-4 deficiency. Science, 299, 2076-2079.

Pletz, M. W., Maus, U., Krug, N., Welte, T., \& Lode, H. (2008). Pneumococcal vaccines: mechanism of action, impact on epidemiology and adaptation of the species. Antimicrobial Agents, 32, 199-206. 
Poehling, K. A., Talbot, T. R., Griffin, M. R., Craig, A. S., Whitney, C. G., Zell, E., et al. (2006). Invasive pneumococcal disease among infants before and after introduction of pneumococcal conjugate vaccine. Journal of the American Medical Association, 295, 1668-1674.

Poland, G. A. (1999). The burden of pneumococcal disease: The role of conjugate vaccines. Vaccine, 17, 1764-1679.

Popovic, J. R. (2001). National Hospital Discharge Survey: Annual summary with detailed diagnosis and procedure data. Vital Health Statistics, 13, 1-206.

Rajam, G., Philips, D. J., White, E., Anderton, J., Hooper, C. W., Sampson, J. S., et al. (2008). A functional epitope of the pneumococcal surface adhesin A activates nasopharyngeal cells and increases bacterial internalization. Microbial Pathogenesis, 44, 186-196.

Randolph, D. A., \& Lewis, D. B. (2006). Transient deficiencies of T-cell-mediated immunity in the neonate. In A. Pollard \& A. Finn (Eds.), Hot Topics in Infection and Immunity in Children III. New York: Springer.

Regev-Yochay, G., Lipsitch, M., Basset, A., Rubinstein, E., Dagan, R., Raz, M., et al. (2009). The pneumococcal pilus predicts the absence of Staphylococcus aureus co-colonization in pneumococcal carriers. Clinical Infectious Diseases, 48, 760 763.

Regev-Yochay, G., Trzcinksi, K., Thompson, C. M., Malley, R., \& Lipsitch, M. (2006). Interference between Streptococcus pneumoniae and Staphylococcus aureus: In vitro hydrogen peroxide-mediated killing by Streptococcus pneumoniae. Journal of Bacteriology, 188, 4996-5001.

Resti, M., Micheli, A., Moriondo, M., Becciolini, L., Cortimiglia, M., Celementina, C., et al. (2009). Comparison of the effect of antibiotic treatment on the possibility of diagnosing Invasive Pneumococcal Disease by culture or molecular methods: A prospective, observational study of children and adolescents with proven pneumococcal infection. Clinical Therapeutics, 31, 1266-1273.

Ribes, S., Taberner, F., Cabellos, C., Tubau, F., Ardanuy, C., Gerber, J., et al. (2008). Contribution of capsular and clonal types and B-lactam resistance to the severity of experimental pneumococcal meningitis. Microbes and Infection, 10, 129-134.

Rice, L. B. (2006). Antimicrobial resistance in Gram-positive bacteria. American Journal of Medicine, 119, S11-S19. 
Richter, S. S., Heilmann, K. P., Coffman, S. L., Huynh, H. K., Brueggemann, A. B., Pfaller, M. A., et al. (2002). The molecular epidemiology of penicillin-resistant Streptococcus pneumoniae in the United States, 1994-2000. Clinical Infectious Diseases, 34, 330-339.

Rieux, V. (2002). Les facteurs de virulence de Streptococcus pneumoniae. Medicine Maladie et Infection, 32, 1-12.

Riley, L. W. (2004). Distinguishing pathovars from nonpathovars: Streptococcus pneumoniae. In Molecular Epidemiology of Infectious Disease. Washington, DC: ASM Press.

Riley, S. (2007). Large-scale spatial-transmission models of infectious disease. Science, 316(1298-1301).

Robinson, K. A., Baughman, W., Rothrock, G., Barrett, N. L., Pass, M., Lexau, C., et al. (2001). Epidemiology of invasive Streptococcus pneumoniae infections in the United States, 1995-1998: Opportunities for prevention in the conjugate vaccine era. Journal of the American Medical Association, 285, 1729-1735.

Rodriquez-Arias, M. A., \& Rodo, X. (2004). A primer of the study of transitory dynamics in ecological series using the scale-dependent correlation analysis. Oecologia, $138,485-504$.

Rohani, P., Earn, D. J. D., \& Grenfell, B. T. (1999). Opposite patterns of synchrony in sympatric disease populations. Science, 286, 968-971.

Romney, M. G., Hull, M. W., Gustafson, R., Sandhu, J., Champagne, S., Wong, T., et al. (2008). Large community outbreak of Streptococcus pneumoniae serotype 5 invasive infection in an impoverished, urban population. Clinical Infectious Diseases, 47, 768-774.

Rosen, F. S., \& Ryan, M. W. (2007). The prevalence of colonization with drug-resistant pneumococci among adult workers in children's daycare. ENT-Ear, Nose \& Throat Journal, 86, 38-44.

Rudolph, K. M., Parkinson, A. J., Reasonover, A. L., Parks, D. J., \& Butler, J. C. (2000). Serotype distribution and antimicrobial resistance patterns of invasive isolates of Streptococcus pneumoniae: Alaska, 1991-1998. Journal of Infectious Diseases, $182,490-496$.

Rytel, M. W., Dee, T. H., Ferstenfeld, J. E., \& Hensley, G. T. (1974). Possible pathogenetic role of capsular antigens in fulminant pneumococcal disease with disseminated intravascular coagulation. American Journal of Medicine, 57, 889896. 
Schoenman, J. A., Sutton, J. P., Kintala, S., Love, D., \& Maw, R. (2005). The value of hospital discharge databases. Retrieved May 21, 2007, from Agency for Healthcare Research and Quality Web Site: http://www.hcupus.ahrq.gov/reports/final_report.pdf

Schutze, G. E., Mason, E. O., Barson, W. J., Kim, K. S., Wald, E. R., Givner, L. B., et al. (2002). Invasive pneumococcal infections in children with asplenia. Pediatric Infectious Disease Journal, 21, 278-282.

Serrano, I., Melo-Cristino, J., \& Ramirez, M. (2006). Heterogeneity of pneumococcal phase variants in invasive human infections. BMC Microbiology(6), 1-6.

Shah, S. S., \& Ratner, A. J. (2005). Trends in invasive pneumococcal disease-associated hospitalizations. Clinical Infectious Diseases, 42, e1-e5.

Simell, B., Lahdenkari, M., Reunanen, A., Kaeyhty, H., \& Vaevaeinen, M. (2008). Effects of ageing and gender on naturally acquired antibodies to pneumococcal capsular polysaccharides and virulence-associated proteins. Clinical and Vaccine Immunology, 15, 1391-1397.

Singleton, R. J., Butler, J. C., Bulkow, L. R., Hurlburt, D., O'Brien, K. L., Doan, W., et al. (2007). Invasive pneumococcal disease epidemiology and effectiveness of 23valent pneumococcal polysaccharide vaccine in Alaska Native adults. Vaccine, 25(2288-2295).

Sisk, J. E., Whang, W., Butler, J. C., Sneller, V. P., \& Whitney, C. G. (2003). Costeffectiveness of vaccination against invasive pneumococcal disease among people 50 through 64 years of age: Role of comorbid conditions and race. Annals of Internal Medicine, 138, 960-968.

Sjoestroem, K., Spindler, C., Kalin, M., Sandgren, A., Kuehlman-Berenzon, S., \& Henriques-Normark, B. (2006). Clonal and capsular types decide whether pneumococci will act as a primary or opportunistic infection. Clinical Infectious Diseases, 42, 451-459.

Sleeman, K. L., Griffiths, D., Shackley, F., Diggle, L., Gupta, S., Maiden, M. C., et al. (2006). Capsular serotype-specific attack rates and duration of carriage of Streptococcus pneumoniae in a population of children. Journal of Infectious Diseases, 194, 682-688.

Smith, C. H., Erlandson, M., Shulman, I., \& Stern, G. (1957). Hazard of severe infections in splenectomized infants and children. American Journal of Medicine, 22, 390404. 
Smith, M. D., Sheppard, C. L., Hogan, A., Harrison, T. G., Dance, D. A. B., Derrington, P., et al. (2009). Diagnosis of Streptococcus pneumoniae infections in adults with bacteremia and community-acquired pneumonia: Clinical comparison of pneumococcal PCR and urinary antigen detection. Journal of Clinical Microbiology, 47, 1046-1049.

Smith, P. J., Nuorti, J. P., Singleton, J. A., Zhao, Z., \& Wolter, K. M. (2007). Effect of vaccine shortages on timeliness of pneumococcal conjugate vaccination: Results from the 2001-2005 National Immunization Survey. Pediatrics, 120, 1165-1173.

Sniadack, D. H., Schwartz, B., Lipman, H., Bogaerts, J., Butler, J. C., Dagan, R., et al. (1995). Potential interventions for the prevention of childhood pneumonia: Geographic and temporal differences in serotype and serogroup distribution of sterile site pneumococcal isolates from children-implications for vaccine strategies. Pediatric Infectious Disease Journal, 14, 503-510.

Soper, H. E. (1929). The interpretation of periodicity in disease prevalence. Journal of the Royal Statistical Society, 92, 34-73.

Sorrell, I., Pedersen, A. B., Hails, R. S., \& Boots, M. (2009). The evolution of covert, silent infection as a parasite strategy [Electronic Version]. Proceedings of the Royal Society B, 1-10. Retrieved April 7, 2009, from http://rspb.royalsocietypublishing.org/content/early/2009/03/06/rspb.2008.1915.fu $\underline{11 . h t m l}$

SPSS. (2008). SPSS Statistics 17.0 Algorithms. Retrieved January 5, 2009, from http://support.spss.com/ProductsExt/SPSS/ESD/17/Download/User\%20Manuals/ English/SPSS\%20Statistics\%2017.0\%20Algorithms.pdf

Steenhoff, A. P., Wood, S. M., Rutstein, R. M., Wahl, A., McGowan, K. L., \& Shah, S. S. (2008). Invasive pneumococcal disease among Human Immunodeficiency Virus-infected children, 1989-2006. Pediatric Infectious Disease Journal, 27, 886-891.

Stegemann, S., Dahlberg, S., Kroeger, A., Gereke, M., Bruder, D., Henriques-Normark, B., et al. (2009). Increased susceptibility for superinfection with Streptococcus pneumoniae during influenza virus infection is not caused by TLR7-mediated lymphopenia. PLOS ONE, 4, 1-9.

Sternberg, G. M. (1881). A fatal form of septicemia in the rabbit, produced by the subcutaneous injection of human saliva. Annual Reports of the National Board of Health, 3, 87-108. 
Stevens, D. L. (2000). Group A Beta-Hemolytic Streptococci: Virulence Factors, Pathogenesis, and Spectrum of Clinical Infections. In D. Stevens \& E. Kaplan (Eds.), Streptococcal Infections: Clinical Aspects, Microbiology, and Molecular Pathogenesis (pp. 19-38). New York: Oxford University Press.

Stewart, I. (2002). Chaos from order. In Does God Play Dice? The New Mathematics of Chaos (2nd ed., pp. 12). Malden, MA: Blackwell.

Stone, L., Olinky, R., \& Huppert, A. (2007). Seasonal dynamics of recurrent epidemics. Nature, 446, 533-536.

Sutton, K. L., Banks, H. T., \& Castilo-Chavez, C. (2008). Estimation of invasive pneumococcal disease dynamics parameters and the impact of conjugate vaccination in Australia. Mathematical Biosciences and Engineering, 5, 175-204.

Talbot, T. R., Poehling, K. A., Hartert, T. V., Arbogast, P. G., Halasa, N. B., Edwards, K. M., et al. (2005). Seasonality of invasive pneumococcal disease: Temporal relation to documented influenza and respiratory syncytial viral circulation. American Journal of Medicine, 118, 285-291.

Taniguchi, M., \& Kakizawa, Y. (2000). Asymptotic theory of estimation and testing for stochastic processes. In Asymptotic Theory of Statistical Inference for Time Series (pp. 51-165). New York: Springer-Verlag.

Tasslimi, A., Wenger, P., Pentakota, S. R., Brimacombe, M., Burday, M., \& Sinha, A. (2008). Invasive pneumococcal disease in an underimmunized, high HIV prevalence population. Journal of Infection, 56, 99-102.

Taylor, S. N., \& Sanders, C. V. (1999). Unusual manifestations of invasive pneumococcal disease. American Journal of Medicine, 107, 12S-27S.

Temime, L., Boelle, P.-Y., Opatowski, L., \& Guillemot, D. (2008). Impact of capsular switch on invasive pneumococcal disease incidence in a vaccinated population. PLOS ONE, 3, 1-7.

Tettelin, H., Nelson, K. E., Paulson, I. T., Eisen, J. A., Read, T. D., Peterson, S., et al. (2001). Complete genome sequence of a virulent isolate of Streptococcus pneumoniae. Science, 293, 498-506.

Tomasz, A. (1999). New faces of an old pathogen: Emergence and spread of multidrugresistant Streptococcus pneumoniae. American Journal of Medicine, 107, 55S$62 \mathrm{~S}$. 
Tong, H. H., Weiser, J. N., James, M. A., \& DeMaria, T. F. (2001). Effect of Influenza A virus infection on nasopharyngeal colonization and Otitis Media induced by transparent or opaque phenotype variants of Streptococcus pneumoniae in the Chinchilla model. Infection and Immunity, 69, 602-606.

Torres, J., \& Bisno, A. L. (1973). Hyposplenism and pneumococcemia. American Journal of Medicine, 55, 851-855.

Trochim, W. M. K. (2001). Sampling. In The Research Methods Knowledge Base (2nd ed., pp. 41-59). Mason, OH: Thompson.

Trzcinksi, K., Thompson, C. M., \& Lipsitch, M. (2004). Single-step capsular transformation and acquisition of penicillin resistance in Streptococcus pneumoniae. Journal of Bacteriology, 186, 3447-3452.

Tsai, C. J., Griffin, M. R., Nuorti, J. P., \& Grijalva, C. G. (2008). Changing epidemiology of pneumococcal meningitis after the introduction of pneumococcal conjugate vaccine in the United States. Clinical Infectious Diseases, 46, 1664-1672.

Tunkel, A. R., \& Scheld, W. M. (2003). Central Nervous System infections. In R. Betts, S. Chapman \& R. Penn (Eds.), A Practical Approach to Infectious Diseases (pp. 173-221). Philadelphia: Lippincott Williams \& Wilkins.

Turett, G. S., Blum, S., \& Telzak, E. E. (2001). Recurrent pneumococcal bacteremia. Archives of Internal Medicine, 161, 2141-2144.

van de Garde, E. M. W., Ooosterheert, J. J., Bonten, M., Kaplan, R. C., \& Leufkens, H. G. M. (2007). International Classification of Diseases codes showed modest sensitivity for detecting community-acquired pnemonia. Journal of Clinical Epidemiology, 60, 834-838.

Varon, E. (2001). Infections graves a pneumocoques: Facteurs de pathogenicite. Archives Pediatrics, 8, 752-756.

Verdasca, J., Gama, M. M. T. d., Nunes, A., Bernardino, N. R., Pacheco, J. M., \& Gomes, M. C. (2005). Recurrent epidemics in small world networks. Journal of Theoretical Biology, 233, 553-561.

Verhelst, R., Kaiijalainen, T., Baere, T. D., Verschraegen, G., Claeys, G., Simaey, L. V., et al. (2003). Comparison of five genotyping techniques for identification of optochin-resistant pneumococcus-like isolates. Journal of Clinical Microbiology, 41, 3521-3525. 
Viboud, C., Bjornstad, O. N., Smith, D. L., Simonsen, L., Miller, M. A., \& Grenfell, B. T. (2006). Synchrony, waves, and spatial hierarchies in the spread of influenza. Science, 312, 447-451.

Viboud, C., Boelle, P., Carrat, F., Valleron, A., \& Flahault, A. (2003). Prediction of the spread of influenza epidemics by the method of analogues. American Journal of Epidemiology, 158, 996-1006.

Wallinga, J., Edmunds, W. J., \& Kretzschmar, M. (1999). Perspective: Human contact patterns and the spread of airborne infectious diseases. Trends in Microbiology, 7, $372-377$.

Watson, D. A., Musher, D. M., Jacobson, J. W., \& Verhoef, J. (1993). A brief history of the pneumococcus in biomedical research: A panoply of scientific discovery. Clinical Infectious Diseases, 17, 913-924.

Wei, W. W. S. (1994a). Overview. In Time Series Analysis-Univariate and Multivariate Methods (pp. 1-5). Redwood City, CA: Addison-Wesley Publishing Company.

Wei, W. W. S. (1994b). Fundamental Concepts. In Time Series Analysis-Univariate and Multivariate Methods (pp. 6-31). Redwood City, CA: Addison-Wesley.

Wei, W. W. S. (1994c). Nonstationary time series models. In Time Series AnalysisUnivariate and Multivariate Methods (pp. 67-103). Redwood City, CA.

Wei, W. W. S. (1994d). Seasonal Time Series Models. In Time Series AnalysisUnivariate and Multivariate Methods (pp. 157-183). Redwood City, CA: Addison-Wesley Publishing.

Wei, W. W. S. (1994e). Fourier analysis. In Time Series Analysis-Univariate and Multivariate Methods (pp. 206-321). Redwood City, CA: Addison-Wesley.

Weiser, J. N., Austrian, R., Sreenivasan, P. K., \& Masure, H. R. (1994). Phase variation in pneumococcal opacity: Relationship between colonial morphology and nasopharyngeal colonization. Infection and Immunity, 62, 2582-2589.

Welch, W. H. (1892). The micrococcus lanceolatus, with a special reference to the etiology of acute lobar pneumonia. Bulletin of the Johns Hopkins Hospital, 3, 125-139.

Werno, A. M., \& Murdoch, D. R. (2008). Laboratory diagnosis of invasive pneumococcal disease. Clinical Infectious Diseases, 46, 926-932. 
Whatmore, A. M., King, S. J., Doherty, N. C., Sturgeon, D., Chanter, N., \& Dowson, C. G. (1999). Molecular characterization of equine isolates of Streptococcus pneumoniae: Natural disruption of genes encoding the virulence factors pneumolysin and autolysin. Infection and Immunity, 67, 2776-2782.

Whitney, C. G., Farley, M. M., Hadler, J., Harrison, L. H., Lexau, C., Reingold, A., et al. (2000). Increasing prevalence of multidrug-resistant Streptococcus pneumoniae in the United States. New England Journal of Medicine, 343, 1917-1924.

Whitney, C. G., Pilishvili, T., Farley, M. M., Schaffner, W., Craig, A. S., Lynfield, R., et al. (2006). Effectiveness of seven-valent pneumococcal conjugate vaccine against invasive pneumococcal disease: A matched case-control study. Lancet, 368, 14951502.

Whitney, C. G., Schaffner, W., \& Butler, J. C. (2001). Rethinking recommendations for use of pneumococcal vaccines in adults. Clinical Infectious Diseases, 33, 662675.

Wilkinson, M., Bulloch, B., \& Smith, M. (2009). Prevalence of occult bacteremia in children aged 3 to 36 months presenting to the emergency department with fever in the postpneumococcal conjugate vaccine era. Academic Emergency Medicine, $16,220-225$.

Williams, C., \& Masterton, R. (2008). Pneumococcal vaccination in the 21 st century. Journal of Infection, 56, 13-19.

Wilson, E. B., \& Worcester, J. (1945). The law of mass action in epidemiology. Proceedings of the National Academy of Sciences of the United States of America, 31, 24-34The Law of Mass Action in Epidemiology.

Woude, M. W. v. d., \& Baumler, A. J. (2004). Phase and antigenic variation in bacteria. Clinical Microbiology Reviews, 17, 581-611.

Xia, Y., Bjornstad, O. N., \& Grenfell, B. T. (2004). Measles metapopulation dynamics: A gravity model for epidemiological coupling and dynamics. American Naturalist, $164,267-281$.

Yesilkaya, H., Kadioglu, A., Gingles, N., Alexander, J. E., Mitchell, T. J., \& Andrew, P. W. (2000). Role of manganese-containing superoxide dismutase in oxidative stress and virulence of Streptococcus pneumoniae. Infection and Immunity, 68, 2819-2826. 
Yulmetyev, R., Emelyanova, N., Demin, S., Gafarov, F., Haenggii, P., \& Yumetyeva, D. (2003). Fluctuations and noise in stochastic spread of respiratory infection epidemics in social networks. American Institute of Physics Conference Proceedings, 655, 408-417.

Yulmetyev, R. M., Yulmetyeva, D., \& Gafarov, F. M. (2005). How chaosity and randomness control human health. Physica A, 354, 404-414.

Ziemer, R. E., Tranter, W. H., \& Fannin, D. R. (1983). Fourier series and the Fourier Transform. In Signals and Systems-Continuous and Discrete (pp. 86-168). New York: Macmillan. 
APPENDIX

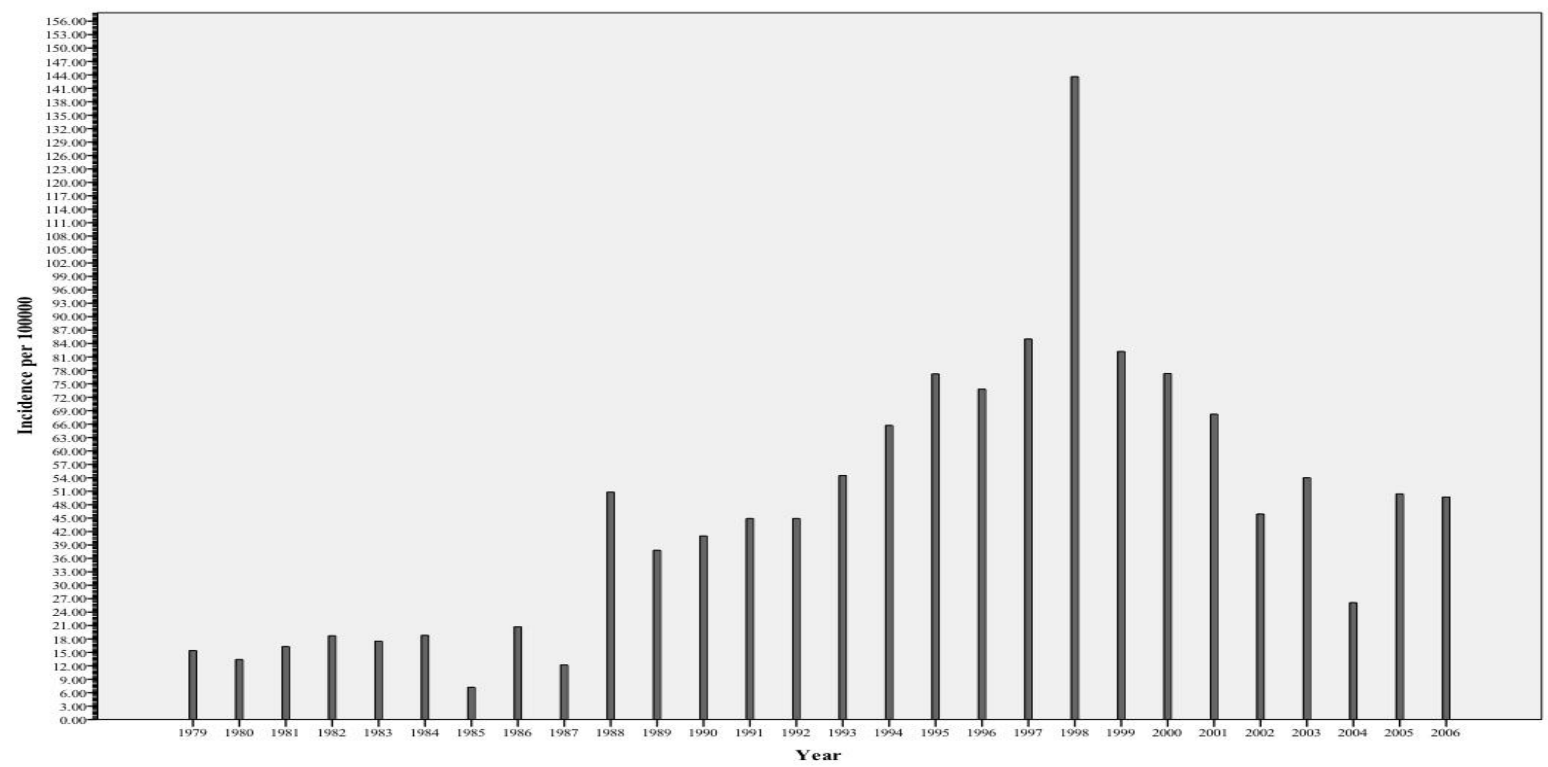

Figure 1. Incidence of IPD in the United States, 1979-2006, per 100000.

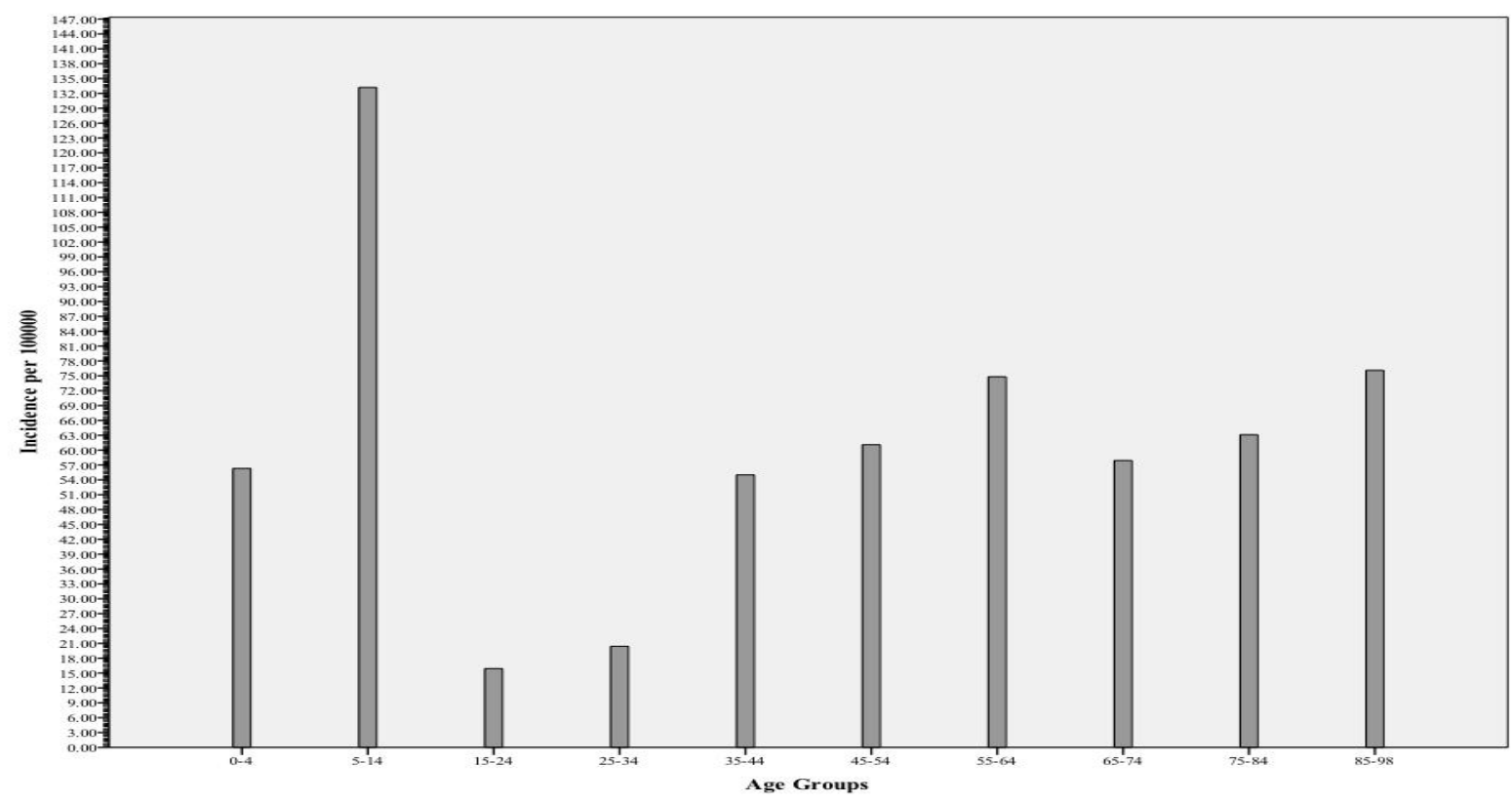

Figure 2. Incidence of IPD in the United States, 1979-2006, by age. 


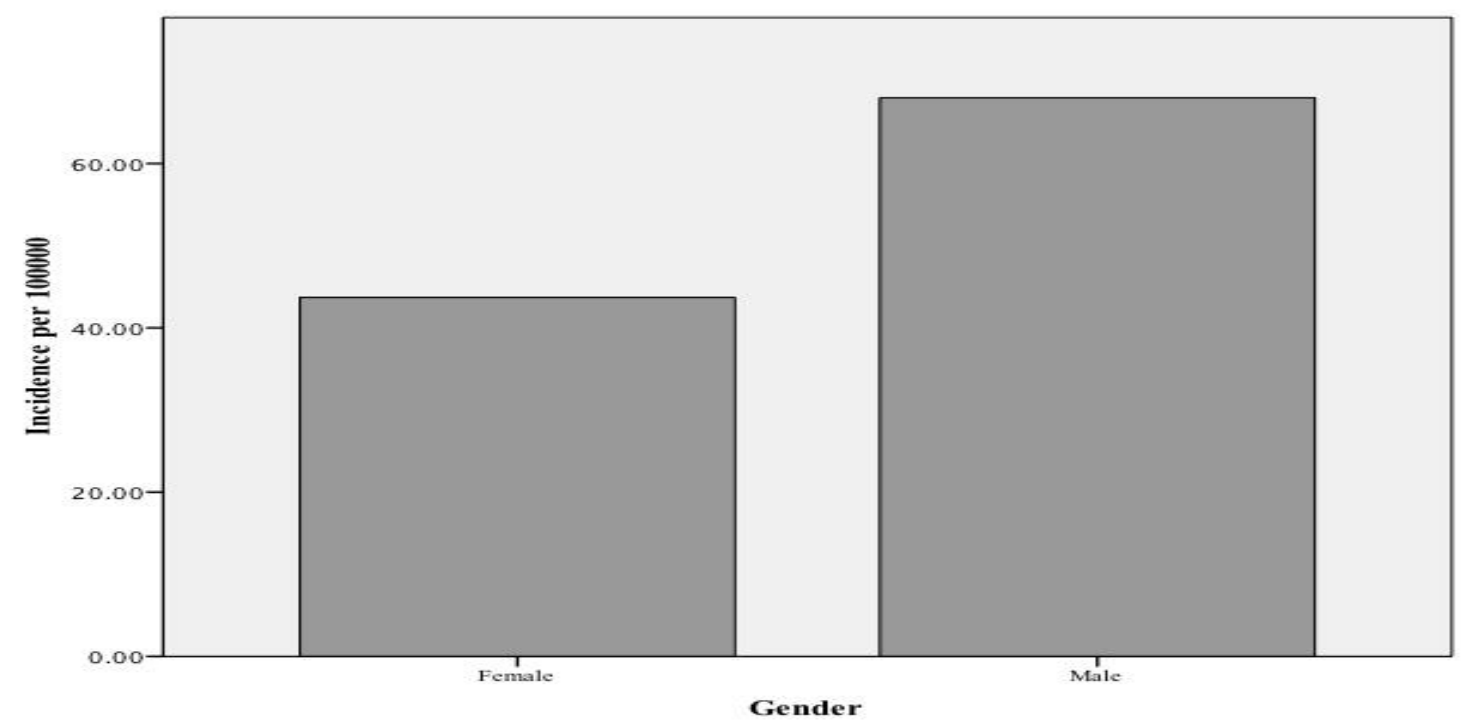

Figure 3. Incidence of IPD in the United States, 1979-2006, by gender.

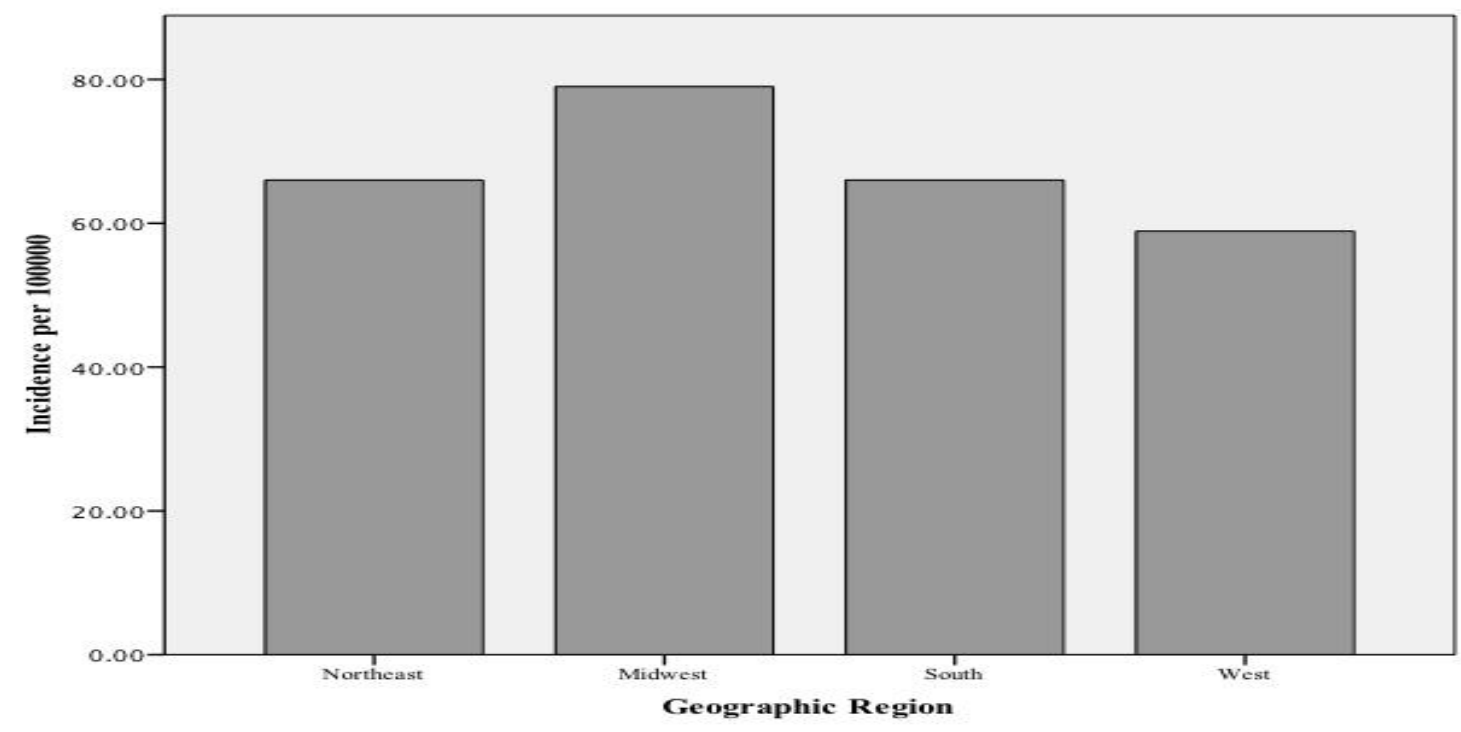

Figure 4. Incidence of IPD in the United States, 1979-2006, by geographic region. 


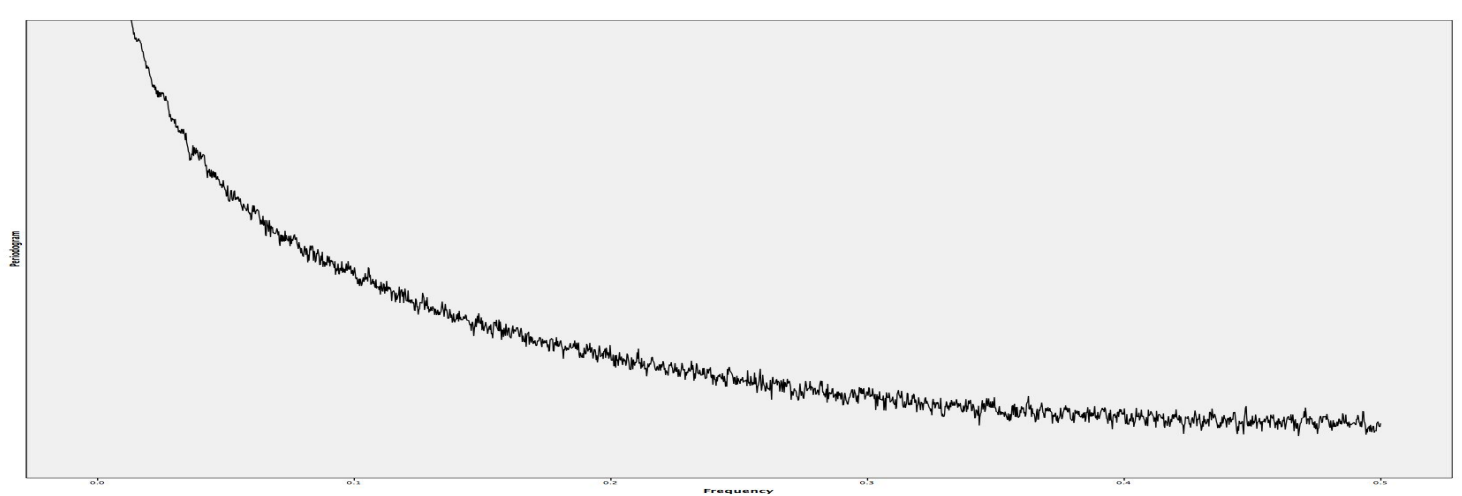

Figure 5. Periodogram of IPD incidence in the United States for the period from 19792006, inclusive of all ages, genders, and geographic regions.

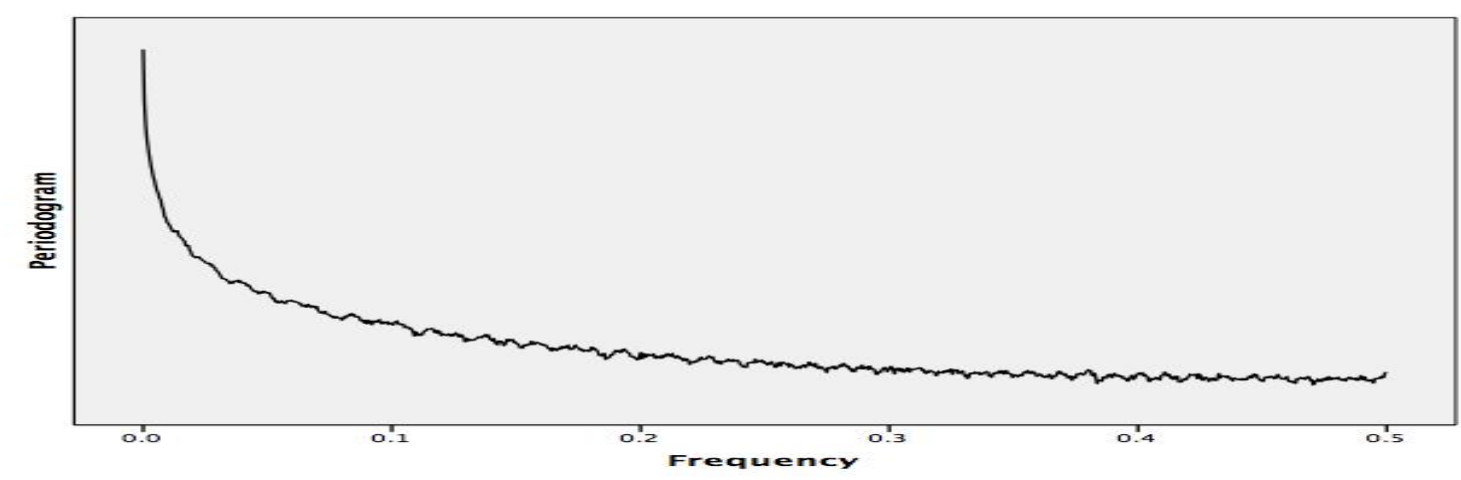

Figure 6. Periodogram of IPD incidence in the United States for the period from 19792006, by gender, male.

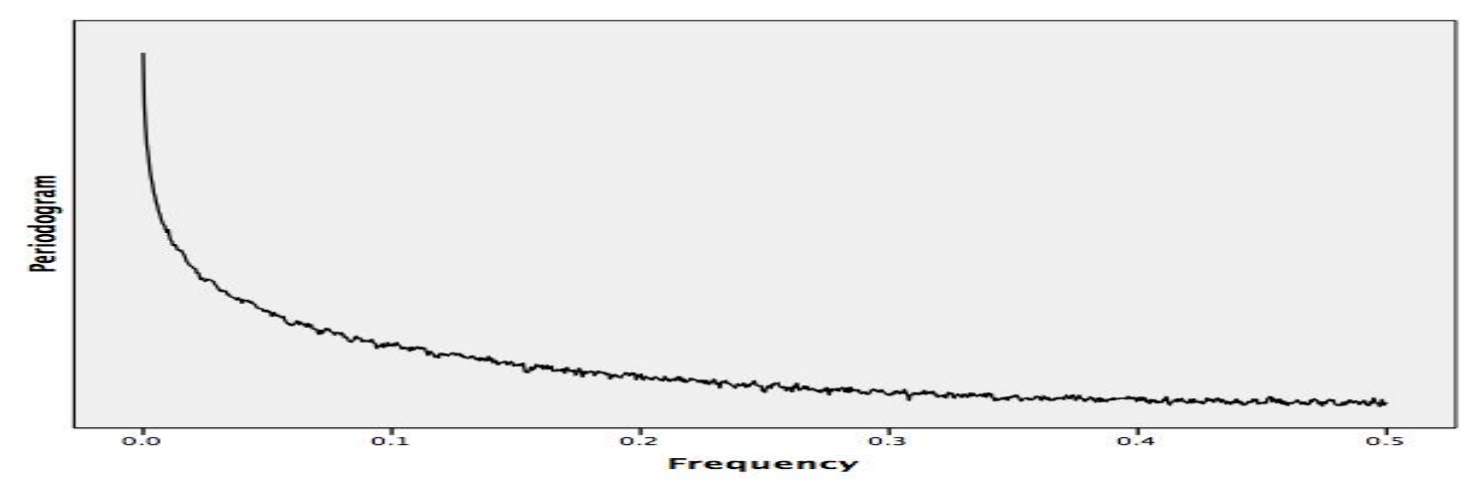

Figure 7. Periodogram of IPD incidence in the United States for the period from 19792006, by gender, female. 


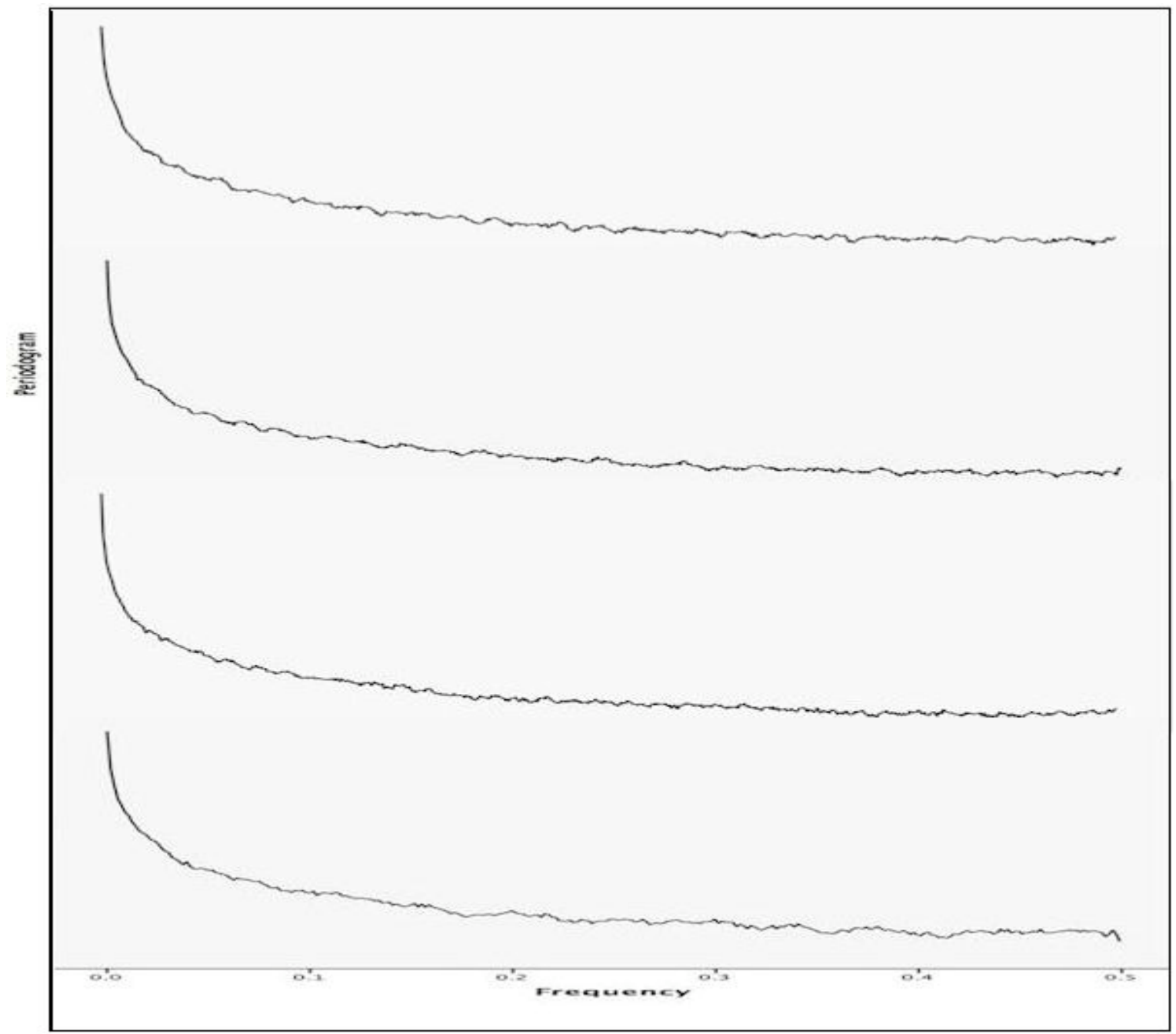

Figure 8. Periodogram of IPD incidence in the United States for the period from 19792006, Northeast, Midwest, South, and West census regions (from top to bottom). 


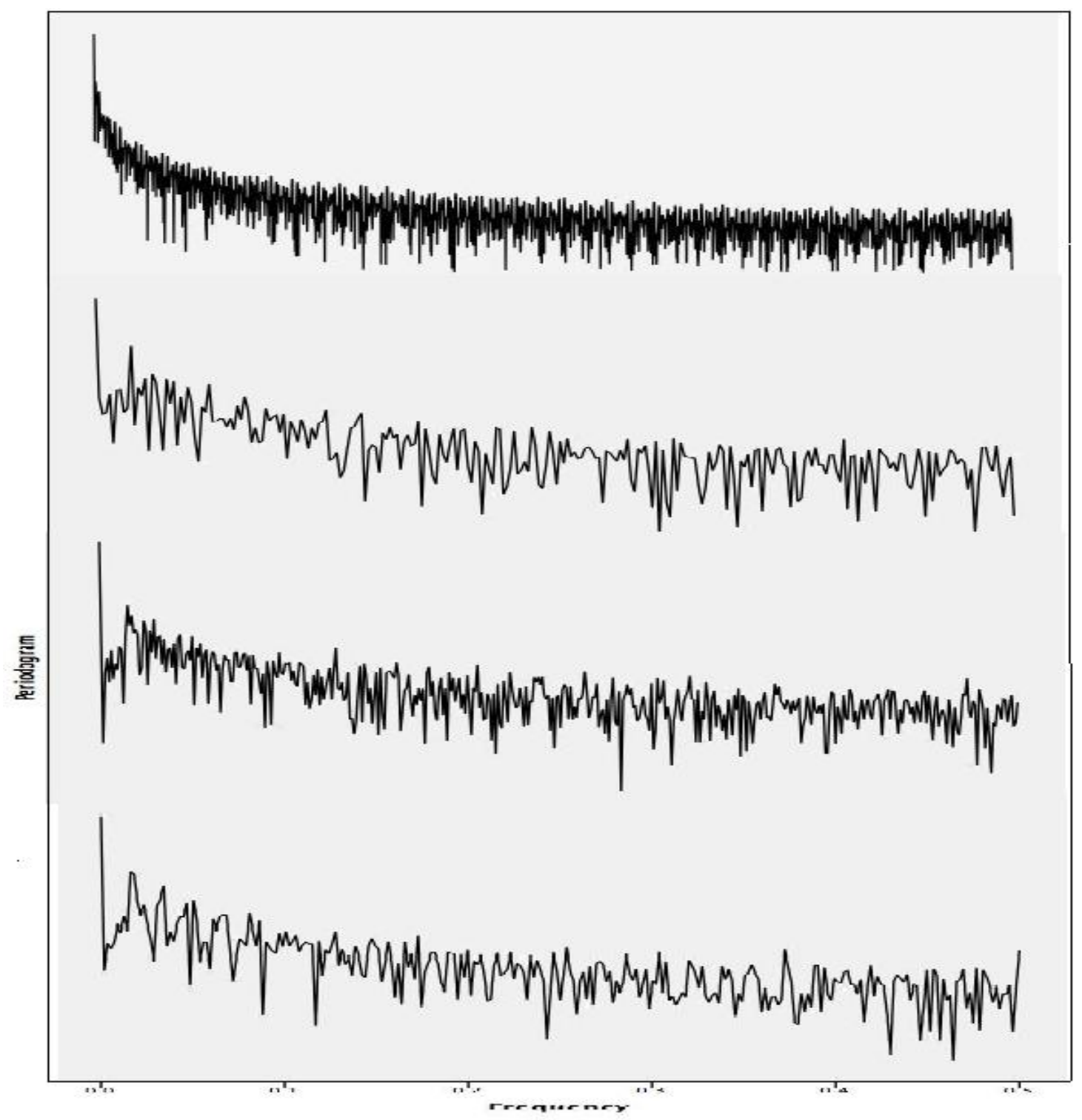

Figure 9. Periodogram of IPD incidence in the United States for the period from 19792006, 0-4 years of age, 55-64 years of age, 65-74 years of age, 75 years of age and older (from top). 


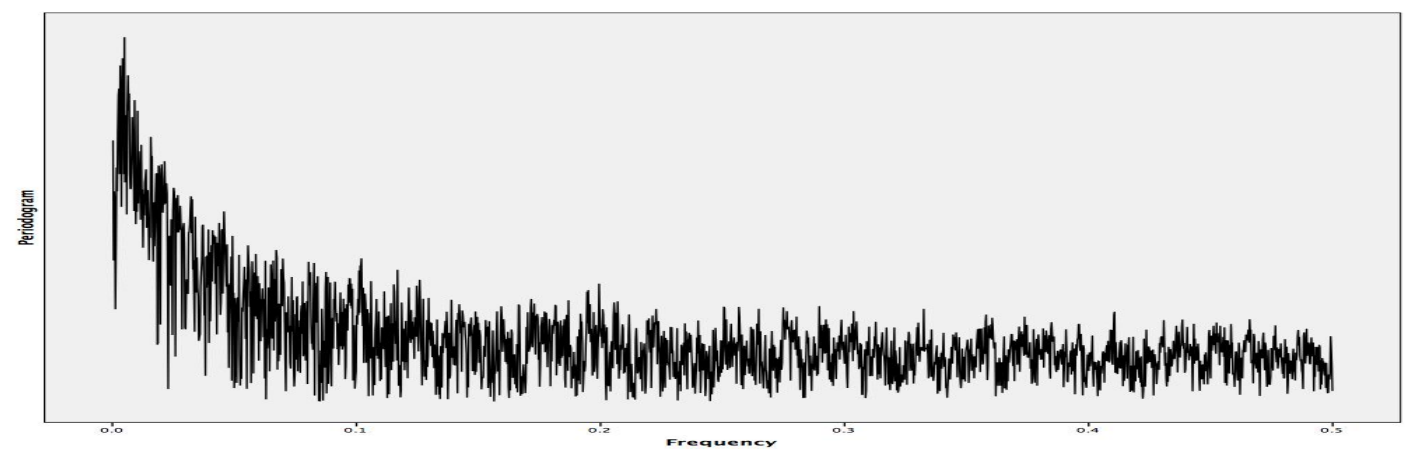

Figure 10. Periodogram of IPD incidence in the United States for the period from 19792006, analyzed for seasonality of outbreaks.

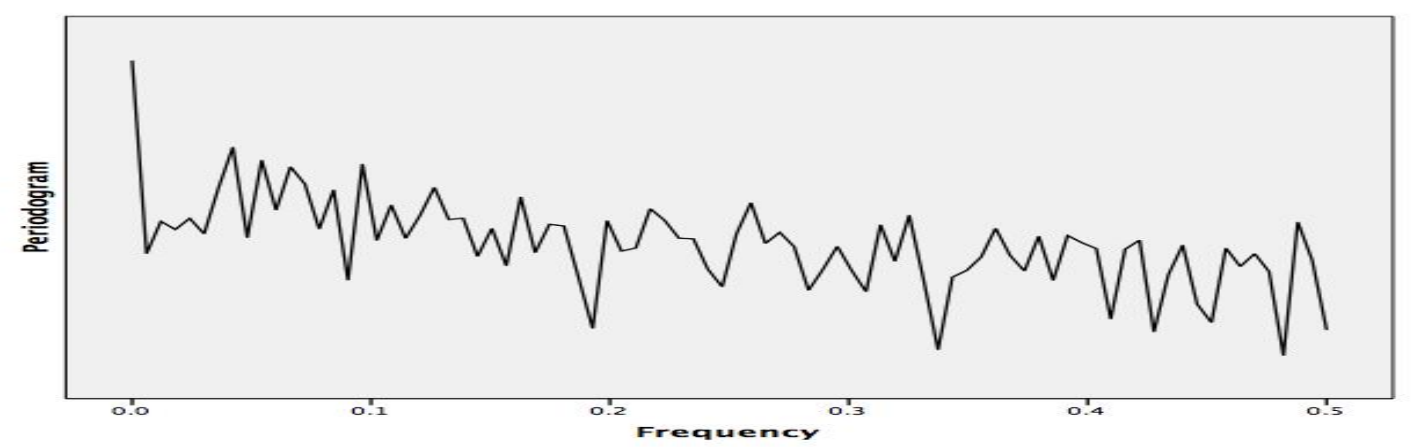

Figure 11. Periodogram of IPD incidence in the United States for the period from 19792006, 5-14 years of age.

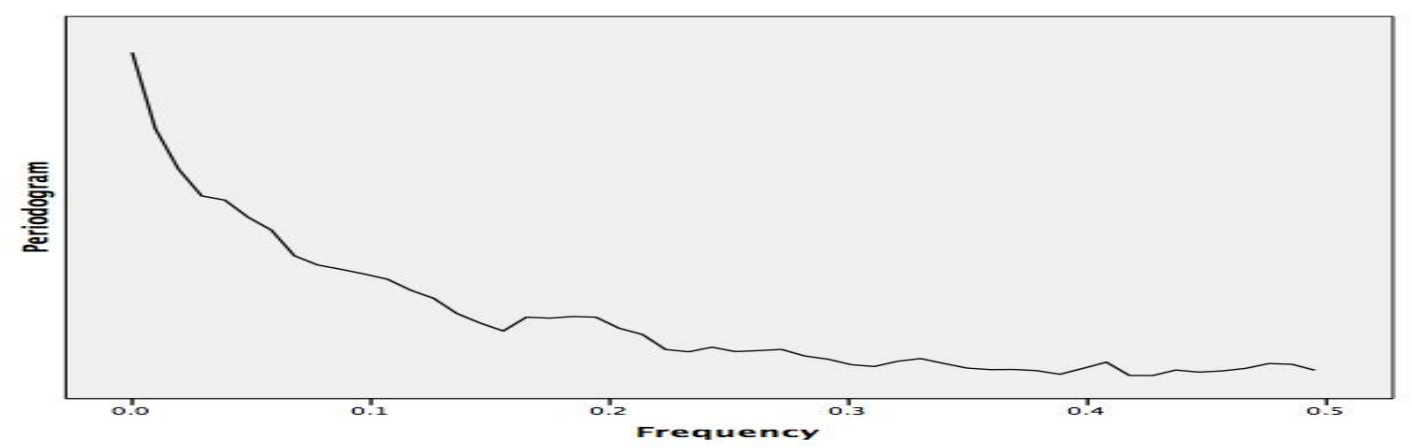

Figure 12. Periodogram of IPD incidence in the United States for the period from 19792006, 15-24 years of age. 


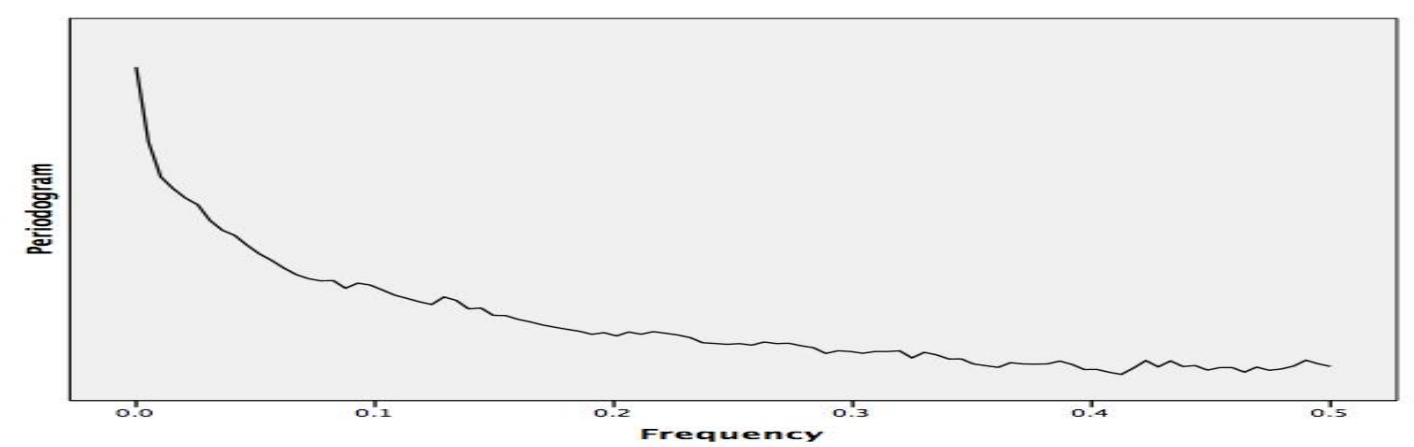

Figure 13. Periodogram of IPD incidence in the United States for the period from 19792006, 25-34 years of age.

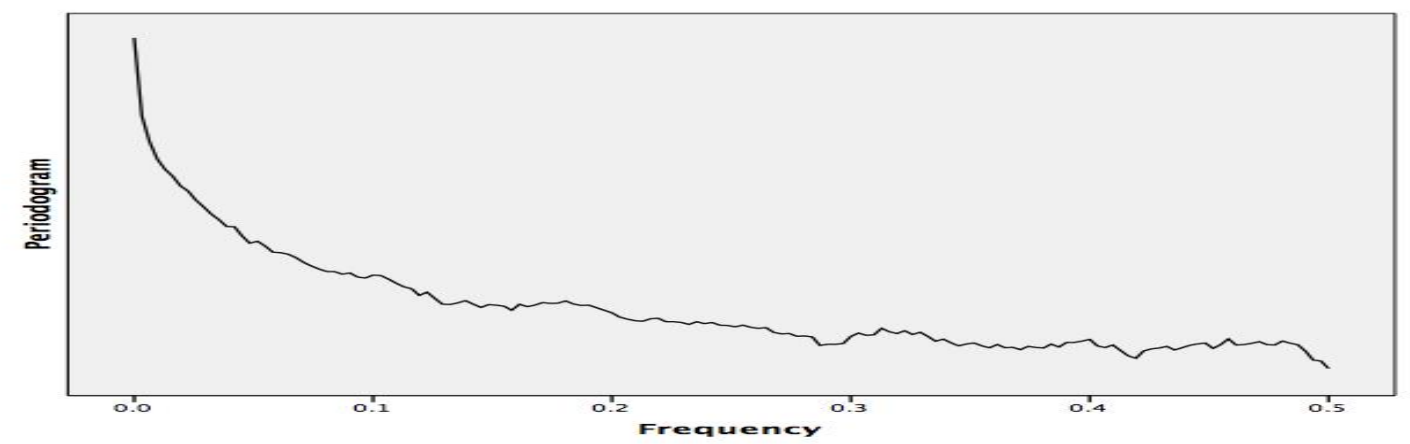

Figure 14. Periodogram of IPD incidence in the United States for the period from 19792006, 35-44 years years of age.

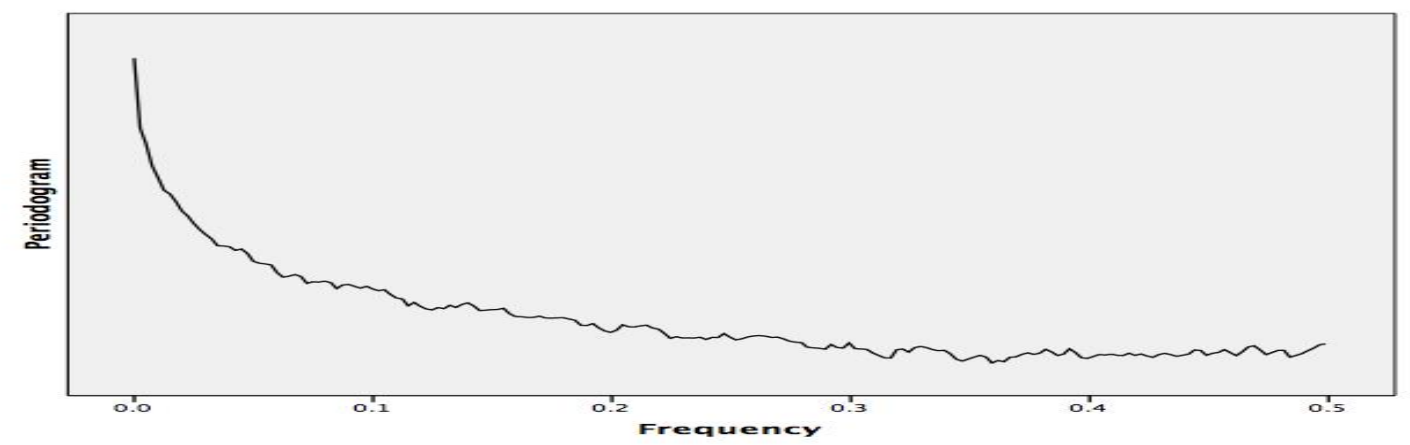

Figure 15. Periodogram of IPD incidence in the United States for the period from 19792006, 45-54 years years of age. 
Curriculum Vitae

CarlEdkrode,MPH,RRT-NPS

Professional experience
March 2003 - Present Mt Hood Community College

Program Director/Director of Clinical Education/Instructor, Respiratory Care Program

Program Director for a CoARC-accredited Advanced Respiratory Therapy Associate Degree Program, with responsibility for the planning, coordination, supervision, and evaluation of didactic and clinical instruction in Advanced Practice Respiratory Therapy. Program Advisor. Clinical and classroom instructor for Neonatal, Pediatric and Adult Respiratory General and Critical Care, including Mechanical Ventilation and Advanced Life Support, Chronic and Infectious Respiratory Diseases, Emergency Respiratory Care, Advanced Cardiopulmonary Life Support, and Principles of Pharmacology. Multidisciplinary (Nursing, Surgery Technologist, Physical Therapy Assistant programs) instructor for HIPAA, Mechanical Ventilation and Advanced Life Support, and Arterial Blood Gas Analysis and Interpretation. Responsible for recruiting, supervising, and retaining clinical instructors.

Responsible for negotiating and monitoring contracts with eight clinical sites. High Fidelity Simulation Instructor Trainer.

June 1997 - September 2006 Providence St Vincent Hospital, Portland, Oregon

Lead Respiratory Therapist

Lead Respiratory Therapist at a 510-bed tertiary care Hospital and Medical Center. Responsible for the conduct of Respiratory Therapy for Neonatal, Pediatric, and Adult patients throughout the institution, with emphasis on adult critical care; first-line supervisor for therapist staff. Clinical Preceptor and educator for Adult Emergency and Critical Care, and Pediatric Emergency Care. Night Shift Scheduler, Basic and Advanced Skills Laboratory Instructor. Practiced Advanced Cardiac Life Support, Pediatric Advanced Life Support, Neonatal Resuscitation, and Advanced Hazardous Materials Life Support. Toxic Exposure Area/Containment Facility (Chemical/Biological/Nuclear) qualified. Multidisciplinary educator for Critical Care Nurses and Internal Medicine Residents. 
October 1998 - May 1999 Oregon Health Sciences University Hospital

On-Call Respiratory Therapist

December 1994 - November 1997 United States Air Force; American Medical Response

Bitburg, Germany; Portland, Oregon

Emergency Medical Technician

Basic and Advanced Life Support in the Emergency Room and Pre-Hospital settings. Instructor/Evaluator for National Registry of Emergency Medical Technicians Basic Course, Advanced PreHospital Life Support Instructor, Advanced Pre-Hospital Burn Life Support Practitioner.

September 1976 - October 1996 United States Air Force Various Locations

Senior Non-Commissioned Officer, United States Air Force Master Armament Systems Specialist (Conventional/Special Weapons); Technical Instructor; Instructor Supervisor; Course and Technical Writer; Management Auditor/Inspector General Investigator; Department Head of Academics, Aircraft Maintenance and Weapons Branches; Crash Recovery Team Leader; Strike Operations Team Leader/Team Member; Assistant Director of Public Safety; Nuclear and Explosives Safety Officer; Assistant Director of Military Readiness; Conventional Forces in Europe and Vienna Document Treaty Compliance Officer; Emergency Room Technician. Graduate, U.S. Air Force Senior Non-Commissioned Officer Academy. Operation Desert Shield/Desert Storm, Operation Provide Comfort III.

Education

June 2006 - Present Walden University, College of Social, Behavioral and Health Sciences, School of Health and Human Services

Baltimore, Maryland

Student, $\mathrm{PhD}$ in Public Health

Specialization in Epidemiology

October 2002 - January 2005 California College for Health Sciences

Salt Lake City, Utah

Master of Public Health

Cum Laude 
October 1999 - September 2002 California College for Health Sciences

Bachelor of Science in Health Services

Emphasis in Respiratory Care

September 1996 - June 1998 Mt Hood Community College

Gresham, Oregon

Associate of Applied Science in Respiratory Care

Accreditations

Registered Respiratory Therapist-Neonatal/Pediatric Specialist

Advanced Cardiac Life Support Instructor

Basic Life Support Instructor

Additional

professional activities and publications
1999 - 2000, Adjunct Clinical Faculty, Respiratory Care Distance Education Program, Weber State University

2001 - 2005, Adjunct Clinical Faculty, Respiratory Care Distance Education Program, California College for Health Sciences

2001 - 2007, Field Medic, Northwest Rock Medicine

2002 - Author, To Lavage or Not to Lavage, That Is the Question: A Literature Review on the Practice of Saline Lavage with Artificial Airways, in Breath Sounds, the Newsletter of the Oregon Society for Respiratory Care

2003 - Present, Adjunct Faculty, Portland Community College Institute for Health Professionals

2003 - Presenter to the Oregon State Annual Conference of the Society for Critical Care Medicine, Fifth Generation Mechanical Ventilator Workshop

2004 - Reviewer for the Providence Health System Portland Service Area Severe Acute Respiratory Disease Policy and Protocol

2004 - Present, Program Developer/ Principle Investigator, In-Patient Pneumococcal Vaccination Program, Infection Control Department, Providence St. Vincent Hospital and Medical Center

2006 - Co-Author with Hicks, G., Respiratory Disease: A Case Study 
Approach to Patient Care, Chapter 9, Burn and Inhalation Injuries, and Chapter 12, Chest Trauma (ed. Wilkins et al.; F.A. Davis Publishing)

2007 - Co-author (Eckrode, C., Church, N., English, W.) Development, implementation, and assessment of a nursing assessment/standing orders-based in-patient pneumococcal vaccination program. American Journal of Infection Control

2007 - Present, Reviewer, American Journal of Infection Control

2007 - Present, Abstract Reviewer, American Public Health Association

2007- Present, Reviewer, Textbooks, Elsevier Publishing

2007 - Present, Medical Reserve Corp member, Multnomah County Public Health Department

2009 - Present, Adjunct Faculty, Respiratory Care Bachelor's Degree Completion Program, Oregon Institute of Technology

2009 - Present, Member, Mt. Hood Community College Pandemic Response Planning Committee/Incident Response Team

Professional memberships
American Public Health Association

American Association for Respiratory Care

Oregon Society for Respiratory Care 